\title{
Curvatura extrínseca de órbitas de representações
}

\author{
Artur Bicalho Saturnino
}

\author{
DisSERTAÇÃO APRESENTADA \\ AO \\ Instituto De Matemática e Estatística \\ DA \\ UNIVERSIDADE DE SÃo PAUlo \\ PARA \\ OBTENÇÃO DO TÍTULO \\ $\mathrm{DE}$ \\ Mestre em CiênCIAS
}

\author{
Programa: MAT \\ Orientador: Prof. Dr. Claudio Gorodski
}

Durante o desenvolvimento deste trabalho o autor recebeu auxílio financeiro do $\mathrm{CNPq}$

São Paulo, abril de 2017 


\section{Curvatura extrínseca de órbitas de representações}

Esta versão da dissertação contém as correções e alterações sugeridas pela Comissão Julgadora durante a defesa da versão original do trabalho, realizada em 25/05/2017. Uma cópia da versão original está disponível no

Instituto de Matemática e Estatística da Universidade de São Paulo.

Comissão Julgadora:

- Prof. Dr. Claudio Gorodski (orientador) - IME-USP

- Prof. Dr. Luiz San Martin - UNICAMP

- Pós Doc. Fancisco Gozzi - IME-USP 


\section{Agradecimentos}

Agradeço ao meu orientador, Claudio, pela paciência, pelas inúmeras instruções e pela disponibilidade. Agradeço aos meus pais pelo apoio e pelo incentivo. Sem eles não seria possível completar esta dissertação. 


\section{Resumo}

\section{SATURNINO, A. B. Curvatura extrínseca de órbitas de representações.}

2017. Dissertação (Mestrado) - Instituto de Matemática e Estatística, Universidade de São Paulo, São Paulo, 2017.

Seja $K$ um grupo de Lie compacto agindo na esfera unitária $S^{n}$ por isometrias. Mostramos como uma cota superior para as curvaturas principais de uma órbita dessa ação pode ser usada (mas não é suficiente) para encontrar uma cota inferior para o diâmetro do espaço de órbitas $S^{n} / K$. Em seguida mostramos que existe uma órbita $K p$ com curvaturas principais majoradas por $4 \sqrt{14}$.

Palavras-chave: Grupos de Lie, Representações Ortogonais, Ações Isométricas, Curvatura Extrínseca 


\section{Abstract}

\section{SATURNINO, A. B. Extrinsic curvature of orbits of representations.}

2017. Dissertação (Mestrado) - Instituto de Matemática e Estatística, Universidade de São Paulo, São Paulo, 2010.

Let $K$ be a compact Lie group acting on the unit sphere $S^{n}$ by isometries. We show how an upper bound on the principal curvatures of one orbit can be used (but is not sufficient) to obtain a lower bound for the diameter of the orbit space $S^{n} / K$. Then we show that there is an orbit $K p$ with principal curvatures bounded from above by $4 \sqrt{14}$.

Keywords: Lie Groups, Orthogonal Representations, Isometric Actions, Extrinsic Curvature 


\section{Introdução}

Seja $K$ um grupo de Lie compacto agindo por isometrias na esfera unitária $S^{n}$. O espaço de órbitas $S^{n} / K$ tem uma métrica natural dada pela distância riemanniana entre as órbitas. Karsten Grove levantou a seguinte questão:

\section{Quão pequeno pode ser o diâmetro de $S^{n} / K$ ?}

Claramente o diâmetro de $S^{n} / K$ é zero se a ação de $K$ for transitiva, portanto assumimos que a ação não é transitiva. O diâmetro de $S^{1} / K$ pode ser arbitrariamente pequeno, bastando tomar $K=\mathbb{Z}_{n}$ para $n$ grande o suficiente. Foi mostrado em [Gre00] que para todo $n \geq 2$ existe uma cota inferior, dependendo de $n$, para $\operatorname{diam}\left(S^{n} / K\right)$. Porém essa cota vai a zero com $n$ tendendo ao infinito. Em [McG93, MS05, Gre00] e [DGMS09] foi mostrado que, para alguns tipos de ação, $\operatorname{diam}\left(S^{n} / K\right)$ tem uma cota inferior independente de $n$. Em [GL16] é mostrado que para ações de co-homogeneidade maior que um existe uma cota superior, independente de $n$, para o ínfimo das curvaturas de $S^{n} / K$. Pelo Teorema de Bonnet-Myers essa é uma condição necessária para a existência de uma cota inferior independente de $n$ para o diâmetro desses espaços. Esses fatos tornam plausível a existência de um $\epsilon>0$ tal que $\operatorname{diam}\left(S^{n} / K\right) \geq \epsilon$ sempre que $n \geq 2$, mas ainda não se sabe se tal $\epsilon$ existe.

Aqui não trataremos diretamente do diâmetro do quociente de esferas. Observaremos que geodésicas não podem realizar a distância entre órbitas além de um ponto focal e que, se as curvaturas principais de uma órbita são majoradas por $C$, então pontos focais dessa órbita só ocorrem em geodésicas de comprimento pelo o menos $\operatorname{arccot}(C)$. Esse fato não é suficiente para obter uma cota inferior para o diâmetro do espaço quociente, mas veremos como esse fato, juntamente com informações sobre as intersecções entre geodésicas perpendiculares a essa órbita, pode ser usado para obter tal cota. Essa relação entre o diâmetro do espaço de órbitas e as curvaturas principais de uma órbita motiva os resultados principais desse texto. Resumidamente, esses resultados implicam que toda ação isométrica de um grupo de Lie compacto em uma esfera tem uma órbita onde as curvaturas principais são majoradas por $4 \sqrt{14}$.

Como toda ação isométrica na esfera $S^{n}$ se estende para uma representação ortogonal em $\mathbb{R}^{n+1}$, nós dizemos que uma ação isométrica em $S^{n}$ é redutível se a representação associada é redutível. É fácil ver que o diâmetro do espaço de órbitas de ações redutíveis é $\pi$ se essa ação tem uma direção fixa, e $\frac{\pi}{2}$ caso contrário. Então, do ponto de vista do diâmetro, ações redutíveis não são interessantes. Porém as órbitas de algumas ações redutíveis possuem propriedades interessantes do ponto de vista de suas curvaturas extrínsecas. Considere a ação de $S^{1}$ em $S^{2}$ por rotações em torno de um eixo. As órbitas dessa ação são os paralelos e o polos norte e sul. Como o equador é um grande círculo, as suas curvaturas principais são zero. Por outro lado, é fácil mostrar que as curvaturas principais dos paralelos tentem ao infinito quando esses se aproximam dos polos. Qualquer ação isométrica de um 
toro em uma esfera possui uma órbita que é um grande círculo, bastando tomar a intersecção da esfera com um subespaço irredutível de dimensão dois. Nem sempre existem órbitas com curvaturas principais arbitrariamente grandes (todas as órbitas da ação de Hopf $S^{1} \times S^{2 n+1} \rightarrow S^{2 n+1}$ são grandes círculos), mas se uma ação isométrica em uma esfera fixa algum ponto e tem órbitas de dimensão positiva, então essa ação tem órbitas com curvaturas principais arbitrariamente grandes.

Essa dissertação é divida em dois capítulos. O Capítulo 1 motiva e fundamenta os principais resultados desse texto. Na Secção 1.1 apresentamos algumas definições básicas, e na Secção 1.2 mostramos a relação entre a curvatura de uma órbita e o diâmetro do quociente que foi apresentada acima. Na Secção 1.3 classificamos as representações irredutíveis de grupos de Lie compactos nos tipos real, complexo e quaterniônico e mostramos alguns resultados básicos sobre a segunda forma fundamental das órbitas de representações. Na Secção 1.4 apresentamos alguns fatos sobre a estrutura das álgebras de Lie compactas, suas complexificações e representações.

O Capítulo 2 contêm os principais resultados deste texto. Nele mostramos a existência de uma órbita onde as curvaturas principais não excedem $4 \sqrt{14}$. Mais especificamente, no Capítulo 2 apresentamos e provamos os Teoremas 2.1.1 e 2.1.2 bem como algumas de suas consequências. Simplificando, o Teorema 2.1.1 nos diz que se uma ação isométrica de um grupo de Lie compacto em uma esfera se estende para uma representação ortogonal de tipo complexo ou quaterniônico, então as curvaturas principais da órbita de um vetor de peso maximal são majoradas por $4 \sqrt{7}$. Já o Teorema 2.1.2 nos permite usar o Teorema 2.1.1 para obter órbitas com curvaturas principais majoradas por $4 \sqrt{14}$ em ações que se estendem a representações ortogonais de tipo real. 


\section{Sumário}

1 Preliminares $\quad 1$

1.1 Ações isométricas de grupos de Lie compactos . . . . . . . . . . . . . . . . . . . . 1

1.2 Curvatura extrínseca e distância entre órbitas . . . . . . . . . . . . . . 2

1.2.1 Geodésicas realizando a distância entre uma subvariedade e um ponto . . . . 2

1.2.2 Distância focal de órbitas de representações . . . . . . . . . . . . . . . . 5

1.3 Representações de grupos de Lie compactos . . . . . . . . . . . . . . . . . 7

1.3.1 Classificação nos tipos real, complexo e quaterniônico . . . . . . . . . . . . 8

1.3.2 Segunda forma fundamental das órbitas de uma representação . . . . . . . . . 10

1.4 Álgebras de Lie compactas e suas representações . . . . . . . . . . . . . . . . . . 11

1.4.1 Álgebras de Lie compactas e suas complexificações . . . . . . . . . . . . . . . 11

1.4.2 Representações de álgebras de Lie compactas . . . . . . . . . . . . . . . . . 13

2 Resultados $\quad 17$

2.1 Apresentação dos resultados . . . . . . . . . . . . . . . . . . . . 17

2.2 Curvatura de órbitas de representações unitárias . . . . . . . . . . . . . . 18

2.2 .1 Estimativas para $\mathfrak{k}$ clássica . . . . . . . . . . . . . . . 23

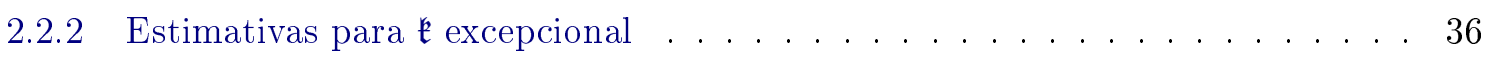

2.3 Curvatura de órbitas de representações de tipo real . . . . . . . . . . . . . . 36

2.3.1 Demonstração do Lema $2.3 .1 \ldots \ldots$. . . . . . . . . . . . . . . 37

$\begin{array}{ll}\text { A Dados sobre sistemas de raízes reduzidos } & 41\end{array}$

$\begin{array}{ll}\text { B Código } & 43\end{array}$

Referências Bibliográficas $\quad 45$ 


\section{Capítulo 1}

\section{Preliminares}

Nesse capítulo motivamos os resultados do Capítulo 2 e apresentamos ferramentas que serão usadas para provar esses resultados. Começamos na Secção 1.1 apresentando conceitos e resultados básicos relativos a ações isométricas de grupos de Lie Compactos em uma variedade Riemanniana qualquer. A Seç̧ão 1.2 tem como objetivo demonstrar o Corolário 1.2.5, que mostra como uma cota superior para as curvaturas principais de uma órbita de uma ação isométrica em uma esfera pode ser utilizada (mas não é suficiente) para determinar uma cota inferior para diâmetro do espaço de órbitas. As Secções 1.3 e 1.4 apresentam alguns resultados algébricos e geométricos referentes às representações de grupos de Lie compactos que serão usados no Capítulo 2.

\subsection{Ações isométricas de grupos de Lie compactos}

Uma ação isométrica de um grupo de Lie $K$ em uma variedade Riemanniana $M$ é uma aplicação suave $\Theta: K \times M \rightarrow M$ tal que:

(i) $\Theta\left(k_{1}, \Theta\left(k_{2}, p\right)\right)=\Theta\left(k_{1} k_{2}, p\right)$ para todo $k_{1}, k_{2} \in K$ e $p \in M$;

(ii) $\Theta(e, p)=p$ para todo $p \in M$, onde $e$ é o elemento neutro de $K$;

(iii) as aplicações $\Theta(k, \cdot): p \mapsto \Theta(k, p)$ são isometrias para todo $k \in K$.

Usaremos a notação $k \cdot p=\Theta(k, p)$.

A órbita de $\Theta$ por $p \in M$ é o conjunto

$$
K p=\{k \cdot p: k \in K\} .
$$

Sempre assumiremos que $K$ é compacto. Da Proposição 3.41 em [AB15] temos que órbitas de ações isométricas por grupos compactos são subvariedades mergulhadas e

$$
T_{p}(K p)=\left\{\left.\frac{d}{d s} \operatorname{Exp}(s x) \cdot p\right|_{s=0}: x \in \mathfrak{k}\right\}
$$

onde $\mathfrak{k}$ é a álgebra de Lie do grupo $K$ e Exp: $\mathfrak{k} \rightarrow K$ é a aplicação exponencial.

O espaço de órbitas da ação é o espaço métrico formado pelo conjunto

$$
M / K=\{K p: p \in M\},
$$

com métrica dada por

$$
d\left(K p_{1}, K p_{2}\right)=\inf \left\{d\left(k_{1} \cdot p_{1}, k_{2} \cdot p_{2}\right): k_{1}, k_{2} \in K\right\} \forall p_{1}, p_{2} \in M,
$$

onde $d$ é a distância em $M$ definida pela métrica riemanniana. 


\subsection{Curvatura extrínseca e distância entre órbitas}

Seja $S^{n}$ uma esfera unitária e $K$ um grupo de Lie compacto agindo em $S^{n}$ por isometrias. O objetivo dessa secção é mostrar como uma cota superior para as curvaturas principais de uma órbita $K p$ nos dá informação parcial sobre as geodésicas que realizam a distância entre $K p$ e outras órbitas. Como para todo $q \in S^{n}$ temos que $d(K p, K q)=d(K p, q)$, faz sentido estudar as geodésicas que realizam a distância entre $K p$ e pontos de $S^{n} \backslash K p$.

Começamos pela Subsecção 1.2.1 onde, de maneira mais geral, consideramos $(M, g)$ uma variedade riemanniana completa, $N \subset M$ uma subvariedade fechada e estudando geodésicas que realizam a distância entre $N$ e pontos de $M \backslash N$ para provar a Proposição 1.2.2, que caracteriza o ponto além do qual uma geodésica que parte perpendicularmente de $N$ não realiza a distância a partir de $N$. Na Subsecção 1.2.1 aplicamos essa proposição ao caso onde $M=S^{n}$ e $N=K p$ para provar o Corolário 1.2.5, que motiva os principais resultados do Capítulo 2.

\subsubsection{Geodésicas realizando a distância entre uma subvariedade e um ponto}

O objetivo dessa subsecção é mostrar a Proposição 1.2.2. Vários dos resultados, definições e provas dessa secção são adaptados do capítulo III.2 de [Sak96]. Note que a convenção para o operador de Weingarten usada nessa referência é diferente da usada aqui. Nessa Subsecção $(M, g)$ é uma variedade riemanniana completa com conexão Levi-Civita $\nabla$, por abuso de notação também usamos $\nabla$ para as conexões definidas pelo pull-back de aplicação suaves em $M$. Usamos $\mathcal{R}$ para a curvatura de $M . N \subset M$ é uma subvariedade fechada de $M, \nu N$ é o fibrado normal de $N$ e $(\cdot)^{\perp}$ é a projeção ortogonal em $\nu N$. Escrevemos $T^{1} N$ (resp. $\nu^{1} N$ ) para o fibrado dos vetores tangentes (resp. normais) a $N$ com norma um.

A segunda forma fundamental de $N$ em $p$ é a aplicação bilinear

$$
\begin{aligned}
\mathrm{II}_{p}: T_{p} N \times T_{p} N & \rightarrow \nu_{p} N \\
(x, y) & \mapsto\left(\nabla_{X} Y\right)_{p}^{\perp}
\end{aligned}
$$

onde $X$ e $Y$ são campos suaves em $M$ que se restringem a campos suaves de $N$ tais que $X_{p}=x$ e $Y_{p}=y$. Note que a segunda forma fundamental está bem definida e é simétrica.

O operador de Weingarten de $N$ na direção $\xi \in \nu_{p} N$ é a aplicação linear $A_{\xi}: T_{p} N \rightarrow T_{p} N$ definida por

$$
g\left(A_{\xi}(x), y\right)=g\left(\mathrm{II}_{p}(x, y), \xi\right) \forall x, y \in T_{p} N .
$$

Segue que $A_{\xi}$ é simétrica, e portanto diagonalizável. As curvaturas principais de $N$ na direção $\xi$ são os autovalores de $A_{\xi}$ e as curvaturas principais de $N$ em $p$ são todos autovalores de todos $A_{\xi}$ $\operatorname{com} \xi \in \nu_{p}^{1} N$.

Dizemos que uma geodésica $\gamma:[0, l] \rightarrow M$ realiza a distancia entre $N$ e um ponto $q \in M \backslash N$ se

$$
\gamma(0) \in N, \gamma(l)=q \text { e } L(\gamma)=d(N, q)=\inf _{p \in N} d(p, q)
$$

onde $L(\gamma)$ é o comprimento de $\gamma$. Como $N$ é fechada e $M$ é completa, temos que para todo $q \in M \backslash N$ existe uma geodésica realizando a distância de $N$ a $q$. Usando a primeira variação do comprimento é fácil mostrar que se uma geodésica $\gamma[0, l] \rightarrow M$ realiza a distância de $N$ até qualquer ponto de $M \backslash N$, então $\gamma$ é uma $N$-geodésica, isso é $\gamma^{\prime}(0) \in \nu N$.

Um campo suave $J$ ao longo de uma geodésica $\gamma$ é um campo de Jacobi se

$$
-J^{\prime \prime}(s)+\mathcal{R}\left(\gamma^{\prime}(s), J(s)\right) \gamma^{\prime}(s)=0 \forall s \in[0, l],
$$

onde $J^{\prime \prime}(s)=\nabla_{\frac{\partial}{\partial s}} \nabla_{\frac{\partial}{\partial s}} J(s)$. Uma variação por geodésicas de uma geodésica $\gamma$ é uma variação suave $(t, s) \mapsto \gamma_{t}(s)$ de $\gamma$ tal que para todo $t \in(-\epsilon, \epsilon)$ a curva $s \mapsto \gamma_{t}(s)$ é uma geodésica. O campo variacional induzido por uma variação por geodésicas é um campo de Jacobi e todo campo de Jacobi é induzido por uma variação por geodésicas. Particularmente, os campos de Jacobi ao longo 
de uma $N$-geodésica induzidos por variações por $N$-geodésicas são chamados $N$-campos de Jacobi. Seja $J$ um campo de Jacobi ao longo da $N$-geodésica $\gamma:[0, l] \rightarrow M$ com velocidade inicial $\gamma^{\prime}(0)=\xi$. Então exite uma variação por $N$-geodésicas $(t, s) \mapsto \gamma_{t}(s)$ induzindo $J$ se, e somente se, as condições iniciais

$$
J(0) \in T N \quad \text { e } \quad J^{\prime}(0)+A_{\xi}(J(0)) \in \nu N
$$

forem satisfeitas. Essas condições vêm dos fatos que $\gamma_{t}(0) \in N$ e $\gamma_{t}^{\prime}(0) \in \nu N$ para todo $t \in(-\epsilon, \epsilon)$, respectivamente.

Um ponto $\gamma(l)$ é chamado ponto focal de $N$ ao longo de $\gamma$ se existir um $N$-campo de Jacobi não nulo $J$ ao longo de $\gamma$ tal que $J(l)=0$. Pontos focais são uma generalização de pontos conjugados, e a Proposição 1.2.1 generaliza o fato que uma geodésica $\gamma:[0, l] \rightarrow M$ não pode realizar a distância de $\gamma(0)$ a $\gamma(l)$ se houver algum ponto conjugado a $\gamma(0)$ por $\gamma$ em $\gamma([0, l))$. Antes de mostrar a Proposição 1.2.1 precisamos de introduzir alguns conceitos.

A energia de uma curva suave por partes $c:[a, b] \rightarrow M$ é definida por

$$
E(c)=\frac{1}{2} \int_{a}^{b}\left\|c^{\prime}(s)\right\|^{2} d s .
$$

Segue da desigualdade de Cauchy-Schwartz que

$$
L(c)^{2} \leq 2(b-a) E(c),
$$

onde a igualdade ocorre se, e somente se, $\left\|c^{\prime}(s)\right\|$ for constante. Seja $\gamma:[0, l] \rightarrow M$ uma $N$-geodésica, $\xi=\gamma^{\prime}(0)$ e $(s, t) \mapsto \gamma_{t}(s)$ uma variação suave por partes de $\gamma$ tal que $\gamma_{t}(0) \in N$ e $\gamma_{t}(l)=\gamma(l)$ para todo $t \in(-\epsilon, \epsilon)$. Como $\gamma$ é uma $N$-geodésica, a primeira variação da energia de $\gamma_{t}$ em $t=0$ é zero, e a segunda variação da energia em $t=0$ é:

$$
\left.\frac{d^{2}}{d t^{2}} E\left(\gamma_{t}\right)\right|_{t=0}=-g\left(A_{\xi}(X), X\right)_{0}+\int_{0}^{l}\left\|X^{\prime}\right\|^{2}+g\left(\mathcal{R}\left(\gamma^{\prime}, X\right) \gamma^{\prime}, X\right) d s,
$$

onde $X$ é o campo variacional de $\gamma_{t}$. Note que todo campo $X$ induzido por uma variação como descrita acima é tal que

$$
X(0) \in T N \quad \text { e } \quad X(l)=0 .
$$

Seja $\mathcal{V}$ o espaço dos campos suaves por partes ao longo de $\gamma$ satisfazendo as condições acima. Como a fórmula da segunda variação da energia define uma forma quadrática em $\mathcal{V}$, essa fórmula induz uma forma simétrica bilinear em $\mathcal{V}$, chamada forma do índice, que será denotada por $I_{N}$. Temos que

$$
I_{N}(X, Y)=-g\left(A_{\xi}(X), Y\right)_{0}+\int_{0}^{l} g\left(X^{\prime}, Y^{\prime}\right)+g\left(\mathcal{R}\left(\gamma^{\prime}, X\right) \gamma^{\prime}, Y\right) d s
$$

Seja $0=s_{0}<s_{1}<\cdots<s_{n}=l$ uma subdivisão de $[0, l]$ tal que $X$ e $Y$ são suaves em $\left[s_{i}, s_{i+1}\right]$ para $i=0, \cdots, n-1$. Como, em cada um desses intervalos, $g\left(X^{\prime}, Y^{\prime}\right)=g\left(X^{\prime}, Y\right)^{\prime}-g\left(X^{\prime \prime}, Y\right)$, temos que

$$
I_{N}(X, Y)=-g\left(A_{\xi}(X), Y\right)_{0}+\sum_{i=0}^{n} g\left(X^{\prime}\left(s_{i}^{-}\right)-X^{\prime}\left(s_{i}^{+}\right), Y\left(s_{i}\right)\right)+\int_{0}^{l} g\left(-X^{\prime \prime}+\mathcal{R}\left(\gamma^{\prime}, X\right) \gamma^{\prime}, Y\right) d s,
$$

onde $X^{\prime}\left(s_{i}^{-}\right)$(resp. $\left.X^{\prime}\left(s_{i+1}^{+}\right)\right)$é a derivada de $X$ em $s_{i}$ pela esquerda (resp. direita).

Proposição 1.2.1. Seja $N$ uma subvariedade de $M$ e $\gamma:[0, l] \rightarrow M$ uma $N$-geodésica. Suponha que existe $s_{1} \in(0, l)$ tal que $\gamma\left(s_{1}\right)$ é ponto focal de $N$ ao longo de $\gamma$. Então $\gamma$ não realiza a distância de $N$ a $\gamma(l)$.

Demonstração. Seja $J$ um $N$-campo de Jacobi não nulo ao longo de $\left.\gamma\right|_{\left[0, s_{1}\right]}$ tal que $J\left(s_{1}\right)=0$. Seja $Z$ o campo ao longo de $\gamma$ obtido por transporte paralelo de $-J^{\prime}\left(s_{1}\right)$ e seja $\theta:[0, l] \rightarrow \mathbb{R}$ uma aplicação suave com suporte em um intervalo $\left(s_{1}-\delta, s_{1}+\delta\right)$ onde $\delta<\min \left\{s_{1}, l-s_{1}\right\}$ tal que $\theta\left(s_{1}\right)=1$. 
Definimos o campo $\bar{Z}$ por $\bar{Z}(s)=\theta(s) Z(s)$ e campo $\bar{J}$ por $\bar{J}(s)=J(s)$ se $0 \leq s \leq s_{1}$ e $\bar{J}(s)=0$ se $s_{1} \leq s \leq l$.

Para $\alpha>0$ definimos o campo $X_{\alpha}$ por $X_{\alpha}(s)=\bar{J}(s)+\alpha \bar{Z}(s)$. Note que $X_{\alpha} \in \mathcal{V}$, então pelo Teorema III.2.1 em [Sak96] existe uma variação suave por partes $(s, t) \mapsto \gamma_{t}(s)$ de $\gamma$ induzindo $X_{\alpha}$ tal que $\gamma_{t}(0) \in N$ e $\gamma_{t}(l)=\gamma(l)$ para todo $t \in(-\epsilon, \epsilon)$. Então temos que

$$
\left.\frac{d^{2}}{d t^{2}} E\left(\gamma_{t}\right)\right|_{t=0}=I_{N}\left(X_{\alpha}, X_{\alpha}\right)=I_{N}(\bar{J}, \bar{J})+2 \alpha I_{N}(\bar{J}, \bar{Z})+\alpha^{2} I_{N}(\bar{Z}, \bar{Z}) .
$$

Vamos estudar a identidade acima vendo ela como uma função polinomial em $\alpha$. Queremos mostrar que $\left.\frac{d^{2}}{d t^{2}} E\left(\gamma_{t}\right)\right|_{t=0}<0$ para $\alpha$ suficientemente pequeno. Note que como $\left.\bar{J}\right|_{\left[s_{1}, l\right]}=0$ temos que

$$
I_{N}(\bar{J}, \bar{J})=-g\left(A_{\xi}(J), J\right)_{0}-g\left(J^{\prime}, J\right)_{0}+\int_{0}^{s_{1}} g\left(-J^{\prime \prime}+\mathcal{R}\left(\gamma^{\prime}, J\right) \gamma^{\prime}, J\right) d s .
$$

Usando que $J$ é um $N$-campo de Jacobi, concluímos que $I_{N}(\bar{J}, \bar{J})=0$. Usando esses mesmos fatos sobre $\bar{J}$ temos que

$I_{N}(\bar{J}, \bar{Z})=-g\left(A_{\xi}(J), \bar{Z}\right)_{0}-g\left(J^{\prime}, \bar{Z}\right)_{0}+g\left(J^{\prime}, \bar{Z}\right)_{s_{1}}+\int_{0}^{s_{1}} g\left(-J^{\prime \prime}+\mathcal{R}\left(\gamma^{\prime}, J\right) \gamma^{\prime}, \bar{Z}\right) d s=g\left(J^{\prime}, \bar{Z}\right)_{s_{1}}$.

Então, como $\bar{Z}\left(s_{1}\right)=-J\left(s_{1}\right)$, temos $I_{N}(\bar{J}, \bar{Z})=-\left\|J^{\prime}\left(s_{1}\right)\right\|^{2}$. Como $J$ é não nulo, devemos ter que $\left\|J^{\prime}\left(s_{1}\right)\right\|>0$. Segue que $\left.\frac{d^{2}}{d t^{2}} E\left(\gamma_{t}\right)\right|_{t=0}=-2 \alpha\left\|J^{\prime}\left(s_{1}\right)\right\|+\alpha^{2} I_{N}(\bar{Z}, \bar{Z})$. Logo $\left.\frac{d^{2}}{d t^{2}} E\left(\gamma_{t}\right)\right|_{t=0}<0$ para $\alpha>0$ suficientemente pequeno.

Fixe um $\alpha$ positivo tal que $\left.\frac{d^{2}}{d t^{2}} E\left(\gamma_{t}\right)\right|_{t=0}<0$. Então, como a primeira variação da energia de $\gamma_{t}$ é nula, temos que $E\left(\gamma_{t_{0}}\right)<E(\gamma)$ para $t_{0}$ suficientemente pequeno, o que implica que

$$
L^{2}\left(\gamma_{t_{0}}\right) \leq 2 l E\left(\gamma_{t_{0}}\right)<2 l E(\gamma)=L^{2}(\gamma) .
$$

Então $\gamma$ não pode realizar a distância de $N$ a $\gamma(l)$.

Seja $\exp ^{\perp}$ a restrição da aplicação exponencial de $M$ a $\nu N \subset T M$ e seja $\xi \in \nu N$. Então $\exp ^{\perp}(\xi)$ é ponto focal de $N$ ao longo da $N$-geodésica $s \mapsto \exp ^{\perp}(s \xi)$ se $\xi$ for ponto critico de $\exp ^{\perp}$. Definimos $\rho_{N}: \nu N \rightarrow(0, \infty]$ como a aplicação que associa a cada vetor $\xi \in \nu N$ o maior $s>0$ até o qual a geodésica com velocidade inicial $\xi$ realiza a distância de $N$ a $\exp ^{\perp}(s \xi)$, isso é:

$$
\rho_{N}(\xi)=\sup \left\{s \in[0, \infty): d\left(N, \exp ^{\perp}(s \xi)\right)=s\|\xi\|\right\} .
$$

Proposição 1.2.2. Seja $\gamma:[0, \infty) \rightarrow M$ uma $N$-geodésica e seja $s_{0}>0$. Então $\rho_{N}\left(\gamma^{\prime}(0)\right)=s_{0}$ se, e somente se, $\left.\gamma\right|_{\left[0, s_{0}\right]}$ realizar a distância de $N$ a $\gamma\left(s_{0}\right)$ e uma das condiçôes abaixo for satisfeita:

(i) $\gamma\left(s_{0}\right)$ é ponto focal de $N$ ao longo de $\gamma$, ou

(ii) existe outra $N$-geodésica realizando a distância de $N$ a $\gamma\left(s_{0}\right)$.

Demonstração. Seja $\xi=\gamma^{\prime}(0)$ e $p=\gamma(0)$. Assuma sem perda de generalidade que $\|\xi\|=1$. Primeiramente, assuma que $\rho_{N}(\xi)=s_{0}<\infty$. Mostramos que (i) ou (ii) devem ocorrer. Escolha uma sequencia estritamente decrescente $\left\{t_{i}\right\}_{i \in \mathbb{N}}$ tendendo a $s_{0}$. Para cada $i \in \mathbb{N}$ existe uma $N$ geodésica $\gamma_{i}$ com velocidade de norma um realizando a distância de $N$ a $\gamma\left(t_{i}\right)$. Sejam $\xi_{i} \in \nu^{1} N$ a velocidade inicial de $\gamma_{i}, p_{i}$ o ponto base de $\xi_{i}$ e $d_{i}$ a distância de $N$ a $\gamma\left(t_{i}\right)$. Note que $d\left(p_{i}, p\right) \leq$ $d\left(p_{i}, \gamma\left(t_{i}\right)\right)+d\left(\gamma\left(t_{i}\right), p\right)<d_{i}+t_{i} \leq 2 t_{1}$. Portanto os $\xi_{i}$ então contidos no conjunto compacto $\bigcup_{q \in N, d(q, p) \leq 2 t_{1}} \nu_{q}^{1} N$. Passado para uma subsequencia, podemos assumir que $\left\{\xi_{i}\right\}_{i \in \mathbb{N}}$ converge para algum $\xi_{0} \in \nu N$.

Assuma que $\xi=\xi_{0}$. Seja $d_{i}$ a distância de $N$ a $\gamma\left(t_{i}\right)$. Como $t_{i} \xi$ e $d_{i} \xi_{i}$ tendem a $s_{0} \xi$ e $\exp ^{\perp}\left(t_{i} \xi\right)=$ $\exp ^{\perp}\left(d_{i} \xi_{i}\right)$ para todo $i \in \mathbb{N}$, temos que $s_{0} \xi$ é um ponto crítico de $\exp ^{\perp}$. Então $\gamma\left(s_{0}\right)$ é um ponto focal de $N$ ao longo de $\gamma$. 
Agora assuma que $\xi \neq \xi_{0}$. Seja $\gamma_{0}$ a $N$-geodésica com velocidade inicial $\xi_{0}$. Como $\gamma_{i}$ realiza a distância de $N$ a $\gamma\left(t_{i}\right)$, segue que $\gamma_{0}$ realiza a distância de $N$ a $\gamma\left(s_{0}\right)$. Então há duas $N$-geodésicas distintas realizando a distância de $N$ a $\gamma\left(s_{0}\right)$, concluindo um sentido da demonstração.

Assuma que $\left.\gamma\right|_{\left[0, s_{0}\right]}$ é minimizante. Assuma primeiramente que $\gamma\left(s_{0}\right)$ é ponto focal de $N$ ao longo de $\gamma$. Segue da Proposição 1.2.1 que $\rho_{N}(\xi)=s_{0}$.

Agora assuma que existe outra $N$-geodésica, $\eta:\left[0, s_{0}\right] \rightarrow M$ realizando a distância de $N$ a $\gamma\left(s_{0}\right)$. Assuma por contradição que $\rho_{N}(\xi)=s_{1}>s_{0}$. Seja $c:\left[0, s_{1}\right] \rightarrow M$ a curva suave por partes definida por $c(s)=\eta(s)$ se $0 \leq s \leq s_{0}$ e $c(s)=\gamma(s)$ se $s_{0} \leq s \leq s_{1}$. Claramente $c$ não é diferencial em $s_{0}$, o que contradiz o fato de $c$ realizar a distância de $N$ a $\gamma\left(s_{1}\right)$.

\subsubsection{Distância focal de órbitas de representações}

Nessa subsecção vamos aplicar a Proposição 1.2.2 ao caso particular onde $M$ é uma esfera de raio um e $N$ é uma órbita de uma ação isométrica por um grupo compacto para chagar ao Corolário 1.2.5. Mas antes vamos apresentar algumas definições.

A norma do supremo de uma aplicação linear $T$ entre dois espaços espaços vetoriais normados $\left(U_{1},\|\cdot\|_{1}\right)$ e $\left(U_{2},\|\cdot\|_{2}\right)$ é definida como

$$
\|T\|_{\infty}=\sup \left\{\|T(u)\|_{2}: u \in U_{1},\|u\|_{1}=1\right\} .
$$

Similarmente, a norma do supremo de um aplicação bilinear $F: U_{1} \times U_{1} \rightarrow U_{2}$ é

$$
\|F\|_{\infty}=\sup \left\{\|F(u, v)\|_{2}: u, v \in U_{1},\|u\|_{1}=\|v\|_{1}=1\right\} .
$$

Agora mostramos que a norma do supremo da segunda forma fundamental de uma subvariedade em um ponto é igual à maior curvatura principal nesse mesmo ponto.

Proposição 1.2.3. Seja $N$ uma subvariedade de $(M, g)$. Então existem $u \in T_{p}^{1} N$ e $\xi \in \nu_{p}^{1} N$ tais que

$$
\left\|\mathrm{II}_{p}\right\|_{\infty}=\left\|\mathrm{II}_{p}(u, u)\right\|=\left\|A_{\xi}\right\|_{\infty}=\sup _{\zeta \in \nu_{p}^{1} N}\left\|A_{\zeta}\right\|_{\infty} .
$$

Demonstração. Começamos mostrando que $\sup _{\zeta \in \nu_{p}^{1} N}\left\|A_{\zeta}\right\|_{\infty} \leq\left\|\mathrm{II}_{p}\right\|_{\infty}$. Seja $\zeta \in \nu_{p}^{1} N$ qualquer e seja $v \in T_{p}^{1} N$ tal que $\left\|A_{\zeta}(v)\right\|=\left\|A_{\zeta}\right\|_{\infty}$. Como $A_{\zeta}$ é simétrico, temos que $\left\|A_{\zeta}\right\|_{\infty}=|\mathcal{K}|$ onde $\mathcal{K}$ é o autovalor de $A_{\zeta}$ com maior valor absoluto e $v$ é um autovetor de $A_{\zeta}$ com autovalor associado $\mathcal{K}$. Então temos que

$$
\begin{aligned}
\left\|A_{\zeta}\right\|_{\infty} & =\left\|A_{\zeta}(v)\right\| \\
& =\left|g\left(A_{\zeta}(v), v\right)\right| \\
& =\left|g\left(\mathrm{II}_{p}(v, v), \zeta\right)\right| \\
& \leq\left\|\mathrm{II}_{p}(v, v)\right\| \\
& \leq\left\|\mathrm{II}_{p}\right\|_{\infty} .
\end{aligned}
$$

Agora construímos $u$ e $\xi$. Tome $u, u^{\prime} \in T_{p}^{1} N$ tais que $\left\|\mathrm{II}_{p}\left(u, u^{\prime}\right)\right\|=\left\|\mathrm{II}_{p}\right\|_{\infty}$ e seja $\xi=\frac{\operatorname{II}_{p}\left(u, u^{\prime}\right)}{\left\|\mathrm{II}_{p}\left(u, u^{\prime}\right)\right\|}$. Vamos mostrar que $\left\|A_{\xi}\right\|_{\infty}=\sup _{\zeta \in \nu_{p}^{1} N}\left\|A_{\zeta}\right\|_{\infty}=\left\|\mathrm{II}_{p}\right\|_{\infty}$ e que $u^{\prime}= \pm u$. Primeiramente note que

$$
\begin{aligned}
\left\|\mathrm{II}_{p}\right\|_{\infty} & =\left\|\mathrm{II}_{p}\left(u, u^{\prime}\right)\right\| \\
& =\left|g\left(\operatorname{II}_{p}\left(u, u^{\prime}\right), \xi\right)\right| \\
& =\left|g\left(A_{\xi}(u), u^{\prime}\right)\right| \\
& \leq\left\|A_{\xi}(u)\right\| \\
& \leq\left\|A_{\xi}\right\|_{\infty} .
\end{aligned}
$$


Como $\sup _{\zeta \in \nu_{p}^{1} N}\left\|A_{\zeta}\right\|_{\infty} \leq\left\|\mathrm{II}_{p}\right\|_{\infty}$, devemos ter que $\left\|A_{\xi}\right\|_{\infty}=\sup _{\zeta \in \nu_{p}^{1} N}\left\|A_{\zeta}\right\|_{\infty}=\left\|\mathrm{II}_{p}\right\|_{\infty}$. Então as desigualdades (1.1) e (1.2) são igualdades. Por Cauchy-Schwartz temos que o fato dessa primeira desigualdade ser uma igualdade implica que $A_{\xi}(u)$ e paralelo a $u^{\prime}$. Como $A_{\xi}$ é simétrico, o fato dessa segunda desigualdade ser uma igualdade implica que $u$ é um autovetor de $A_{\xi}$. Segue desses dois fatos que $u$ é paralelo a $u^{\prime}, \operatorname{logo} u= \pm u^{\prime}$.

Definimos o raio focal da subvariedade mergulhada $N \subset M$ como

$$
R_{f}(N)=\inf \left\{\|\xi\|: \xi \in \nu N \text { é ponto crítico de } \exp ^{\perp}\right\} .
$$

Proposição 1.2.4. Seja $K$ um grupo compacto agindo na esfera $S^{n}$ de raio um por isometrias. Seja $p \in S^{n}$ qualquer. Então

$$
R_{f}(K p)=\operatorname{arccot}\left(\left\|\mathrm{II}_{p}\right\|_{\infty}\right),
$$

onde $\mathrm{II}_{p}$ é a segunda forma fundamental fundamental de Kp em $p$.

Demonstração. Como $K$ age transitivamente em $K p$ por isometrias de $S^{n}$ temos que

$$
R_{f}(K p)=\inf \left\{\|\xi\|: \xi \in \nu_{p} N \text { é ponto crítico de } \exp ^{\perp}\right\} .
$$

Seja $\xi \in \nu_{p}^{1} K p$, então $s_{0} \xi$ é ponto crítico de $\exp ^{\perp}$ se, e somente se, $\exp ^{\perp}\left(s_{0} \xi\right)$ for ponto focal ao longo da $K p$-geodésica $\gamma(s)=\exp ^{\perp}(s \xi)$. Seja $J$ um $K p$-campo de Jacobi ao longo de $\gamma$. Como estamos interessados nos $K p$-campos de Jacobi que se anulam em algum ponto, podemos assumir sem perda de generalidade que $J(s) \perp \gamma^{\prime}(s)$ para todo o $s \in \mathbb{R}$. Seja $u=J(0)$. Pelas condições iniciais

$$
u \in T K p \quad \text { e } \quad J^{\prime}(0)+A_{\xi}(u) \in \nu K p
$$

temos que $J^{\prime}(0)=v+\zeta$, onde $v=-A_{\xi}(u)$ e $\zeta \in \nu_{p} K p$. Como $J(s) \perp \gamma^{\prime}(s)$ para todo o $s \in \mathbb{R}$, a equação de Jacobi fica:

$$
-J^{\prime \prime}(s)+\mathcal{R}\left(\gamma^{\prime}(s), J(s)\right) \gamma^{\prime}(s)=-J^{\prime \prime}(s)-J(s)=0 .
$$

Então devemos ter:

$$
J(s)=\cos (s) u(s)+\sin (s)(v(s)+\zeta(s)),
$$

onde $u(s), v(s)$ e $\zeta(s)$ os campos ao longo de $\gamma$ obtidos por transporte paralelo de $u, v$ e $\zeta$ respectivamente.

Como $u(s) \perp \zeta(s)$ para todo o $s \in \mathbb{R}$, temos que $J$ só se anula se $u=0$ ou se $\zeta=0$. Primeiramente assuma que $u=0$. Segue que o menor $s_{0}>0$ tal que $J\left(s_{0}\right)=0$ é $s_{0}=\pi$. Agora assuma que $\zeta=0$. Então $J$ só se anula se $v$ for paralelo a $u$, isso é, $u$ deve ser autovetor de $A_{\xi}$. Assuma que $u$ é autovetor de $A_{\xi}$, seja $\kappa$ o autovalor associado a $u$ e seja $s_{0}$ o menor número positivo tal que $J\left(s_{0}\right)=0$. Então temos que

$$
J\left(s_{0}\right)=\left(\cos \left(s_{0}\right)-\kappa \sin \left(s_{0}\right)\right) u\left(s_{0}\right)=0 .
$$

Segue que

$$
\kappa=\frac{\cos \left(s_{0}\right)}{\sin \left(s_{0}\right)}=\cot \left(s_{0}\right) .
$$

Como arccot é decrescente, temos que

$$
s_{0}=\operatorname{arccot}(\kappa) \geq \operatorname{arccot}\left(\left\|A_{\xi}\right\|_{\infty}\right) \geq \operatorname{arccot}\left(\left\|\mathrm{II}_{p}\right\|_{\infty}\right) .
$$

Note que a igualdade nas inequações acima é atingida, bastando tomar $\xi$ e $u$ como na Proposição 1.2.3. Segue que $R_{f}(K p)=\operatorname{arccot}\left(\left\|\mathrm{II}_{p}\right\|_{\infty}\right)$.

A Proposição 1.2.4 torna fácil encontrar geodésicas que atendem ao primeiro critério listado na 
Proposição 1.2.2. Portanto, para encontrar uma cota inferior para o diâmetro do quociente de uma esfera através da Proposição 1.2.2 falta apenas considerar o segundo critério, como formalizado no Corolário abaixo.

Corolário 1.2.5. Seja K um grupo de Lie compacto agindo em $S^{n}$ por isometrias. Seja $p \in S^{n}$ e seja $\gamma:[0, \infty) \rightarrow S^{n}$ uma Kp-geodésica com velocidade de norma um. Tome $\epsilon>0$ tal que

(i) $\epsilon \leq \operatorname{arccot}\left(\left\|\mathrm{II}_{p}\right\|_{\infty}\right) e$

(ii) $\left.\gamma\right|_{[0, \epsilon)}$ não intersecta nenhuma Kp-geodésica minimizante.

Então o diâmetro do espaço de órbitas $S^{n} / K$ é maior ou igual a $\epsilon$.

Demonstração. Da Proposição 1.2.4 temos que não existe ponto focal de $K p$ ao longo de $\left.\gamma\right|_{[0, \epsilon)}$. Usando esse fato e a segunda hipótese, temos que segue da Proposição 1.2 .2 que $\left.\gamma\right|_{[0, \epsilon]}$ realiza a distância de $K p$ a $\gamma(\epsilon)$. Como essa distância é igual à distância de $K p$ a $K \gamma(\epsilon)$, temos que

$$
\operatorname{diam}\left(S^{n} / K\right) \geq d(K p, K \gamma(\epsilon))=\epsilon .
$$

Acreditamos que o lema de Kleiner possa ser usado para estudar a segunda condição listada no corolário acima. Antes de desenvolver melhor essa especulação, apresentamos o Lema de Kleiner (para uma prova, ver $[\mathrm{AB} 15]$ ).

Lema 1.2.6. Seja $K$ um grupo de Lie agindo na variedade riemanniana $M$ por isometrias, seja $\gamma:[0, l] \rightarrow M$ uma geodésica realizando a distância de $K \gamma(0)$ a $K \gamma(l)$. Então

$$
K_{\gamma\left(s_{1}\right)}=\bigcap_{s \in[0, l]} K_{\gamma(s)} \quad \forall s_{1} \in(0, l),
$$

onde $K_{\gamma(s)}$ é o grupo de isotropia em $\gamma(s)$.

Para ver como esse lema se relaciona ao Corolário 1.2.5, tomamos $p \in S^{n}$ e $\gamma:[0, \infty) \rightarrow S^{n}$ uma $K p$-geodésica com velocidade inicial $\xi \in \nu_{p}^{1} K p$. Seja $\epsilon>0$ o maior número que atende as duas condições do Corolário 1.2.5. Segue que $\epsilon=\operatorname{arccot}\left(\left\|\mathrm{II}_{p}\right\|_{\infty}\right)$ ou que existe uma $K p$-geodésica diferente de $\gamma$ que realiza a distância de $K p$ a $q=\gamma(\epsilon)$. Assuma que a segunda situação ocorre. Seja $\eta:[0, \epsilon] \rightarrow S^{n}$ uma $K p$-geodésica diferente de $\gamma$ realizando a distância de $K p$ a $q$. Queremos encontrar uma cota inferior para $\epsilon$. Tomando $k \in K$ tal que $k . p=\eta(0)$, segue do Lema de Kleiner que

$$
K_{\gamma(s)} \subset K_{p} \cap K_{q} \quad \text { e } \quad K_{\eta(s)} \subset k K_{p} k^{-1} \cap K q \quad \forall s \in(0, \epsilon) .
$$

Para $s>0$ suficientemente pequeno, o grupo de isotropia em $\gamma(s)$ é igual ao grupo de isotropia em $\xi$ da representação de isotropia em $p$. Especulamos que, conhecendo os grupos de isotropia da ação, seja possível fazer uma escolha conveniente de $\xi$ que restrinja os possíveis $K_{q}$, e consequentemente os possíveis $k$. Então, usando que $d(p, k . p) \leq 2 \epsilon$, esperamos que seja possível encontrar uma cota inferior para $\epsilon$.

\subsection{Representações de grupos de Lie compactos}

Ações isométricas de um grupo compacto $K$ na esfera $S^{n}$ podem ser estendidas para representação ortogonais de $K \mathrm{em} \mathbb{R}^{n+1}$. Aqui apresentamos propriedades das representações ortogonais de grupos compactos que serão usadas nas provas dos principais teoremas desse texto. Começamos apresentando como representações ortogonais induzem representações unitárias e vice versa, para então classificarmos representações irredutíveis ortogonais e unitárias nos tipos real, complexo e quaterniônico. Em seguida apresentamos algumas fórmulas relativas à segunda forma fundamental de órbitas de representações que serão usadas no Capítulo 2. 


\subsubsection{Classificação nos tipos real, complexo e quaterniônico}

Seguindo o capítulo II.6 de [BtD95], apresentamos os conceito de realificação e complexificação de representações e a classificação de representações ortogonais e unitárias nos tipos real, complexo e quaterniônico.

A realificação do espaço vetorial complexo $W$ é o espaço vetorial real $W_{\mathbb{R}}$ que coincide com $W$ como grupo aditivo e tem multiplicação por escalares dada pela restrição da multiplicação por escalares de $W$ a $\mathbb{R}$. Se $W$ for uma representação unitária de $K$, então $W_{\mathbb{R}}$ é uma representação ortogonal de $K$ se tomarmos o produto simétrico natural dado por $\left\langle w_{1}, w_{2}\right\rangle_{\mathbb{R}}=R e\left\langle w_{1}, w_{2}\right\rangle$ para todo $w_{1}, w_{2} \in W$, onde $\operatorname{Re}\left\langle w_{1}, w_{2}\right\rangle$ é a parte real de $\left\langle w_{1}, w_{2}\right\rangle$.

A complexificação do espaço vetorial real $V$ é o grupo aditivo $\mathbb{C} \otimes_{\mathbb{R}} V$ com multiplicação por complexos dada por $z_{1}\left(z_{2} \otimes v\right)=z_{1} z_{2} \otimes v$ para todos $z_{1}, z_{2} \in \mathbb{C}, v \in V$. Se $V$ for uma representação ortogonal de $K$, então $\mathbb{C} \otimes V$ também é representação de $K$ se tomarmos $k$. $(z \otimes v)=z \otimes k . v$ para todo $k \in K, z \in \mathbb{C}, v \in V$. Essa representação é ortogonal pelo produto hermiano natural dado por

$$
\left(1 \otimes v_{1}+i \otimes v_{2}, 1 \otimes v_{1}^{\prime}+i \otimes v_{2}^{\prime}\right)_{\mathbb{C}}=\left(v_{1}, v_{1}^{\prime}\right)+\left(v_{2}, v_{2}^{\prime}\right)+i\left(v_{2}, v_{1}^{\prime}\right)-i\left(v_{1}, v_{2}^{\prime}\right)
$$

para todo $v_{1}, v_{2}, v_{1}^{\prime}, v_{2}^{\prime} \in V$.

Proposição 1.3.1. Seja $V$ uma representação ortogonal de $K$ e $W$ uma representação unitária de K. Então

$$
(\mathbb{C} \otimes V)_{\mathbb{R}} \cong V \oplus V \quad \text { e } \quad \mathbb{C} \otimes W_{\mathbb{R}} \cong W \oplus W^{*} .
$$

Demonstração. Basta verificar que as aplicações

$$
\begin{aligned}
\alpha:(\mathbb{C} \otimes V)_{\mathbb{R}} & \rightarrow V \oplus V & \beta: \mathbb{C} \otimes W_{\mathbb{R}} & \rightarrow W \oplus W^{*} \\
\left(1 \otimes v_{1}+i \otimes v_{2}\right) & \mapsto\left(v_{1}, v_{2}\right) & (z \otimes w) & \mapsto(z w, z\langle\cdot, w\rangle)
\end{aligned}
$$

são isomorfismos de representações.

Uma estrutura real em uma representação unitária $W$ de $K$ é uma aplicação $\sigma: W \rightarrow W$ tal que:

(i) $\sigma$ é antilinear, isso é, $\sigma$ é $\mathbb{R}$-linear e $\sigma(z w)=\bar{z} \sigma(w)$ para todo $z \in \mathbb{C}, w \in W$;

(ii) $\sigma$ comuta com a ação de $K$, ou seja, $\sigma(k . w)=k . \sigma(w)$ para todo $k \in K, w \in W$;

(iii) $\sigma$ é involutiva, isso é, $\sigma^{2}=I d$, a identidade em $W$.

A complexificação $\mathbb{C} \otimes V$ da representação ortogonal $V$ tem estrutura real canônica $\sigma$ dada por $\sigma(z \otimes v)=\bar{z} \otimes v$. Note que o espaço vetorial real dos vetores fixados por $\sigma$ é uma subrepresentação real de $\mathbb{C} \otimes V$ que pode ser identificada com $V$ pela aplicação $1 \otimes v \mapsto v$.

De maneira geral, uma estrutura real $\sigma$ em $W$ decompõe $W_{\mathbb{R}}$ em duas subrepresentações reais $W_{+}$e $W_{-}$, onde $W_{+}$é formada pelos autovetores de $\sigma$ com autovalor 1 e é chamada de forma real, e $W_{-}$é formada pelos autovetores de $\sigma$ com autovalor -1 . Da antilinearidade de $\sigma$ segue que a multiplicação pelo número complexo unitário puro $i$ é um isomorfismo $\mathbb{R}$-linear entre essas duas subrepresentações.

Proposição 1.3.2. Seja $W$ uma representação unitária irredutível de $K$ com produto hermitiano $\langle$,$\rangle e estrutura real \sigma$. Então $\sigma$ é uma isometria de $W_{\mathbb{R}}$ e é simétrica (em relação ao produto simétrico $\left.\langle,\rangle_{\mathbb{R}}\right)$.

Demonstração. Note que basta mostrar que $W_{+}$e $W_{-}$são ortogonais pelo produto $\langle,\rangle_{\mathbb{R}}$. Seja $H$ o produto hermitiano em $W$ definido por $H\left(w_{1}, w_{2}\right)=\overline{\left\langle\sigma\left(w_{1}\right), \sigma\left(w_{2}\right)\right\rangle}$ para todo $w_{1}, w_{2} \in W$. Como $H$ é $K$-invariante e $W$ é irredutível, segue do Lema de Schur que $H$ é múltiplo positivo de $\langle$,$\rangle . Para$ qualquer $w \in W_{+}$temos que $H(w, w)=\langle w, w\rangle$, então $H=\langle$,$\rangle . Logo para quaisquer w_{1}, w_{2} \in W_{+}$ temos que

$$
\left\langle w_{1}, w_{2}\right\rangle=H\left(w_{1}, w_{2}\right)=\overline{\left\langle w_{1}, w_{2}\right\rangle},
$$


segue que $\left\langle w_{1}, w_{2}\right\rangle$ é real. Usando esse fato temos que

$$
\left\langle w_{1}, i w_{2}\right\rangle_{\mathbb{R}}=\operatorname{Re}\left(-i\left\langle w_{1}, w_{2}\right\rangle\right)=0,
$$

e portanto $W_{+} \perp i W_{+}$. Como $W_{-}=i W_{+}$, segue que $W_{+} \perp W_{-}$.

Uma estrutura quaterniônica $\mathcal{J}$ em uma representação unitária $W$ de $K$ é um endomorfismo antilinear, que comuta com a ação de $K$ em $W$, tal que $\mathcal{J}^{2}=-I d$. Usando a estrutura quaterniônica podemos definir uma multiplicação por quatérnions em $W$ por $\left(z_{1}+z_{2} j\right) w=z_{1} w+z_{2} \mathcal{J}(w)$ para $z_{1}, z_{2} \in \mathbb{C}, w \in W$.

Definição 1.3.3. Uma representação unitária irredutivel $W$ é dita de tipo

(i) real se $W$ tem estrutura real,

(ii) complexa se a representação dual $W^{*}$ não for isomorfa a $W$,

(iii) quaterniônica se $W$ tem estrutura quaterniônica.

Mostraremos que toda representação unitária irredutível de um grupo compacto é de um, e apenas um, desses tipos. Para mostrar esse fato precisamos da caracterização dada pela proposição abaixo.

Proposição 1.3.4. Uma representação unitária irredutivel $W$ de um grupo compacto $K$ é de tipo real (resp. quaterniônico) se, e somente se, existir uma forma bilinear não singular simétrica (resp. antissimétrica) $K$-invariante $F: W \times W \rightarrow \mathbb{C}$.

Demonstração. Primeiramente suponha que existe uma forma bilinear $K$-invariante não singular $F$ com $F\left(w_{1}, w_{2}\right)=\varepsilon F\left(w_{2}, w_{1}\right)$ para todo $w_{1}, w_{2} \in W$, onde $\varepsilon= \pm 1$. Seja $f: W \rightarrow W$ a aplicação definida por $F\left(w_{1}, w_{2}\right)=\left\langle w_{1}, f\left(w_{2}\right)\right\rangle$. Segue que $f$ é antilinear, comuta com a ação de $K$ e é injetora. Agora note que

$$
\left\langle w_{1}, f\left(w_{2}\right)\right\rangle=F\left(w_{1}, w_{2}\right)=\varepsilon F\left(w_{2}, w_{1}\right)=\varepsilon\left\langle w_{2}, f\left(w_{1}\right)\right\rangle=\varepsilon \overline{\left\langle f\left(w_{1}\right), w_{2}\right\rangle} .
$$

Temos então que

$$
\left\langle w_{1}, f^{2}\left(w_{2}\right)\right\rangle=\varepsilon \overline{\left\langle f\left(w_{1}\right), f\left(w_{2}\right)\right\rangle}=\left\langle f^{2}\left(w_{1}\right), w_{2}\right\rangle,
$$

$\operatorname{logo} f^{2}$ é simétrico. Como $\left\langle w, \varepsilon f^{2}(w)\right\rangle=\overline{\langle f(w), f(w)\rangle}$ segue que $\varepsilon f^{2}$ é definido positivo. Como $W$ é irredutível e $\varepsilon f^{2}$ é $\mathbb{C}$-linear e comuta com a ação de $K$, segue do Lema de Schur que $\varepsilon f^{2}=\lambda I d$ para algum $\lambda>0$. Temos então que $\frac{1}{\sqrt{\lambda}} f$ é estrutura real (resp. quaterniônica) em $W$ se $\varepsilon=1$ (resp. $\varepsilon=-1$ ).

Agora suponha que $W$ é de tipo real (resp. quaterniônico) e seja $\mathcal{E}$ a estrutura real (resp. quaterniônica) em $W$. Como $\mathcal{E}$ é antilinear e bijetiva, a aplicação $F: W \times W \rightarrow \mathbb{C}$ definida por $F\left(w_{1}, w_{2}\right)=\left\langle w_{1}, \mathcal{E}\left(w_{2}\right)\right\rangle$ é $\mathbb{C}$-bilinear, não singular e $K$-invariante. Como $W$ é irredutível, temos que $F$ é simétrico ou antissimétrico, ou seja, $F\left(w_{1}, w_{2}\right)=\varepsilon F\left(w_{2}, w_{1}\right)$ para todo o $w_{1}, w_{2} \in W$, onde $\varepsilon= \pm 1$. De maneira similar a como foi feito na primeira parte dessa prova, mostra-se que $\left\langle w_{1}, \mathcal{E}\left(w_{2}\right)\right\rangle=\varepsilon \overline{\left\langle\mathcal{E}\left(w_{1}\right), w_{2}\right\rangle}$. Então temos que

$$
\left\langle w, \mathcal{E}^{2}(w)\right\rangle=\varepsilon \overline{\langle\mathcal{E}(w), \mathcal{E}(w)\rangle} .
$$

Como $\langle\mathcal{E}(w), \mathcal{E}(w)\rangle>0$ temos que $\varepsilon=1$ (resp. $\varepsilon=-1$ ) se $\mathcal{E}$ for uma estrutura real (resp. quaterniônica) e portanto $F$ é simétrico (resp. antissimétrico).

Proposição 1.3.5. Toda representação unitária irredutível de um grupo compacto é de tipo real, complexo ou quaterniônico, e não pode ser de mais de um desses tipos.

Demonstração. Seja $W$ uma representação unitária irredutível de um grupo compacto $K$. Se $W$ não for isomorfo a $W^{*}$, segue que $W$ é de tipo complexo. Agora assuma que $W$ é isomorfo a $W^{*}$. Seja $\phi$ um isomorfismo entre $W$ e $W^{*}$. Tomando

$$
\phi_{S}=\frac{\phi+\phi^{*}}{2} \quad \text { e } \quad \phi_{A}=\frac{\phi-\phi^{*}}{2},
$$


temos que $\phi=\phi_{S}+\phi_{A}$, que $\phi_{S}$ e $\phi_{A}$ são $K$-invariantes, e que $\phi_{S}$ (resp. $\phi_{A}$ ) é simétrico (resp. antissimétrico).

Como $W$ é irredutível, segue do Lema de Schur que $\phi_{A}=0$ ou $\phi_{S}=0$. Se $\phi_{A}=0\left(\right.$ resp. $\left.\phi_{S}=0\right)$ a forma bilinear em $W$ definida por $\left(w_{1}, w_{2}\right) \mapsto \phi\left(w_{1}\right)\left(w_{2}\right)$ é simétrica (resp. antissimétrica) e portanto, pela Proposição 1.3.4, $W$ é de tipo real (resp. quaterniônico). O fato que $W$ não pode ser de mais de um desses tipos segue de que formas bilineares $K$-invariantes não singulares definem isomorfismo de $W$ a $W^{*}$ e, pelo Lema de Schur, todo isomorfismo de $W$ a $W^{*}$ é múltiplo de $\phi$.

Existe um análogo dessa classificação para representações ortogonais irredutíveis.

Definição 1.3.6. Uma representação ortogonal irredutivel $V$ é dita de tipo

(i) real se a complexificação de $V$ for irredutivel,

(ii) complexo se $V$ for a realificaçâo de uma representação unitária irredutivel de tipo complexo,

(iii) quaterniônico se $V$ for a realificação de uma representação unitária irredutivel de tipo quaterniônico.

Note que se $V$ for uma representação ortogonal irredutível de tipo real, então $V$ não pode ser a realificação de uma representação unitária, já que se $V=W_{\mathbb{R}}$ para alguma representação unitária $W$ temos pela Proposição 1.3.1 que $\mathbb{C} \otimes V=\mathbb{C} \otimes W_{\mathbb{R}}$ não é irredutível.

Proposição 1.3.7. Toda representação ortogonal irredutivel de um grupo de Lie compacto é de tipo real, complexo ou quaterniônico, e não pode ser de mais de um desses tipos.

Demonstração. Seja $V$ uma representação ortogonal irredutível de um grupo compacto $K$ e $W$ a complexificação de $V$. Se $W$ for irredutível, segue que $V$ é de tipo real. Assuma agora que $W$ não é irredutível, então $W=\bigoplus_{i=1}^{n} W_{i}$ onde todos os $W_{i}$ são representações unitárias irredutíveis. Pela Proposição 1.3.1 temos $V \oplus V \cong W_{\mathbb{R}} \cong \bigoplus_{i=1}^{n}\left(W_{i}\right)_{\mathbb{R}}$. Então como $V$ é irredutível temos que $n=2$ $\mathrm{e}\left(W_{1}\right)_{\mathbb{R}} \cong V \cong\left(W_{2}\right)_{\mathbb{R}}$.

Se $W_{1}$ não for isomorfo a $W_{1}^{*}$, temos que $W_{1}$ é de tipo complexo, e portanto $V$ é de tipo complexo. Agora assuma que $W_{1} \cong W_{1}^{*}$. Como $\left(W_{1}\right)_{\mathbb{R}}$ é irredutível temos que $W_{1}$ não pode ser de tipo real, então segue que $W_{1}$ é de tipo quaterniônico e portanto $V$ é de tipo quaterniônico.

\subsubsection{Segunda forma fundamental das órbitas de uma representação}

Aqui vamos mostrar algumas identidades que usaremos para estudar a segunda forma fundamental de órbitas de representações no Capítulo 2. Seja $V$ uma representação ortogonal do grupo de Lie compacto $K$. Como as órbitas da representação de $K$ e da representação da imagem de $K$ no grupo ortogonal $\mathrm{O}(V)$ de $V$ são as mesmas, vamos assumir que $K \subset \mathrm{O}(V)$. A álgebra de Lie $\mathfrak{k}$ de $K$ é uma subálgebra da álgebra $\mathfrak{o}(V)$ de endomorfismos lineares antissimétricos de $V$. Como estamos interessados nas restrições de representações à esferas, consideraremos a órbita $K p$ como subvariedade da esfera $S_{p}(V)$, onde $S_{p}(V) \subset V$ é a esfera com centro na origem contendo $p \in V$. Para simplificar a notação, identificamos o espaço tangente a $p \in V \operatorname{com} V$ pela isometria

$$
\begin{aligned}
\tau_{p}: V & \rightarrow T_{p} V \\
v & \left.\mapsto \frac{d}{d s}(p+s v)\right|_{s=0} .
\end{aligned}
$$

Como estamos identificando $K$ com sua imagem em $\mathrm{O}(V)$, a exponencial de $K$ é a restrição da exponencial de $\mathrm{O}(V)$ e portanto

$$
\left.\frac{d}{d s} \operatorname{Exp}(s x) \cdot p\right|_{s=0}=\left.\frac{d}{d s}\left(p+\sum_{n=1}^{\infty} \frac{(s x)^{n} \cdot p}{n !}\right)\right|_{s=0}=x \cdot p \forall x \in \mathfrak{k}, p \in V .
$$

Então temos que

$$
T_{p} K p=\{x \cdot p: x \in \mathfrak{k}\}=\mathfrak{k} \cdot p
$$


e

$$
\nu_{p} K p=(\mathfrak{k} . p \oplus \mathbb{R} p)^{\perp},
$$

onde $(\mathfrak{k} . p \oplus \mathbb{R} p)^{\perp}$ complemento ortogonal a $\mathfrak{k} . p \oplus \mathbb{R} p$ em $V$.

Seja $(\cdot)^{\perp}$ a projeção ortogonal em $(\mathfrak{k} p \oplus \mathbb{R} p)^{\perp}$ e sejam $x, y \in \mathfrak{k}$. Tomando $\tilde{x}$ e $\tilde{y}$ os campos suaves em $V$ definidos por $p \mapsto x$. $p$ e $p \mapsto y . p$ respectivamente, temos que

$$
\mathrm{II}_{p}(x \cdot p, y \cdot p)=\operatorname{II}_{p}\left(\tilde{x}_{p}, \tilde{y}_{p}\right)=\left(\nabla_{\tilde{x}} \tilde{y}\right)_{p}^{\perp} .
$$

Note que $\left(\nabla_{\tilde{x}} \tilde{y}\right)_{p}=D_{p} y(x . p)$ onde $D_{p} y$ é a derivada usual de $y$. Como $y$ é linear temos que $D_{p} y(x . p)=y . x . p$. Usando o fato da segunda forma fundamental ser simétrica temos que

$$
\mathrm{II}_{p}(x \cdot p, y \cdot p)=(x \cdot y \cdot p)^{\perp} .
$$

A segunda forma fundamental das órbitas passando por $r p$ para $r>0$ e $p \in S^{n}$ fixo é inversamente proporcional a $r$, isso é:

Proposição 1.3.8. Sejam $p \in V$ e $r>0$. Então temos que

$$
\mathrm{II}_{r p}=\frac{1}{r} \mathrm{II}_{p}
$$

Demonstração. Seja $x, y \in \mathfrak{k}$. Por (1.4) temos:

$$
\begin{aligned}
\mathrm{II}_{r p}(x . r p, y \cdot r p) & =(x \cdot y \cdot r p)^{\perp} \\
& =r(x \cdot y \cdot p)^{\perp} \\
& =r \mathrm{II}_{p}(x \cdot p, y \cdot p) \\
& =\frac{1}{r} \mathrm{II}_{p}(r x \cdot p, r y \cdot p) .
\end{aligned}
$$

\section{4 Álgebras de Lie compactas e suas representações}

A identidade (1.4) relaciona a segunda forma fundamental de $K p$ com a representação de $\mathfrak{k}$ em $V$. Aqui trataremos de propriedades das álgebras de Lie compactas e das suas representações que serão utilizadas no Capítulo 2. Começamos apresentado propriedades das álgebras de Lie compactas e de suas complexificações. Na Subseç̧ão 1.4.2 apresentamos propriedades algébricas e geométricas de representações de álgebras de Lie compactas. O principal objetivo dessa secção é mostrar a Proposição 1.4.2 e as identidades (1.7) e (1.8).

\subsection{1 Álgebras de Lie compactas e suas complexificações}

Uma álgebra de Lie real compacta é uma álgebra de Lie isomorfa a álgebra de Lie de um grupo compacto. Todo grupo compacto tem uma métrica bi-invariante (invariante pelo produto por elementos do grupo à esquerda e à direita). Essa métrica induz um produto simétrico invariante na álgebra de Lie do grupo. Como o complemento ortogonal de um ideal por um produto simétrico invariante também é um ideal, temos que a representação adjunta de álgebras de Lie compactas são completamente redutíveis. Isso é, se k é a álgebra de Lie do grupo compacto $K$, então podemos escrever

$$
\mathfrak{k}=\bigoplus_{i=1}^{m} \mathfrak{k}_{i} \oplus \mathfrak{a}
$$

onde os $\mathfrak{k}_{i}$ são ideais simples de $\mathfrak{k}$ e a é uma álgebra de Lie abeliana. A soma dos $\mathfrak{k}_{i}$ 's é chamada parte semisimples de $\mathfrak{k}$ e será denotada por $\mathfrak{k}_{s}$, e a é a parte abeliana de $\mathfrak{k}$. 
Proposição 1.4.1. A forma de Cartan-Killing $B$ de uma álgebra de Lie compacta $\mathfrak{k}$ é semidefinida negativa, e $B(x, x)=0$ se, e somente se, $x \in \mathfrak{a}$.

Demonstração. Seja $Q$ um produto simétrico invariante em $\mathfrak{k}$ e seja $\left\{y_{1}, \cdots, y_{n}\right\}$ uma base ortonormal de $\mathfrak{k}$ com respeito a $Q$. Tomando $x \in \mathfrak{k}$ qualquer temos que

$$
\begin{aligned}
B(x, x) & =\operatorname{tr}(\operatorname{ad}(x) \circ \operatorname{ad}(x)) \\
& =\sum_{i=1}^{n} Q\left(\operatorname{ad}(x) a d(x) y_{i}, y_{i}\right) \\
& =-\sum_{i=1}^{n} Q\left(\operatorname{ad}(x) y_{i}, a d(x) y_{i}\right) \\
& \leq 0 .
\end{aligned}
$$

Note que $B(x, x)=0$ se, e somente se, $\operatorname{ad}(x)=0$.

A complexificação da álgebra de Lie $\mathfrak{k}$ é o espaço vetorial complexo $\mathbb{C} \otimes \mathfrak{k}$ com o colchete definido por

$$
\left[z_{1} \otimes x_{1}, z_{2} \otimes x_{2}\right]=z_{1} z_{2} \otimes\left[x_{1}, x_{2}\right] \forall z_{1}, z_{2} \in \mathbb{C}, x_{1}, x_{2} \in \mathfrak{k} .
$$

Uma estrutura real em uma álgebra de Lie complexa g é uma aplicação antilinear e involutiva $\varepsilon: \mathfrak{g} \rightarrow \mathfrak{g}$ tal que $[\varepsilon(e), \varepsilon(f)]=\varepsilon([e, f])$ para todo $e, f \in \mathfrak{g}$. Álgebras de Lie complexas podem ter várias estruturas reais. Como nesses texto sempre tomaremos álgebras de Lie complexas $\mathfrak{g}=\mathbb{C} \otimes \mathfrak{k}$ onde $\mathfrak{k}$ é compacto, tomaremos a estrutura real natural em $\mathfrak{g}$ dada por $z \otimes x \mapsto \bar{z} \otimes x$. O isomorfismo $x \mapsto 1 \otimes x$ identifica $\mathfrak{k}$ com a subálgebra real de $\mathfrak{g}$ formada pelos vetores fixados por essa estrutura real.

Tomando a decomposição de $\mathfrak{k}$ apresentada acima temos que

$$
\mathfrak{g} \cong \bigoplus_{i=1}^{m} \mathfrak{g}_{i} \oplus(\mathbb{C} \otimes \mathfrak{a}),
$$

onde $\mathfrak{g}_{i}=\mathbb{C} \otimes \mathfrak{k}_{i}$. Como $\mathfrak{k}_{i}$ é um ideal simples de $\mathfrak{g}$ temos que $\mathfrak{g}_{i}$ é um ideal simples de $\mathfrak{g}$. A soma dos $\mathfrak{g}_{i}$ 's é a parte semisimples de $\mathfrak{g}$ e será escrita como $\mathfrak{g}_{s}, \mathbb{C} \otimes \mathfrak{a}$ é a parte abeliana de $\mathfrak{g}$.

Seja $\mathfrak{t} \subset \mathfrak{k}$ a álgebra de Lie de um toro maximal de $K$. A complexificação de $\mathfrak{t}$ é uma subálgebra de Cartan $\mathfrak{h} \subset \mathfrak{g}$. Escreveremos $R \subset \mathfrak{h}^{*}$ para as raízes de $\mathfrak{g}$ e $B($,$) para a forma de Cartan-Killing$ em $\mathfrak{g}$. Por abuso de notação também usaremos $B($,$) para o produto simétrico induzido pela forma$ de Cartan-Killing no subespaço real de $\mathfrak{h}^{*}$ gerado por $R$. Para as raízes $\alpha$ de $\mathfrak{g}$ escrevemos

(i) $\mathfrak{g}_{\alpha}$ para o espaço de vetores raízes com raiz associada $\alpha$;

(ii) $\alpha^{\vee}$ para a co-raiz de $\alpha$, isso é $\alpha^{\vee}=\frac{2 \alpha}{B(\alpha, \alpha)}$;

(iii) $h_{\alpha} \in \mathfrak{h}$ para o vetor na parte semisimples de $\mathfrak{g}$ satisfazendo $B\left(h_{\alpha}, h\right)=\alpha^{\vee}(h)$ para todo $h \in \mathfrak{h}$.

Fixe uma polarização de $R$. Escrevemos $R_{+} \subset R$ para o conjunto de raízes positivas de $R$ e $R_{-}$para o conjunto de raízes negativas. Agora vamos provar a existência de uma base para $\mathfrak{k}_{s}$, conhecida como base de Weil, que tem propriedades que serão utilizados no Capítulo 2.

Proposição 1.4.2. Usando a notação acima, temos que para todo $\alpha \in R_{+}$existem vetores $e_{\alpha} \in \mathfrak{g}_{\alpha}$ e $f_{\alpha} \in \mathfrak{g}_{-\alpha}$ tais que

(i) $\left[e_{\alpha}, f_{\alpha}\right]=h_{\alpha}, e$

(ii) $x_{\alpha}=e_{\alpha}-f_{\alpha}, y_{\alpha}=i\left(e_{\alpha}+f_{\alpha}\right) \in \mathfrak{k}$.

Adicionalmente, o conjunto $\left\{x_{\alpha}, y_{\alpha}, i h_{\alpha} \mid \alpha \in R_{+}\right\}$gera a $\mathfrak{k}_{s}$.

Demonstração. A prova será feita por partes. Seja $\varepsilon$ a estrutura real em $\mathfrak{g}$ dada por $z \otimes x \mapsto \bar{z} \otimes x$. Começamos mostrando que $\varepsilon\left(\mathfrak{g}_{\alpha}\right)=\mathfrak{g}_{-\alpha}$ para todo $\alpha \in R$. Seja $H: \mathfrak{g} \times \mathfrak{g} \rightarrow \mathbb{C}$ a forma hermitiana 
dada por $H(x, y)=-B(x, \varepsilon(y))$. Segue da Proposição 1.4.1 que $H$ é definida positiva em $\mathfrak{g}_{s}$. Para todo $t \in \mathfrak{t}$ e $f, e \in \mathfrak{g}$ temos que

$$
\begin{aligned}
H(\operatorname{ad}(t)(e), f) & =-B(\operatorname{ad}(t)(e), \varepsilon(f)) \\
& =B(e, \operatorname{ad}(t)(\varepsilon(f))) \\
& =B(e, \varepsilon([\varepsilon(t), f])) \\
& =B(e, \varepsilon([t, f])) \\
& =-H(e, \operatorname{ad}(t)(f))
\end{aligned}
$$

Portanto $\operatorname{ad}(t)$ é anti-adjunta em relação a $H$ (isso é, a adjunta de $\operatorname{ad}(t)$ é $-\operatorname{ad}(t)$ ). Logo os autovalores de $\operatorname{ad}(t)$ são imaginários puros. Então para todos $\alpha \in R, e \in \mathfrak{g}_{\alpha}$ e $t \in \mathfrak{t}$ temos que

$$
\operatorname{ad}(t)(\varepsilon(e))=\varepsilon([\varepsilon(t), e])=\varepsilon([t, e])=\varepsilon(\alpha(t) e)=-\alpha(t) \varepsilon(e),
$$

$\log 0\left(\mathfrak{g}_{\alpha}\right)=\mathfrak{g}_{-\alpha}$.

Agora vamos mostrar que para todo $\alpha \in R_{+}$e para todo $e \in \mathfrak{g}_{\alpha}$ não nulo existe $r>0$ tal que $[r e,-\varepsilon(r e)]=h_{\alpha}$. Como $\varepsilon(e) \in \mathfrak{g}_{-\alpha}$ temos que $[r e,-\varepsilon(r e)] \in \mathfrak{h} \cap \mathfrak{g}_{s}$. Então basta mostrar que podemos tomar um $r>0$ tal que $B([r e,-\varepsilon(r e)], h)=\alpha^{\vee}(h)$ para todo $h \in \mathfrak{h}$. Pela a invariância da forma de Cartan-Killing temos que

$$
\begin{aligned}
B([r e,-\varepsilon(r e)], h) & =r^{2} B(e,[-\varepsilon(e), h]) \\
& =r^{2} B(e,[h, \varepsilon(e)]) \\
& =r^{2} B(e,-\alpha(h) \varepsilon(e)) \\
& =-\alpha(h) r^{2} B(e, \varepsilon(e)) \\
& =\alpha(h) r^{2} H(e, e) .
\end{aligned}
$$

Como $H$ é positivo definido em $\mathfrak{g}_{s}$, temos que $H(e, e)>0$. Então podemos podemos tomar $r=$ $\sqrt{\frac{2}{B(\alpha, \alpha) H(e, e)}}$.

Agora estamos prontos para definir os $e_{\alpha}$ 's e os $f_{\alpha}$ 's. Para todo $\alpha \in R_{+}$escolha um $e_{\alpha}^{\prime} \in \mathfrak{g}_{\alpha}$ não nulo e tome $e_{\alpha}=\sqrt{\frac{2}{B(\alpha, \alpha) H\left(e_{\alpha}^{\prime}, e_{\alpha}^{\prime}\right)}} e_{\alpha}^{\prime}, f_{\alpha}=-\varepsilon\left(e_{\alpha}\right)$. Pela parte anterior da prova temos que a condição (i) é atendida. Agora note que

$$
\begin{gathered}
\varepsilon\left(x_{\alpha}\right)=\varepsilon\left(e_{\alpha}\right)-\varepsilon\left(-\varepsilon\left(e_{\alpha}\right)\right)=-f_{\alpha}+e_{\alpha}=x_{\alpha}, \\
\varepsilon\left(y_{\alpha}\right)=\varepsilon\left(i e_{\alpha}\right)+\varepsilon\left(-i \varepsilon\left(e_{\alpha}\right)\right)=i f_{\alpha}+i e_{\alpha}=y_{\alpha},
\end{gathered}
$$

o que implica que a condição (ii) é atendida.

Agora mostramos a ultima parte da proposição. Note que $i h_{\alpha} \in \mathfrak{k}$ já que $\left[x_{\alpha}, y_{\alpha}\right]=2 i h_{\alpha}$. Como $\operatorname{span}_{\mathbb{C}}\left(\left\{x_{\alpha}, y_{\alpha}, i h_{\alpha}: \alpha \in R_{+}\right\}\right)=\mathfrak{g}_{s}=\mathbb{C} \otimes \mathfrak{k}_{s}$ e $\left\{x_{\alpha}, y_{\alpha}, i h_{\alpha}: \alpha \in R_{+}\right\} \subset \mathfrak{k}_{s}$, temos que $\operatorname{span}_{\mathbb{R}}\left(\left\{x_{\alpha}, y_{\alpha}, i h_{\alpha}: \alpha \in R_{+}\right\}\right)=\mathfrak{k}_{s}$.

\subsubsection{Representações de álgebras de Lie compactas}

Seja $W$ uma representação unitária do grupo compacto $K \subset \mathrm{U}(W)$, onde $\mathrm{U}(W)$ é o grupo unitário de $W$. Seja $\mathfrak{k}$ a álgebra de Lie de $K$. Naturalmente $\mathfrak{k} \subset \mathfrak{u}(W)$, onde $\mathfrak{u}(W)$ é o conjunto dos endomorfismos anti-adjuntos de $W$. A complexificação de $\mathfrak{k}$ pode ser incluída em $\mathfrak{g l}(W)$ pela aplicação definida por $z \otimes x \mapsto z x$ para $z \in \mathbb{C}, x \in \mathfrak{k}$. Identificamos $\mathfrak{g}=\mathbb{C} \otimes \mathfrak{k}$ com a sua imagem por essa inclusão. Tome $\mathfrak{t} \subset \mathfrak{k}$ a álgebra de Lie de um toro maximal de $K$ e $\mathfrak{h}=\mathbb{C} \otimes \mathfrak{t}$. Como todo $t \in \mathfrak{t}$ é anti-adjunto e $\mathfrak{t}$ é abeliano, temos que os elementos de $\mathfrak{t}$ podem ser diagonalizados simultaneamente. Como $\mathfrak{h}=\mathbb{C} \otimes \mathfrak{t}$, os elementos de $\mathfrak{h}$ também podem ser diagonalizados simultaneamente, portanto 
$W$ aceita uma decomposição

$$
W=\bigoplus_{\mu \in \Lambda} W_{\mu},
$$

onde $\Lambda \subset \mathfrak{h}^{*}$ é o conjunto de pesos da representações $W$ e $W_{\mu}$ é o espaço peso associado ao peso $\mu$, isso é

$$
W_{\mu}=\{w \in W: h . w=\mu(h) w \forall h \in \mathfrak{h}\} .
$$

Um vetor $w \in W$ é chamado vetor peso se existir um peso $\mu \in \Lambda$ tal que $w \in W_{\mu}$. Nesse caso dizemos que $\mu$ é o peso associado a $w$. A decomposição em espaços peso tem várias propriedades que serão usada no Capítulo 2. Mostramos algumas delas a seguir.

Sejam $\mu_{1}, \mu_{2} \in \Lambda$ dois pesos distintos. Escolhendo um $t \in \mathfrak{t}$ tal que $\mu_{1}(t) \neq \mu_{2}(t)$, temos que $W_{\mu_{1}}$ e $W_{\mu_{2}}$ são autoespaços de $t$ associados a autovalores distintos. Então como $t$ é anti-adjunto, segue que

$$
W_{\mu_{1}} \perp W_{\mu_{2}} .
$$

Ou seja, espaços peso associados a pesos distintos são ortogonais.

Note que para todos $w \in W_{\mu}, e \in \mathfrak{g}_{\alpha}$ e $h \in \mathfrak{h}$ temos que h.e.w $=[h, e] . w+e . h . w=(\alpha(h)+$ $\mu(h)) e . w$ e portanto

$$
\mathfrak{g}_{\alpha} \cdot W_{\mu} \subset W_{\mu+\alpha} .
$$

Como $W$ tem dimensão finita deve haver um vetor de peso maximal, isso é, um vetor peso $w \neq 0$ tal que $e . w=0$ para todo $e \in \mathfrak{n}_{+}=\bigoplus_{\alpha \in R_{+}} \mathfrak{g}_{\alpha}$. O peso $\lambda \in \Lambda$ é chamado maximal se $W_{\lambda}$ contêm algum vetor de peso maximal. A representação $W$ é irredutível se, e somente se, $W$ tiver um único peso maximal $\lambda$ e $\operatorname{dim}_{\mathbb{C}} W_{\lambda}=1$.

Também usaremos o fato que

$$
B\left(\mu, \alpha^{\vee}\right) \in \mathbb{Z}, \forall \mu \in \Lambda, \alpha \in R .
$$

Para provas desses fatos veja [Hum78].

Se $W$ tiver uma estrutura real $\sigma$, então para todo todos $\mu \in \Lambda, w \in W_{\mu}$ e $t \in \mathfrak{t}$, temos que $t . \sigma(w)=\sigma(t . w)=\sigma(\mu(t) w)=\overline{\mu(t)} \sigma(w)$. Como $t$ é anti-adjunto todos os autovalores de $t$ são imaginários puros, então temos que $\overline{\mu(t)}=-\mu(t)$ e portanto

$$
\sigma\left(W_{\mu}\right)=W_{-\mu} .
$$

Então $-\Lambda=\Lambda$ e, para todo $\mu \in \Lambda$, temos que $\operatorname{dim}_{\mathbb{C}}\left(W_{\mu}\right)=\operatorname{dim}_{\mathbb{C}}\left(W_{-\mu}\right)$.

Proposição 1.4.3. Seja $\alpha \in R_{+}$e sejam e e $f_{\alpha}$ como na Proposição 1.4.2. Então para todos $w_{1}, w_{2} \in W$ temos

$$
\left\langle e_{\alpha} \cdot w_{1}, w_{2}\right\rangle=\left\langle w_{1}, f_{\alpha} \cdot w_{2}\right\rangle .
$$

Adicionalmente, para todo $\mu \in \Lambda$ e para todo $w \in W_{\mu}$ temos

$$
\left\|f_{\alpha} \cdot w\right\|^{2}=\left\|e_{\alpha} \cdot w\right\|^{2}+B\left(\mu, \alpha^{\vee}\right)\|w\|^{2} .
$$

Demonstração. Como $e_{\alpha}-f_{\alpha}, i\left(e_{\alpha}+f_{\alpha}\right) \in \mathfrak{k} \subset \mathfrak{u}(W)$ temos que

$$
\begin{aligned}
\left\langle\left(e_{\alpha}-f_{\alpha}\right) \cdot w_{1}, w_{2}\right\rangle & =-\left\langle w_{1},\left(e_{\alpha}-f_{\alpha}\right) \cdot w_{2}\right\rangle, \\
\left\langle i\left(e_{\alpha}+f_{\alpha}\right) \cdot w_{1}, w_{2}\right\rangle & =-\left\langle w_{1}, i\left(e_{\alpha}+f_{\alpha}\right) \cdot w_{2}\right\rangle .
\end{aligned}
$$

Segue de (1.10) que $\left\langle\left(e_{\alpha}+f_{\alpha}\right) \cdot w_{1}, w_{2}\right\rangle=\left\langle w_{1},\left(e_{\alpha}+f_{\alpha}\right) \cdot w_{2}\right\rangle$. A identidade (1.7) é obtida somando essa identidade à identidade (1.9). 
Seja $w \in W_{\mu}$, usando (1.7) temos

$$
\begin{aligned}
\left\langle f_{\alpha} \cdot w, f_{\alpha} \cdot w\right\rangle & =\left\langle w, e_{\alpha} \cdot f_{\alpha} \cdot w\right\rangle \\
& =\left\langle w, f_{\alpha} \cdot e_{\alpha} \cdot w+\left[e_{\alpha}, f_{\alpha}\right] \cdot w\right\rangle \\
& =\left\langle e_{\alpha} \cdot w, e_{\alpha} \cdot w\right\rangle+\left\langle w, h_{\alpha} \cdot w\right\rangle \\
& =\left\|e_{\alpha} \cdot w\right\|^{2}+B\left(\mu, \alpha^{\vee}\right)\|w\|^{2} .
\end{aligned}
$$

Um caso particular importante de (1.8) ocorre quando $w$ é vetor de peso maximal. Note que nesses casos $e_{\alpha} \cdot w=0$ e portanto

$$
\left\|f_{\alpha} \cdot w\right\|^{2}=B\left(\lambda, \alpha^{\vee}\right)\|w\|^{2},
$$

onde $\lambda$ é o peso de $w$. 


\section{Capítulo 2}

\section{Resultados}

Neste capítulo apresentamos e provamos os resultados principais desta dissertação. Aqui vemos uma ação isométrica de um grupo de Lie compacto $K$ em $S^{n}$ como a restrição de uma representação ortogonal de $K \mathrm{em} \mathbb{R}^{n+1}$. Assim como na Subsecção 1.3.2, sempre que calculamos a segunda forma fundamental da órbita de uma representação, estamos calculando a segunda forma fundamental dessa órbita como subvariedade da esfera que a contém. Os casos onde a ação é a restrição de uma representação dada pela realificação de uma representação unitária são particularmente importantes.

Na Secção 2.1 apresentamos o Teorema 2.1.1, que implica que toda representação unitária tem uma órbita a distância um da origem (mais especificamente, a órbita de um vetor de peso maximal) onde as curvaturas principais são majoradas por $4 \sqrt{7}$. Em seguida apresentamos o Teorema 2.1.2, que relaciona a segunda forma fundamental da órbita por um vetor de peso maximal de uma representação unitária de tipo real com a segunda forma fundamental da órbita pela projeção de desse vetor na forma real. Terminamos a Secção 2.1 mostrando o Corolário 2.1.3. Esse Corolário segue facilmente desses dois teoremas, e implica que toda a ação isométrica de um grupo de Lie compacto em uma esfera unitária tem uma órbita com curvaturas principais majoradas por $4 \sqrt{14}$. As Secções 2.2 e 2.3 são dedicadas às provas dos Teoremas 2.1.1 e 2.1.2, respectivamente.

Nesse capítulo, $W$ é uma representação unitária de $K$ e $\langle$,$\rangle é o produto hermitiano em W$. Assim como na Secção 1.4, ké a álgebra de Lie de $K, \mathfrak{t} \subset \mathfrak{k}$ é a álgebra de Lie de um toro maximal de $K$, g é a complexificação de $\mathfrak{k}$ e $\mathfrak{h}$ é a complexificação de $\mathfrak{t}$. Seja $p \in W$, escreveremos $\operatorname{II}_{p}$ para a segunda forma fundamental de $K p$ em $p$ como subvariedade da esfera contendo $p$.

\subsection{Apresentação dos resultados}

Dizemos que uma subálgebra simples $\mathfrak{k}^{\prime} \subset \mathfrak{k}$ é de tipo $A_{n}, B_{n}, C_{n}, D_{n}, E_{6}, E_{7}, E_{8}, F_{4}$ ou $G_{2}$ se $\mathbb{C} \otimes \mathfrak{k}^{\prime}$ for desse mesmo tipo. A subálgebra simples $\mathfrak{k}^{\prime}$ é chamada de clássica se $\mathfrak{k}^{\prime}$ for de tipo $A_{n}, B_{n}, C_{n}$ ou $D_{n}$, caso contrário $\mathfrak{k}^{\prime}$ é chamada de excepcional.

Teorema 2.1.1. Seja $W$ uma representação unitária de um grupo compacto $K$ e sejam $\mathfrak{k}_{i}$ os ideais simples de $\mathfrak{k}(i=1, \cdots, n)$. Tomando $p \in W,\|p\|=1$, um vetor de peso maximal, temos que $\left\|\mathrm{II}_{p}\right\|_{\infty} \leq \max _{i \in\{1, \cdots, n\}} C_{i}$ onde os $C_{i}$ são definidos por:

(i) $C_{i}=\sqrt{6}$ se $\mathfrak{k}_{i}$ for de tipo $G_{2}$;

(ii) $C_{i}=2 \sqrt{3}$ se $\mathfrak{k}_{i}$ for de tipo $A_{n}$;

(iii) $C_{i}=2 \sqrt{6}$ se $\mathfrak{k}_{i}$ for de tipo $B_{n}, C_{n}$ ou $D_{n}$;

(iv) $C_{i}=2 \sqrt{7}$ se $\mathfrak{k}_{i}$ for de tipo $F_{4}$;

(v) $C_{i}=2 \sqrt{10}$ se $\mathfrak{k}_{i}$ for de tipo $E_{6}$;

(vi) $C_{i}=8$ se $\mathfrak{k}_{i}$ for de tipo $E_{7}$;

(vii) $C_{i}=4 \sqrt{7}$ se $\mathfrak{k}_{i}$ for de tipo $E_{8}$.

Como o teorema acima só se aplica às representações ortogonais que são realificações de representações unitárias, precisamos de um outro resultado para ser aplicado a representações ortogonais de tipo real. Esse é o propósito do Teorema abaixo. 
Teorema 2.1.2. Seja $W$ uma representação unitária irredutivel de $K$ de tipo real, $p$ um vetor de peso maximal e $\pi$ a projeção ortogonal na forma real de $W$. Então temos que

$$
\left\|\mathrm{II}_{\pi(p)}\right\|_{\infty} \leq 2\left\|\mathrm{II}_{p}\right\|_{\infty} .
$$

Usando esses dois teoremas, obtemos o corolário abaixo. Como o diâmetro de espaços de órbitas de ações redutíveis é maior ou igual a $\frac{\pi}{2}$, o Corolário 1.2.5 só é útil para ações irredutíveis. Por isso, formulamos o resultado abaixo apenas para representações irredutíveis.

Corolário 2.1.3. Seja $V$ uma representação ortogonal irredutivel do grupo compacto K. Sejam $\mathfrak{k}_{i}$, $i=1, \cdots, n$ os ideais simples da álgebra de Lie de $K$. Sejam $C_{i}, i=1, \cdots, n$ como no Teorema 2.1.1 e tome $C=\max _{i \in\{1, \cdots, n\}} C_{i}$. Então existe $p \in V$ com norma um tal que:

(i) $\left\|\mathrm{II}_{p}\right\|_{\infty} \leq C$, se $V$ for de tipo complexo ou quaterniônico;

(ii) $\left\|\mathrm{II}_{p}\right\|_{\infty} \leq C \sqrt{2}$, se $V$ for de tipo real.

Demonstração. Primeiramente considere o caso onde $V$ é de tipo complexo ou de tipo quaterniônico. Então $V$ é a realificação de uma representação unitária de $K$. Escolha $p \in V$, $\|p\|=1$, um vetor de peso maximal. O fato que $\left\|\mathrm{II}_{p}\right\|_{\infty} \leq C$ segue diretamente do Teorema 2.1.1.

Agora considere o caso onde $V$ é de tipo real. Seja $W$ a complexificação de $V$ e seja $\sigma$ a conjugação complexa de $W$ sobre $V$. Tome $q \in W,\|q\|=\sqrt{2}$, um vetor de peso maximal. Identifique $V$ com o subespaço de $W$ mantido invariante pela estrutura real $\sigma$ e tome $p=\pi(q)$, onde $\pi=\frac{1}{2}(I d+\sigma)$ é a projeção ortogonal em $V$. Como o peso associado a $q$ é diferente de zero, segue de (1.6) que $\sigma(q) \perp q$, e portanto $\|p\|=1$. Usando o Teorema 2.1.2, (1.5) e o Teorema 2.1.1 temos que

$$
\left\|\mathrm{II}_{p}\right\|_{\infty} \leq 2\left\|\mathrm{II}_{q}\right\|_{\infty}=\sqrt{2}\left\|\mathrm{II}_{\frac{q}{\sqrt{2}}}\right\|_{\infty} \leq C \sqrt{2} .
$$

Segue do Corolário 2.1.3 que toda ação isométrica de um grupo de Lie compacto $K$ em uma esfera unitária $S^{n}$ tem uma órbita com curvaturas principais majoradas por $4 \sqrt{14}$. Para mostrar esse fato basta tomar $V \subset \mathbb{R}^{n+1}$ uma subrepresentação irredutível de $K$ e aplicar o Corolário 2.1.3 a $V$. Note que a norma do supremo da segunda forma fundamental de $K p$ não muda se considerarmos $K p$ como subvariedade de $S^{n} \cap V$ ou de $S^{n}$, já que $S^{n} \cap V$ é totalmente geodésica em $S^{n}$.

\subsection{Curvatura de órbitas de representações unitárias}

Seja $W$ uma representação unitária do grupo de Lie compacto $K$. Consideraremos $W$ como um espaço vetorial real com produto simétrico $\langle,\rangle_{\mathbb{R}}$, a menos que seja explicitado o contrário. Seja $p \in W,\|p\|=1 \mathrm{um}$ vetor de peso maximal de $W$ e seja $\lambda$ o peso associado a $p$. Como o caso onde a órbita $K p$ é discreta é trivial, assumimos que $\lambda \neq 0$. Para todo $\alpha \in R_{+}$tomamos $e_{\alpha}, f_{\alpha}, x_{\alpha}$ e $y_{\alpha}$ como na Proposição 1.4.2. Como consideramos a segunda forma fundamental apenas em $p$, escrevemos II para II $_{p}$.

Começamos essa secção mostrando que

$$
\mathcal{B}=\left\{f_{\alpha} \cdot p, i f_{\alpha} \cdot p: \alpha \in R_{+},\left\|f_{\alpha} \cdot p\right\|>0\right\} \cup\{i p\}
$$

é uma base ortogonal de k $p$ e estudando II nessa base. Dentre os fatos mais importantes que mostraremos estão estimativas para $\left\|\operatorname{II}\left(\frac{f_{\alpha} \cdot p}{\left\|f_{\alpha} \cdot p\right\|}, \frac{f_{\beta} \cdot p}{\left\|f_{\beta} \cdot p\right\|}\right)\right\|$ que dependem apenas do ângulo entre $\alpha$ e $\beta$, e o fato que para estimar $\|\mathrm{II}\|_{\infty}$ basta considerar os casos onde $K$ é simples.

Nas Subsecção 2.2.1 usamos os fatos aprendidos sobre a segunda forma fundamental na base $\mathcal{B}$ para propor um método para encontrar estimativas de $\|\mathrm{II}\|_{\infty}$ que depende apenas do sistema de raízes $R$. Aplicamos esse método aos sistemas de raízes clássicos e, organizando as contas usando matrizes de números não negativos, mostramos que $\|\mathrm{II}\|_{\infty} \leq 2 \sqrt{6}$ se $\mathfrak{k}$ for uma álgebra de Lie clássica. 
Devido a complicações nos sistemas de raízes excepcionais, não é fácil aplicar o mesmo método para esses sistemas de raízes. Na Subsecção 2.2.2 propomos um método mais grosseiro para estimar $\|\mathrm{II}\|_{\infty}$ que também depende apenas de $R$. Esse método também pode ser usado para álgebras de Lie clássicas, mas, como a estimativa dada por ele tende ao infinto com o posto do sistema de raízes, restringimos seu uso às álgebras de Lie excepcionais. Esse método pode ser facilmente implementado em qualquer sistema computacional algébrico com suporte para sistemas de raízes. Nós usamos uma implementação em SageMath para calcular estimativas para $\|\mathrm{II}\|_{\infty}$ quando $\mathfrak{k}$ é uma álgebra de Lie excepcional.

Proposição 2.2.1. Seja $p \in W$ um vetor de peso maximal. Então

$$
T_{p} K p=\mathfrak{k} \cdot p=\mathfrak{n}_{-} . p \oplus \mathbb{R} i p,
$$

onde $\mathfrak{n}_{-}=\bigoplus_{\alpha \in R_{+}} \mathfrak{g}_{-\alpha}$.

Demonstração. A primeira equação segue diretamente de (1.3). Para mostrar a segunda equação usamos a Proposição 1.4.2. Como $\left\{x_{\alpha}, y_{\alpha}: \alpha \in R_{+}\right\} \cup \mathfrak{t}$ gera $\mathfrak{k}$, temos que

$$
\mathfrak{k} \cdot p=\left(\sum_{\alpha \in R_{+}} \mathbb{R} x_{\alpha} \cdot p+\mathbb{R} y_{\alpha} \cdot p\right)+\mathfrak{t} \cdot p .
$$

Como $p$ é vetor de peso maximal, para todo $\alpha \in R_{+}$temos que

$$
x_{\alpha} \cdot p=\left(e_{\alpha}-f_{\alpha}\right) \cdot p=-f_{\alpha} \cdot p \quad \text { e } \quad y_{\alpha} \cdot p=i\left(e_{\alpha}+f_{\alpha}\right) \cdot p=i f_{\alpha} \cdot p .
$$

Portanto $\mathbb{R} x_{\alpha} \cdot p+\mathbb{R} y_{\alpha} \cdot p=\mathfrak{g}_{-\alpha} \cdot p$.

Como todo $t \in \mathfrak{t}$ é anti-adjunto, todos os autovalores de $t$ são imaginários puros. Portanto $\mathfrak{t} . p=\lambda(\mathfrak{t}) p=\mathbb{R} i p$.

Como $\nu_{p} K p=(\mathfrak{k} p \oplus \mathbb{R} p)^{\perp}$ e $p$ é vetor de peso maximal, temos que

$$
\nu_{p} K p=(\mathfrak{g} \cdot p)^{\perp},
$$

onde $(\mathfrak{g} \cdot p)^{\perp}$ é o complemento ortogonal de $\mathfrak{g} \cdot p$ em $W$. Seja $(\cdot)^{\perp}$ a projeção ortogonal de $W$ em $(\mathfrak{g} \cdot p)^{\perp}$. Agora vamos mostrar que $(\cdot)^{\perp}$ é $\mathbb{C}$-linear e preserva os espaços peso. Como $\mathcal{B} \cup\{p\}$ é uma base ortogonal de $\mathfrak{g} \cdot p$, para qualquer $w \in W$ temos que

$$
\begin{aligned}
(w)^{\perp} & =w-\left(\langle w, p\rangle_{\mathbb{R}} p+\langle w, i p\rangle_{\mathbb{R}} i p+\sum_{f_{\alpha} \cdot p \in \mathcal{B}}\left\langle w, f_{\alpha} \cdot p\right\rangle_{\mathbb{R}} \frac{f_{\alpha} \cdot p}{\left\|f_{\alpha} \cdot p\right\|^{2}}+\left\langle w, i f_{\alpha} \cdot p\right\rangle_{\mathbb{R}} \frac{i f_{\alpha} \cdot p}{\left\|i f_{\alpha} \cdot p\right\|^{2}}\right) \\
& =w-\left(\langle w, p\rangle p+\sum_{f_{\alpha} \cdot p \in \mathcal{B}}\left\langle w, f_{\alpha} \cdot p\right\rangle \frac{f_{\alpha} \cdot p}{\left\|f_{\alpha} \cdot p\right\|^{2}}\right) .
\end{aligned}
$$

Como $\langle$,$\rangle é hermitiano, segue que (\cdot)^{\perp}$ é $\mathbb{C}$-linear. Note que se $w$ é um vetor peso com peso associado $\mu$ temos que

$$
(w)^{\perp}= \begin{cases}w-\langle w, p\rangle p & \text { se } \mu=\lambda, \\ w-\left\langle w, f_{\alpha} \cdot p\right\rangle \frac{f_{\alpha} \cdot p}{\left\|f_{\alpha} \cdot p\right\|^{2}} & \text { se } \mu=\lambda-\alpha \text { para algum } \alpha \in R_{+} \operatorname{com}\left\|f_{\alpha} \cdot p\right\|>0, \\ w & \text { caso contrário. }\end{cases}
$$

Então $(w)^{\perp} \in W_{\mu}$, ou seja, $(\cdot)^{\perp}$ preserva os espaços peso.

Voltamos nossa atenção para a segunda forma fundamental em $p$. Sejam $\alpha, \beta \in R_{+}$quaisquer. 
Temos que

$$
\begin{aligned}
\operatorname{II}\left(f_{\alpha} \cdot p, f_{\beta} \cdot p\right) & =\operatorname{II}\left(-x_{\alpha} \cdot p,-x_{\beta} \cdot p\right) \\
& =\left(x_{\alpha} \cdot x_{\beta} \cdot p\right)^{\perp} \\
& =\left(f_{\alpha} \cdot f_{\beta} \cdot p\right)^{\perp}-\left(e_{\alpha} \cdot f_{\beta} \cdot p\right)^{\perp} \\
& =\left(f_{\alpha} \cdot f_{\beta} \cdot p\right)^{\perp}-\left(\left[e_{\alpha}, f_{\beta}\right] \cdot p\right)^{\perp} \\
& =\left(f_{\alpha} \cdot f_{\beta} \cdot p\right)^{\perp} .
\end{aligned}
$$

Onde a ultima igualdade segue do fato que $\left[e_{\alpha}, f_{\beta}\right] \cdot p \in \mathfrak{g} \cdot p$.

Similarmente, é possível mostrar que $\operatorname{II}\left(i f_{\alpha} \cdot p, f_{\beta} \cdot p\right)=\left(i f_{\alpha} \cdot f_{\beta} \cdot p\right)^{\perp}$ e $\operatorname{II}\left(i f_{\alpha} \cdot p, i f_{\beta} \cdot p\right)=-\left(f_{\alpha} \cdot f_{\beta} \cdot p\right)^{\perp}$. Usando esses fatos e as observações sobre $(\cdot)^{\perp}$ feitas acima, temos que II $\left.\right|_{\mathfrak{n}_{-} . p \times \mathfrak{n}_{-} . p}$ é $\mathbb{C}$-bilinear e

$$
\mathrm{II}\left(f_{\alpha} \cdot p, f_{\beta} \cdot p\right) \in W_{\lambda-\alpha-\beta} .
$$

Seja $x \in \mathfrak{k}$ qualquer. Então $\operatorname{II}(i p, x . p)=(i x . p)^{\perp}$. Como $i x \in \mathfrak{g}$ temos que

$$
\mathrm{II}(i p, x \cdot p)=0 .
$$

Logo, pela Proposição 1.2.3, para estimar $\|\mathrm{II}\|_{\infty}$ basta estimar $\|\mathrm{II}(f . p, f . p)\|$ onde $f \in \mathfrak{n}_{-}$é tal que $\|f \cdot p\|=1$.

Sejam $f_{\alpha} \cdot p, f_{\beta} \cdot p \in \mathcal{B}$. O resultado a seguir mostra que é possível estimar $\left\|\mathrm{II}\left(\frac{f_{\alpha} \cdot p}{\left\|f_{\alpha} \cdot p\right\|}, \frac{f_{\beta} \cdot p}{\left\|f_{\beta} \cdot p\right\|}\right)\right\|$ independentemente de $\lambda$.

Proposição 2.2.2. Sejam $\alpha, \beta \in R_{+}$, então

$$
\left\|\operatorname{II}\left(f_{\alpha} \cdot p, f_{\beta} \cdot p\right)\right\| \leq \begin{cases}\sqrt{2}\left\|f_{\alpha} \cdot p\right\|\left\|f_{\beta} \cdot p\right\| & \text { se } \alpha=\beta \text { ou } B(\alpha, \beta)<0, \\ \left\|f_{\alpha} \cdot p\right\|\left\|f_{\beta} \cdot p\right\| & \text { caso contrário. }\end{cases}
$$

Demonstração. Note que se $\left\|f_{\alpha} \cdot p\right\|=0$ ou $\left\|f_{\beta} \cdot p\right\|=0$ as inequações acima são triviais. Portanto assumiremos que $\left\|f_{\alpha} \cdot p\right\|,\left\|f_{\beta} \cdot p\right\|>0$. Primeiramente note que como $p$ é vetor de peso maximal e tem norma um segue de (1.8) que

$$
\left\|f_{\alpha} \cdot p\right\|^{2}=B\left(\lambda, \alpha^{\vee}\right) \quad \text { e } \quad\left\|f_{\beta} \cdot p\right\|^{2}=B\left(\lambda, \beta^{\vee}\right) .
$$

Usando essas equações temos que

$$
\begin{array}{rlr}
\left\|\operatorname{II}\left(f_{\alpha} \cdot p, f_{\beta} \cdot p\right)\right\|^{2} & =\left\|\left(f_{\alpha} \cdot f_{\beta} \cdot p\right)^{\perp}\right\|^{2} & \\
& \leq\left\|f_{\alpha} \cdot f_{\beta} \cdot p\right\|^{2} & \\
& =\left\|e_{\alpha} \cdot f_{\beta} \cdot p\right\|^{2}+B\left(\lambda-\beta, \alpha^{\vee}\right)\left\|f_{\beta} \cdot p\right\|^{2} & \text { (por (2.2)) } \\
& =\left\|\left[e_{\alpha}, f_{\beta}\right] \cdot p\right\|^{2}+B\left(\lambda, \alpha^{\vee}\right)\left\|f_{\beta} \cdot p\right\|^{2}-B\left(\beta, \alpha^{\vee}\right)\left\|f_{\beta} \cdot p\right\|^{2} & \text { (como } p \text { é de peso maximal) } \\
& =\left\|\left[e_{\alpha}, f_{\beta}\right] \cdot p\right\|^{2}+\left\|f_{\alpha} \cdot p\right\|^{2}\left\|f_{\beta} \cdot p\right\|^{2}-B\left(\beta, \alpha^{\vee}\right)\left\|f_{\beta} \cdot p\right\|^{2} . &
\end{array}
$$

Analisamos essa desigualdade em quatro casos distintos.

Caso 1: $\alpha=\beta$

Como $B\left(\alpha, \alpha^{\vee}\right)=2$, temos que

$$
\begin{aligned}
\left\|\mathrm{II}\left(f_{\alpha} \cdot p, f_{\alpha} \cdot p\right)\right\|^{2} & \leq\left\|h_{\alpha} \cdot p\right\|^{2}+\left\|f_{\alpha} \cdot p\right\|^{4}-2\left\|f_{\alpha} \cdot p\right\|^{2} \\
& \leq\|B(\lambda, \alpha) p\|^{2}+\left\|f_{\alpha} \cdot p\right\|^{4} \\
& =2\left\|f_{\alpha} \cdot p\right\|^{4} .
\end{aligned}
$$


Caso 2: $\alpha \neq \beta$ e $B(\alpha, \beta) \geq 0$

Podemos assumir sem perda de generalidade que $\beta-\alpha \notin R_{+}$já que se $\beta-\alpha \in R_{+}$, permutando $\alpha$ e $\beta$, temos que $\beta-\alpha \notin R_{+}$e, por simetria, $\operatorname{II}\left(f_{\alpha} \cdot p, f_{\beta} \cdot p\right)$ permanece igual. Então temos que $\left\|\left[e_{\alpha}, f_{\beta}\right] \cdot p\right\|=0$. Usando o fato que $B\left(\beta, \alpha^{\vee}\right) \geq 0$ temos

$$
\left\|\operatorname{II}\left(f_{\alpha} \cdot p, f_{\beta} \cdot p\right)\right\|^{2} \leq\left\|f_{\alpha} \cdot p\right\|^{2}\left\|f_{\beta} \cdot p\right\|^{2}-B\left(\beta, \alpha^{\vee}\right)\left\|f_{\beta} \cdot p\right\|^{2} \leq\left\|f_{\alpha} \cdot p\right\|^{2}\left\|f_{\beta} \cdot p\right\|^{2} .
$$

Caso 3: $B(\alpha, \beta)<0$ e $\|\beta\|=\|\alpha\|$

Assim como no Caso 2, podemos assumir sem perda de generalidade que $\beta-\alpha \notin R_{+}$. Segue que $\left\|\left[e_{\alpha}, f_{\beta}\right] . p\right\|=0$. Pela tabela A.1 temos que $B\left(\beta, \alpha^{\vee}\right)=-1$, e portanto

$$
\left\|\operatorname{II}\left(f_{\alpha} \cdot p, f_{\beta} \cdot p\right)\right\|^{2} \leq\left\|f_{\alpha} \cdot p\right\|^{2}\left\|f_{\beta} \cdot p\right\|^{2}+\left\|f_{\beta} \cdot p\right\|^{2} .
$$

Como $W$ é uma representação de dimensão finita de $\mathfrak{g}$, temos que $B\left(\lambda, \alpha^{\vee}\right)$ é um inteiro. Como assumimos que $\left\|f_{\alpha} \cdot p\right\|$ é positivo, devemos ter que $\left\|f_{\alpha} \cdot p\right\|^{2}=B\left(\lambda, \alpha^{\vee}\right) \geq 1$ e portanto $\left\|f_{\beta} \cdot p\right\|^{2} \leq$ $\left\|f_{\alpha} \cdot p\right\|^{2}\left\|f_{\beta} \cdot p\right\|^{2}$. Então

$$
\left\|\operatorname{II}\left(f_{\alpha} \cdot p, f_{\beta} \cdot p\right)\right\|^{2} \leq 2\left\|f_{\alpha} \cdot p\right\|^{2}\left\|f_{\beta} \cdot p\right\|^{2} .
$$

Caso 4: $B(\alpha, \beta)<0$ e $\|\beta\| \neq\|\alpha\|$

Permutando $\alpha$ e $\beta$ podemos assumir sem perda de generalidade que $\alpha$ é longa e $\beta$ é curta, e portanto pela tabela A.1 temos que $B\left(\beta, \alpha^{\vee}\right)=-1$. Como $B(\alpha, \beta)<0$, temos que $\|\beta-\alpha\|>$ $\|\alpha\|$, e então $\|\beta-\alpha\|$ não pode ser uma raiz, já que caso contrário o sistema de raízes irredutível $(\mathbb{Z} \alpha+\mathbb{Z} \beta) \cap R$ teria raízes de três comprimentos diferentes. Segue que $\left\|\left[e_{\alpha}, f_{\beta}\right] \cdot p\right\|=0$. Repetindo a mesma argumentação usada no Caso 3 temos que

$$
\left\|\mathrm{II}\left(f_{\alpha} \cdot p, f_{\beta} \cdot p\right)\right\|^{2} \leq 2\left\|f_{\alpha} \cdot p\right\|^{2}\left\|f_{\beta} \cdot p\right\|^{2} .
$$

É fácil ver que usando o resultado acima podemos estimar $\|\mathrm{II}\|_{\infty}$ usando apenas informações sobre o sistema de raízes $R$ de $\mathfrak{g}$. O resultado seguinte nos diz que basta calcular essa estimativa separadamente nas componentes irredutíveis de $R$.

Proposição 2.2.3. Seja $\mathfrak{k}=\bigoplus_{i=1}^{n} \mathfrak{k}_{i} \oplus \mathfrak{a}$ a decomposição de $\mathfrak{k}$ em seus ideais simples $\mathfrak{k}_{i}$ e em sua componente abeliana $\mathfrak{a}$. Seja $K_{i}=\operatorname{Exp}\left(\mathfrak{k}_{i}\right)$ e $\mathrm{II}_{i}$ a segunda forma fundamental de $K_{i} p$. Seja $C \geq \sqrt{2}$ tal que $\left\|\mathrm{II}_{i}\right\|_{\infty} \leq C$ para $i=1, \cdots, n$. Então $\|\mathrm{II}\|_{\infty} \leq C$.

Demonstração. Basta mostrar que para todo $f \in \mathfrak{n}_{-}$temos que $\|\operatorname{II}(f . p, f . p)\| \leq C\|f . p\|^{2}$. Seja $f \in \mathfrak{n}_{-}$, seja $\mathfrak{g}_{i}$ a complexificação de $\mathfrak{k}_{i}$ e seja $R_{+}^{i} \subset R_{+}$o conjunto das raízes positivas de $\mathfrak{g}_{i}$. Seja $z_{\alpha} \in \mathbb{C}$ para $\alpha \in R_{+}$tal que $f=\sum_{\alpha \in R_{+}} z_{\alpha} f_{\alpha}$, e seja $f_{i}=\sum_{\alpha \in R_{+}^{i}} z_{\alpha} f_{\alpha}$.

Começamos mostrando que $\left\|\mathrm{II}\left(f_{i} \cdot p, f_{j} . p\right)\right\| \leq\left\|f_{i} \cdot p\right\|\left\|f_{j} . p\right\|$ para $i \neq j$. Note que se $(\alpha, \beta),\left(\alpha^{\prime}, \beta^{\prime}\right) \in$ $R_{+}^{i} \times R_{+}^{j}$ são tais que $(\alpha, \beta) \neq\left(\alpha^{\prime}, \beta^{\prime}\right)$, então $\alpha+\beta \neq \alpha^{\prime}+\beta^{\prime}$ e portanto $\operatorname{II}\left(f_{\alpha} \cdot p, f_{\beta} \cdot p\right) \perp \operatorname{II}\left(f_{\alpha^{\prime}} \cdot p, f_{\beta^{\prime}} \cdot p\right)$. 
Usando esse fato e usando que $R_{+}^{i} \perp R_{+}^{j}$, temos

$$
\begin{aligned}
\left\|\mathrm{II}\left(f_{i} \cdot p, f_{j} \cdot p\right)\right\|^{2} & =\left\|\sum_{(\alpha, \beta) \in R_{+}^{i} \times R_{+}^{j}} z_{\alpha} z_{\beta} \mathrm{II}\left(f_{\alpha} \cdot p, f_{\beta} \cdot p\right)\right\|^{2} \\
& =\sum_{(\alpha, \beta) \in R_{+}^{i} \times R_{+}^{j}}\left\|z_{\alpha} z_{\beta} \mathrm{II}\left(f_{\alpha} \cdot p, f_{\beta} \cdot p\right)\right\|^{2} \\
& \leq \sum_{(\alpha, \beta) \in R_{+}^{i} \times R_{+}^{j}}\left\|z_{\alpha} f_{\alpha} \cdot p\right\|^{2}\left\|z_{\beta} f_{\beta} \cdot p\right\|^{2} \\
& =\left(\sum_{\alpha \in R_{+}^{i}}\left\|z_{\alpha} f_{\alpha} \cdot p\right\|^{2}\right)\left(\sum_{\beta \in R_{+}^{j}}\left\|z_{\beta} f_{\beta} \cdot p\right\|^{2}\right) \\
& =\left\|f_{i} \cdot p\right\|^{2}\left\|f_{j} \cdot p\right\|^{2} .
\end{aligned}
$$

Note que se $i, j, k, l \in\{1, \cdots, n\}$ são tais que $\{i, j\} \neq\{k, l\}$, então $\operatorname{II}\left(f_{i} \cdot p, f_{j} \cdot p\right) \perp \operatorname{II}\left(f_{k} \cdot p, f_{l} \cdot p\right)$. Usando esse fato e a desigualdade acima temos que

$$
\begin{aligned}
\|\mathrm{II}(f \cdot p, f \cdot p)\|^{2} & =\left\|\sum_{i, j} \operatorname{II}\left(f_{i} \cdot p, f_{j} \cdot p\right)\right\|^{2} \\
& =\left\|\sum_{i<j} 2 \operatorname{II}\left(f_{i} \cdot p, f_{j} \cdot p\right)+\sum_{i} \operatorname{II}\left(f_{i} \cdot p, f_{i} \cdot p\right)\right\|^{2} \\
& =\sum_{i<j}\left\|2 \operatorname{II}\left(f_{i} \cdot p, f_{j} \cdot p\right)\right\|^{2}+\sum_{i}\left\|\operatorname{II}\left(f_{i} \cdot p, f_{i} \cdot p\right)\right\|^{2} \\
& \leq 4 \sum_{i<j}\left\|f_{i} \cdot p\right\|^{2}\left\|f_{j} \cdot p\right\|^{2}+\sum_{i}\left\|\operatorname{II}\left(f_{i} \cdot p, f_{i} \cdot p\right)\right\|^{2}
\end{aligned}
$$

Como $\operatorname{II}_{i}\left(f_{i} \cdot p, f_{i} \cdot p\right)=\operatorname{II}\left(f_{i} \cdot p, f_{i} \cdot p\right)$, temos que $\left\|\mathrm{II}\left(f_{i} \cdot p, f_{i} \cdot p\right)\right\| \leq C\left\|f_{i} \cdot p\right\|^{2}$ e portanto

$$
\begin{aligned}
\|\operatorname{II}(f . p, f . p)\|^{2} & \leq 4 \sum_{i<j}\left\|f_{i} \cdot p\right\|^{2}\left\|f_{j} \cdot p\right\|^{2}+C^{2} \sum_{i}\left\|f_{i} \cdot p\right\|^{4} \\
& \left.\leq C^{2}\left(\sum_{i<j} 2\left\|f_{i} \cdot p\right\|^{2}\left\|f_{j} \cdot p\right\|^{2}+\sum_{i}\left\|f_{i} \cdot p\right\|^{4}\right) \quad \quad \text { (já que } C^{2} \geq 2\right) \\
& =C^{2}\|f \cdot p\|^{4} .
\end{aligned}
$$

Na Subsecção 2.2.1 mostraremos:

Proposição 2.2.4. Se ke é uma álgebra de Lie clássica, então

(i) $\|\mathrm{II}\|_{\infty} \leq 2 \sqrt{3}$ se $\mathfrak{k}$ for de tipo $A_{n}$;

(ii) $\|\mathrm{II}\|_{\infty} \leq 2 \sqrt{6}$ se $\mathfrak{k}$ for de tipo $B_{n}, C_{n}$ ou $D_{n}$.

E na Subsecção 2.2.2 mostraremos:

Proposição 2.2.5. Se ké uma álgebra de Lie excepcional, então

(i) $\|\mathrm{II}\|_{\infty} \leq \sqrt{6}$ se ḱ for de tipo $G_{2}$;

(ii) $\|\mathrm{II}\|_{\infty} \leq 2 \sqrt{7}$ se $\mathfrak{k}$ for de tipo $F_{4}$;

(iii) $\|\mathrm{II}\|_{\infty} \leq 2 \sqrt{10}$ se $\mathfrak{k}$ for de tipo $E_{6}$; 
(iv) $\|\mathrm{II}\|_{\infty} \leq 8$ se $\mathfrak{k}$ for de tipo $E_{7}$;

(v) $\|\mathrm{II}\|_{\infty} \leq 4 \sqrt{7}$ se $\mathfrak{k}$ for de tipo $E_{8}$.

\section{Prova do Teorema 2.1.1}

Sejam $\mathfrak{k}_{i}, K_{i}, \mathrm{II}_{i}$ e $n$ como na Proposição 2.2.3. Para $i \in\{1, \cdots, n\}$ defina

(i) $C_{i}=\sqrt{6}$ se $\mathfrak{k}_{i}$ for de tipo $G_{2}$;

(ii) $C_{i}=2 \sqrt{3}$ se $\mathfrak{k}_{i}$ for de tipo $A_{n}$;

(iii) $C_{i}=2 \sqrt{6}$ se $\mathfrak{k}_{i}$ for de tipo $B_{n}, C_{n}$ ou $D_{n}$;

(iv) $C_{i}=2 \sqrt{7}$ se $\mathfrak{k}_{i}$ for de tipo $F_{4}$;

(v) $C_{i}=2 \sqrt{10}$ se $\mathfrak{k}_{i}$ for de tipo $E_{6}$;

(vi) $C_{i}=8$ se $\mathfrak{k}_{i}$ for de tipo $E_{7}$;

(vii) $C_{i}=4 \sqrt{7}$ se $\mathfrak{k}_{i}$ for de tipo $E_{8}$.

Seja $C=\max _{i \in\{1, \cdots, n\}} C_{i}$. Então $\left\|\mathrm{II}_{i}\right\|_{\infty} \leq C$ para $i=1, \cdots, n$ e, da Proposição 2.2.3, temos que $\|\mathrm{II}\|_{\infty} \leq C$.

\subsubsection{Estimativas para $\mathfrak{k}$ clássica}

Começamos desenvolvendo uma estimativa que é comum a todos os casos. Pela Proposição 1.2.3 temos que para mostrar que $\|\mathrm{II}\|_{\infty} \leq C$ basta mostrar que $\|\mathrm{II}(f . p, f . p)\| \leq C$ para qualquer $f \in \mathfrak{n}_{-}$ tal que $\|f . p\|=1$. Escrevendo $f$ na base formada pelos $f_{\alpha}$ 's temos

$$
f=\sum_{\alpha \in R_{+}} z_{\alpha} f_{\alpha}
$$

para $z_{\alpha} \in \mathbb{C}$. Defina $r_{\alpha}=\left\|z_{\alpha} f_{\alpha} \cdot p\right\|$ para $\alpha \in R_{+}$e note que

$$
\sum_{\alpha \in R_{+}} r_{\alpha}^{2}=\sum_{\alpha \in R_{+}}\left\|z_{\alpha} f_{\alpha} \cdot p\right\|^{2}=\|f \cdot p\|^{2}=1 .
$$

Por (2.3) temos que

$$
\left\|z_{\alpha} z_{\beta} \mathrm{II}\left(f_{\alpha} \cdot p, f_{\beta} \cdot p\right)\right\| \leq m(\alpha, \beta) r_{\alpha} r_{\beta}
$$

onde

$$
m(\alpha, \beta)= \begin{cases}\sqrt{2} & \text { se } B(\alpha, \beta)<0 \text { ou } \alpha=\beta \\ 1 & \text { caso contrário. }\end{cases}
$$

Note que

$$
\|\mathrm{II}(f . p, f \cdot p)\|^{2}=\left\|\sum_{\gamma \in 2 R_{+}} \sum_{\substack{\alpha, \beta \\ \alpha+\beta=\gamma}} z_{\alpha} z_{\beta} \mathrm{II}\left(f_{\alpha} \cdot p, f_{\beta} \cdot p\right)\right\|^{2},
$$

onde $2 R_{+}=\left\{\alpha+\beta \mid \alpha, \beta \in R_{+}\right\}$. Como $\operatorname{II}\left(f_{\alpha} \cdot p, f_{\beta} \cdot p\right)=\left(f_{\alpha} \cdot f_{\beta} \cdot p\right)^{\perp} \in W_{\lambda-\alpha-\beta}$, temos que se $\alpha, \beta, \alpha^{\prime}, \beta^{\prime} \in R_{+}$são tais que $\alpha+\beta \neq \alpha^{\prime}+\beta^{\prime}$ então $\operatorname{II}\left(f_{\alpha} \cdot p, f_{\beta} \cdot p\right) \perp \operatorname{II}\left(f_{\alpha^{\prime}} \cdot p, f_{\beta^{\prime}} \cdot p\right)$. Usando esse fato 
temos que

$$
\begin{aligned}
\|\mathrm{II}(f . p, f \cdot p)\|^{2} & =\sum_{\gamma \in 2 R_{+}}\left\|\sum_{\substack{\alpha, \beta \\
\alpha+\beta=\gamma}} z_{\alpha} z_{\beta} \mathrm{II}\left(f_{\alpha} \cdot p, f_{\beta} \cdot p\right)\right\|^{2} \\
& \leq \sum_{\gamma \in 2 R_{+}}\left(\sum_{\substack{\alpha, \beta \\
\alpha+\beta=\gamma}}\left\|z_{\alpha} z_{\beta} \mathrm{II}\left(f_{\alpha} \cdot p, f_{\beta} \cdot p\right)\right\|\right)^{2} \\
& \leq \sum_{\gamma \in 2 R_{+}}\left(\sum_{\substack{\alpha, \beta \\
\alpha+\beta=\gamma}} m(\alpha, \beta) r_{\alpha} r_{\beta}\right)^{2}
\end{aligned}
$$

Note que

$$
2-\sum_{\gamma \in 2 R_{+}} 2 \sum_{\substack{\alpha, \beta \\ \alpha+\beta=\gamma}} r_{\alpha}^{2} r_{\beta}^{2}=2-2 \sum_{\alpha, \beta \in R_{+}} r_{\alpha}^{2} r_{\beta}^{2}=2-2\|f \cdot p\|^{4}=0 .
$$

Então

$$
\|\mathrm{II}(f . p, f . p)\|^{2} \leq 2+\sum_{\gamma \in 2 R_{+}}\left[\left(\sum_{\substack{\alpha, \beta \\ \alpha+\beta=\gamma}} m(\alpha, \beta) r_{\alpha} r_{\beta}\right)^{2}-2 \sum_{\substack{\alpha, \beta \\ \alpha+\beta=\gamma}} r_{\alpha}^{2} r_{\beta}^{2}\right]
$$

Denotamos os termos entre colchetes por $S_{\gamma}$. Iremos estimar os $S_{\gamma}$ em função dos $r_{\alpha}$ para todo $\gamma \in 2 R_{+}$com $R$ clássico. Para isso é essencial entender as decomposições dos $\gamma \in 2 R_{+}$como somas de raízes positivas, o que será feito caso a caso. Mas antes mostramos um resultado mais geral.

Proposição 2.2.6. Seja $\gamma \in 2 R_{+}$. Então $S_{\gamma} \geq 0$. Se $S_{\gamma}>0$, então existem mais de dois $(\alpha, \beta) \in$ $R_{+} \times R_{+}$tais que $\alpha+\beta=\gamma$ ou $\gamma$ é a soma de duas raizes positivas que formam um ângulo obtuso entre si.

Demonstração. Primeiramente vamos mostrar que $S_{\gamma} \geq 0$. Seja $D_{\gamma}=\left\{(\alpha, \beta) \in R_{+} \times R_{+} \mid \alpha+\beta=\right.$ $\gamma\}$. Note que se $(\alpha, \beta) \in D_{\gamma}$, então $(\beta, \alpha)$. Seja $\left\{\left(\alpha_{i}, \beta_{i}\right) \mid i=1, \cdots, n\right\}$ um subconjunto de $D_{\gamma}$ obtido escolhendo exatamente um elemento de cada $\{(\alpha, \beta),(\beta, \alpha)\} \subset D_{\gamma}$. Então, como $m(\alpha, \beta) \geq 1$ e $r_{\alpha} \geq 0$ para todo o $\alpha, \beta \in R_{+}$, temos que

$$
\begin{aligned}
S_{\gamma} & =\left(2 \sum_{\substack{i=1, \cdots n \\
\alpha_{i} \neq \beta_{i}}} m\left(\alpha_{i}, \beta_{i}\right) r_{\alpha_{i}} r_{\beta_{i}}+\sum_{\substack{i=1, \cdots n \\
\alpha_{i}=\beta_{i}}} m\left(\alpha_{i}, \alpha_{i}\right) r_{\alpha_{i}}^{2}\right)^{2}-4 \sum_{\substack{i=1, \cdots n \\
\alpha_{i} \neq \beta_{i}}} r_{\alpha_{i}}^{2} r_{\beta_{i}}^{2}-2 \sum_{\substack{i=1, \cdots n \\
\alpha_{i}=\beta_{i}}} r_{\alpha_{i}}^{4} \\
& \geq\left(2 \sum_{\substack{i=1, \cdots n \\
\alpha_{i} \neq \beta_{i}}} r_{\alpha_{i}} r_{\beta_{i}}+\sqrt{2} \sum_{\substack{i=1, \cdots n \\
\alpha_{i}=\beta_{i}}} r_{\alpha_{i}}^{2}\right)^{2}-4 \sum_{\substack{i=1, \cdots n \\
\alpha_{i} \neq \beta_{i}}} r_{\alpha_{i}}^{2} r_{\beta_{i}}^{2}-2 \sum_{\substack{i=1, \cdots n \\
\alpha_{i}=\beta_{i}}} r_{\alpha_{i}}^{4} \\
& \geq 4\left(\sum_{\substack{i=1, \cdots n \\
\alpha_{i} \neq \beta_{i}}} r_{\alpha_{i}} r_{\beta_{i}}\right)^{2}-4 \sum_{\substack{i=1, \cdots n \\
\alpha_{i} \neq \beta_{i}}} r_{\alpha_{i}}^{2} r_{\beta_{i}}^{2}+2\left(\sum_{\substack{i=1, \cdots n \\
\alpha_{i}=\beta_{i}}} r_{\alpha_{i}}^{2}-2 \sum_{\substack{i=1, \cdots n \\
\alpha_{i}=\beta_{i}}} r_{\alpha_{i}}^{4}\right. \\
& \geq 0 .
\end{aligned}
$$

Agora vamos mostrar a segunda parte da proposição. Note que se $D_{\gamma}=\{(\alpha, \beta)\}$, então $\alpha=\beta$ e 
$\gamma$ não pode ser a soma de duas raízes positivas que formam um ângulo obtuso entre si. Logo basta mostrar que se

(i) $\left|D_{\gamma}\right|=1$, ou

(ii) $\left|D_{\gamma}\right|=2$, e para todo o $(\alpha, \beta) \in D_{\gamma}, B(\alpha, \beta) \geq 0$, então $S_{\gamma}=0$.

Suponha que $D_{\gamma}=\{(\alpha, \beta)\}$. Segue que $\alpha=\beta$, logo temos que $m(\alpha, \beta)=\sqrt{2}$, e

$$
S_{\gamma}=\left(\sqrt{2} r_{\alpha}^{2}\right)^{2}-2 r_{\alpha}^{4}=0 .
$$

Agora suponha que a situação (ii) listada acima ocorre. Segue que $D_{\gamma}=\{(\alpha, \beta),(\beta, \alpha)\}$ com $\alpha \neq \beta$. Pela hipótese, temos que $m(\alpha, \beta)=m(\beta, \alpha)=1$, logo

$$
S_{\gamma}=\left(m(\alpha, \beta) r_{\alpha} r_{\beta}+m(\beta, \alpha) r_{\beta} r_{\alpha}\right)^{2}-2\left(r_{\alpha}^{2} r_{\beta}^{2}+r_{\beta}^{2} r_{\alpha}^{2}\right)=\left(2 r_{\alpha} r_{\beta}\right)^{2}-4 r_{\alpha}^{2} r_{\beta}^{2}=0 .
$$

Vamos estudar a soma dos $S_{\gamma}$ de maneira sistemática. Consideramos as quatro famílias clássicas de sistemas de raízes reduzidos separadamente, usando as realizações listadas no Apêndice A. Dividimos as raízes em conjuntos que são escritos de maneira similar e escrevemos os $r_{\alpha}$ para $\alpha$ em cada um desses conjuntos como coeficientes de matrizes (por exemplo, as raízes positivas de $D_{n}$ são divididas nos conjuntos $\left\{e_{i}-e_{j}\right\}$ e $\left\{e_{i}+e_{j}\right\}$, e tomamos matrizes triangulares superiores $A$ e $B$ com $r_{e_{i}-e_{j}}=A_{i j}$ e $r_{e_{i}+e_{j}}=B_{i j}$ ). Decompomos $2 R_{+}$nas somas desses conjuntos dois a dois. Em cada um desses subconjuntos, dividimos os elementos que atendem a condição da Proposição 2.2.6 em famílias que tem decomposições similares (por exemplo, dividimos os elementos de $\left\{e_{i}-e_{j}\right\}+\left\{e_{i}+e_{j}\right\}$ nas famílias $e_{i}+e_{j}+e_{k}-e_{l}$ com $i<j<k, l$, e $e_{i}+e_{j}$ com $i \leq j$ ). Finalmente, consideramos somatórios dos $S_{\gamma} \operatorname{com} \gamma$ variando dentro de cada uma dessas famílias. Para estimar cada um desses somatórios, somamos estimativas para os $S_{\gamma}$ em função dos coeficientes das matrizes construídas anteriormente, para então encontrar estimativas para os somatórios que dependem apenas da norma de Frobenius dessas matrizes (por exemplo, mostramos que $S_{e_{i}+e_{j}} \leq 8\left(A\left(B+B^{t}\right)+B A^{t}\right)_{i j}^{2}$ e que $\left.\sum_{i \leq j} S_{e_{i}+e_{j}} \leq 32\|A\|_{F}^{2}\|B\|_{F}^{2}\right)$.

A ordem em que os casos são apresentados foi escolhida para ir do mais simples (Caso $A_{n}$ ) ao mais complexo (Caso $B_{n}$ ). Muitos dos cálculos são similares, por isso algumas identidades e desigualdades só serão mostradas detalhadamente no Caso $A_{n}$. Como há várias semelhanças entre os casos, muitas referências a casos anteriores serão feitas durante a demonstração.

\section{Caso $A_{n}$}

Seja $A=\left(a_{i j}\right)$ a matriz $n+1 \times n+1$ com coeficientes

$$
a_{i j}= \begin{cases}r_{e_{i}-e_{j}} & \text { se } i<j, \\ 0 & \text { caso contrário, }\end{cases}
$$

Os $\gamma \in 2 R_{+}$podem ser divididos nas famílias

(1) $\gamma=e_{i}+e_{j}-e_{k}-e_{l} \operatorname{com} i<j<k<l$,

(2) $\gamma=e_{i}-e_{j} \operatorname{com} i<i+1<j$,

(3) $\gamma=e_{i}-e_{j}+e_{k}-e_{l} \operatorname{com} i<j<k<l$,

(4) $\gamma=2 e_{i}-e_{j}-e_{k} \operatorname{com} i<j \leq k$,

(5) $\gamma=e_{i}+e_{j}-2 e_{k} \operatorname{com} i<j<k$.

Pela Proposição 2.2.6 temos que $S_{\gamma}>0$ apenas se $\gamma$ for da família (1) ou (2). Portanto

$$
\sum_{\gamma \in 2 R_{+}} S_{\gamma}=\underbrace{\sum_{i<j<k<l} S_{e_{i}+e_{j}-e_{k}-e_{l}}}_{(\mathrm{A} .1)}+\underbrace{\sum_{i<i+1<j} S_{e_{i}-e_{j}}}_{(\mathrm{A} .2)} .
$$


Mostraremos que

$$
(\mathrm{A} .1) \leq 2\|A\|_{F}^{4} \quad \text { e } \quad(\mathrm{A} .2) \leq 8\|A\|_{F}^{4}
$$

onde $\|\cdot\|_{F}$ é a norma de Frobenius. Segue que

$$
\sum_{\gamma \in 2 R_{+}} S_{\gamma} \leq 10\|A\|_{F}^{4}
$$

Aplicando essas estimativas a (2.4) e usando o fato que $\|A\|_{F}^{2}=\sum_{i<j} r_{e_{i}-e_{j}}^{2}=\|f \cdot p\|^{2}=1$, temos

$$
\|\mathrm{II}(f \cdot p, f \cdot p)\|^{2} \leq 2+10\|A\|_{F}^{4}=12 .
$$

Estimando (A.1):

Primeiramente, note que se $\alpha, \beta \in R_{+}$são tais que $\alpha+\beta=e_{i}+e_{j}-e_{k}-e_{l}$ com $i<j<k<l$ então, a menos de permutações de $\alpha \operatorname{com} \beta$, devemos ter $\alpha=e_{i}-e_{k}$ e $\beta=e_{j}-e_{l}$ ou $\alpha=e_{i}-e_{l}$ e $\beta=e_{j}-e_{k}$. Como em ambos os casos $B(\alpha, \beta)=0$ temos que $m(\alpha, \beta)=1$ e portanto

$$
S_{e_{i}+e_{j}-e_{k}-e_{l}}=\left(2 a_{i k} a_{j l}+2 a_{i l} a_{j k}\right)^{2}-2\left(2 a_{i k}^{2} a_{j l}^{2}+2 a_{i l}^{2} a_{j k}^{2}\right)=8 a_{i k} a_{j l} a_{i l} a_{j k} .
$$

Como $a_{i k} a_{j l} a_{i l} a_{j k}$ é invariante por permutações do índice $i$ com o índice $j$, e por permutações do índice $k$ com o índice $l$, temos que

$$
\sum_{i<j<k<l} a_{i k} a_{j l} a_{i l} a_{j k}=\frac{1}{4} \sum_{\substack{i, j<k, l \\ i \neq j, k \neq l}} a_{i k} a_{j l} a_{i l} a_{j k}
$$

já que cada termo na soma à esquerda se repete quatro vezes na soma à direita. Usando o fato dos coeficientes de $A$ serem não negativos e escrevendo $\langle,\rangle_{F}$ para o produto de Frobenius, temos que

$$
\begin{aligned}
\sum_{i<j<k<l} a_{i k} a_{j l} a_{i l} a_{j k} & =\frac{1}{4} \sum_{\substack{i, j<k, l \\
i \neq j, k \neq l}} a_{i k} a_{j l} a_{i l} a_{j k} \\
& \leq \frac{1}{4} \sum_{i, j, k, l} a_{i k} a_{j l} a_{i l} a_{j k} \\
& =\frac{1}{4} \sum_{i, k} a_{i k} \sum_{j} a_{j k} \sum_{l} a_{j l} a_{i l} \\
& =\frac{1}{4} \sum_{i, k} a_{i k} \sum_{j} a_{j k}\left(A A^{t}\right)_{j i} \\
& =\frac{1}{4} \sum_{i, k} a_{i k}\left(A^{t} A A^{t}\right)_{k i} \\
& =\frac{1}{4}\left\langle A^{t}, A^{t} A A^{t}\right\rangle_{F} \\
& \leq \frac{1}{4}\|A\|_{F}\left\|A^{t} A A^{t}\right\|_{F} \\
& \leq \frac{1}{4}\|A\|_{F}^{4}
\end{aligned}
$$

(por Cauchy-Schwartz)

(já que $\|\cdot\|_{F}$ é multiplicativo).

Portanto

$$
\sum_{i<j<k<l} S_{e_{i}+e_{j}-e_{k}-e_{l}} \leq 8\left(\frac{1}{4}\|A\|_{F}^{4}\right)=2\|A\|_{F}^{4} .
$$

Estimando (A.2): 
Se $\alpha, \beta \in R_{+}$são tais que $\alpha+\beta=e_{i}-e_{j}$ com $i<j$ então, a menos de permutações de $\alpha$ com $\beta$, devemos ter $\alpha=e_{i}-e_{t}$ e $\beta=e_{t}-e_{j} \operatorname{com} i<t<j$. Como em todos os $\operatorname{casos} m(\alpha, \beta)=\sqrt{2}$ temos que

$$
S_{e_{i}-e_{j}}=\left(\sum_{t=i+1}^{j-1} 2 \sqrt{2} a_{i t} a_{t j}\right)^{2}-2 \sum_{t=i+1}^{j-1} 2 a_{i t}^{2} a_{t j}^{2} \leq 8\left(\sum_{t} a_{i t} a_{t j}\right)^{2}=8\left(A^{2}\right)_{i j}^{2} .
$$

Note que como $A$ é triangular estritamente superior (isso é, $A_{i j} \neq 0$ somente se $i<j$ ), temos que $\left(A^{2}\right)_{i j} \neq 0$ apenas se $i+1<j$. Então somando as estimativas para $S_{e_{i}-e_{j}}$ acima temos que

$$
(\mathrm{A} .2) \leq 8 \sum_{i<j}\left(A^{2}\right)_{i, j}^{2}=8 \sum_{i, j}\left(A^{2}\right)_{i, j}^{2}=8\left\|A^{2}\right\|_{F}^{2} \leq 8\|A\|_{F}^{4} .
$$

\section{Caso $D_{n}$}

Sejam $a_{i j}$ e $A$ como no Caso $A_{n-1}$. Seja $B=\left(b_{i j}\right)$ a matriz $n \times n$ com coeficientes

$$
b_{i j}= \begin{cases}r_{e_{i}+e_{j}} & \text { se } i<j, \\ 0 & \text { caso contrário. }\end{cases}
$$

Note que $\|A\|_{F}^{2}+\|B\|_{F}^{2}=\|f \cdot p\|^{2}=1$.

Escrevendo $\left\{e_{i}-e_{j}: 1 \leq i<j \leq n\right\}$ como $\left\{e_{i}-e_{j}\right\}$ e $\left\{e_{i}+e_{j}: 1 \leq i<j \leq n\right\}$ como $\left\{e_{i}+e_{j}\right\}$ temos que

$$
2 R_{+}=2\left\{e_{i}-e_{j}\right\} \cup\left(\left\{e_{i}-e_{j}\right\}+\left\{e_{i}+e_{j}\right\}\right) \cup 2\left\{e_{i}+e_{j}\right\}
$$

onde as uniões são disjuntas. Então temos que

$$
\sum_{\gamma \in 2 R_{+}} S_{\gamma}=\sum_{\gamma \in 2\left\{e_{i}-e_{j}\right\}} S_{\gamma}+\underbrace{\sum_{\gamma \in\left\{e_{i}-e_{j}\right\}+\left\{e_{i}+e_{j}\right\}} S_{\gamma}}_{\text {(D.I) }}+\underbrace{\sum_{\gamma \in 2\left\{e_{i}+e_{j}\right\}} S_{\gamma}}_{\text {(D.II) }} .
$$

Segue da decomposição de $2 R_{+}$acima que se $\alpha, \beta \in R_{+}$são tais que $\alpha+\beta \in 2\left\{e_{i}-e_{j}\right\}$ então $\alpha, \beta \in\left\{e_{i}-e_{j}\right\}$. Logo, de maneira análoga ao Caso $A_{n}$, temos que

$$
\sum_{\gamma \in 2\left\{e_{i}-e_{j}\right\}} S_{\gamma} \leq 10\|A\|_{F}^{4}
$$

A seguir vamos mostrar que

$$
\text { (D.I) } \leq 56\|A\|_{F}^{2}\|B\|_{F}^{2} \quad \text { e } \quad(\text { D.II }) \leq 18\|B\|_{F}^{4} .
$$

Escrevendo $X=\|A\|_{F}^{2}$ e $Y=\|B\|_{F}^{2}$ temos que

$$
\begin{aligned}
\sum_{\gamma \in 2 R_{+}} S_{\gamma} & \leq 10 X^{2}+56 X Y+18 Y^{2} \\
& \leq 10(X+Y)^{2}+36 X Y+8 Y^{2} \\
& =10+4\left(9 X Y+2 Y^{2}\right) .
\end{aligned}
$$

Como $X+Y=1$ temos que $9 X Y+2 Y^{2}=9 Y-7 Y^{2} \leq 3$, logo $\sum_{\gamma \in 2 R_{+}} S_{\gamma} \leq 22$. Aplicando essa estimativa a (2.4) temos que

$$
\|\mathrm{II}(f \cdot p, f \cdot p)\|^{2} \leq 24
$$


Estimando (D.I):

Suponha $\gamma \in\left\{e_{i}-e_{j}\right\}+\left\{e_{i}+e_{j}\right\}$ é tal que existem mais de dois $(\alpha, \beta) \in R_{+} \times R_{+}$tais que $\alpha+\beta=\gamma$ ou que $\gamma$ é a soma de raízes positivas que formam um ângulo obtuso entre si. Então

(1) $\gamma=e_{i}+e_{j}-e_{l}+e_{k} \operatorname{com} i<j<l<k$, ou

(2) $\gamma=e_{i}+e_{j}+e_{k}-e_{l}$ com $i<j<k<l$, ou

(3) $\gamma=e_{i}+e_{j} \operatorname{com} i \leq j$.

Logo

$$
(\mathrm{D} . \mathrm{I})=\underbrace{\sum_{i<j<l<k} S_{e_{i}+e_{j}-e_{l}+e_{k}}}_{\text {(D.I.1) }}+\underbrace{\sum_{i<j<k<l} S_{e_{i}+e_{j}+e_{k}-e_{l}}}_{\text {(D.I.2) }}+\underbrace{\sum_{i \leq j} S_{e_{i}+e_{j}}}_{\text {(D.I.3) }} .
$$

Mostraremos que

$$
(\text { D.I.1 })+(\text { D.I. } 2) \leq 24\|A\|_{F}^{2}\|B\|_{F}^{2} \quad \text { e } \quad(\text { D.I.3 }) \leq 32\|A\|_{F}^{2}\|B\|_{F}^{2} .
$$

Estimando (D.I.1) + (D.I.2):

Note que se $\alpha+\beta=e_{i}+e_{j}-e_{l}+e_{k}$ com $i<j<l<k$ então, a menos de permutações, devemos ter $\alpha=e_{i}-e_{l}$ e $\beta=e_{j}+e_{k}$ ou $\alpha=e_{i}+e_{k}$ e $\beta=e_{j}-e_{l}$. Como em todos esses casos $m(\alpha, \beta)=1$, temos que

$$
S_{e_{i}+e_{j}-e_{l}+e_{k}}=8 a_{i l} b_{j k} b_{i k} a_{j l} .
$$

Se $\alpha+\beta=e_{i}+e_{j}+e_{k}-e_{l}$ com $i<j<k<l$ então, a menos de permutações, devemos ter $\alpha=e_{i}+e_{j}$ e $\beta=e_{k}-e_{l}$ ou $\alpha=e_{i}+e_{k}$ e $\beta=e_{j}-e_{l}$ ou $\alpha=e_{i}-e_{l}$ e $\beta=e_{j}+e_{k}$. Como em todos esses casos $m(\alpha, \beta)=1$, temos que

$$
S_{e_{i}+e_{j}+e_{k}-e_{l}}=8\left(b_{i j} a_{k l} b_{i k} a_{j l}+b_{i j} a_{k l} a_{i l} b_{j k}+b_{i k} a_{j l} a_{i l} b_{j k}\right) .
$$

Note que

$$
\begin{aligned}
(\text { D.I.1 })+(\text { D.I.2 }) & =8 \sum_{i<j<l<k} a_{i l} b_{j k} b_{i k} a_{j l}+8 \sum_{i<j<k<l} b_{i j} a_{k l} b_{i k} a_{j l}+b_{i j} a_{k l} a_{i l} b_{j k}+b_{i k} a_{j l} a_{i l} b_{j k} \\
& =8\left(\sum_{i<j<k<l} b_{i j} a_{k l} b_{i k} a_{j l}+\sum_{i<j<k<l} b_{i j} a_{k l} a_{i l} b_{j k}+\sum_{\substack{i<j<k, l \\
k \neq l}} b_{i k} a_{j l} a_{i l} b_{j k}\right) .
\end{aligned}
$$

Agora note que

$$
\sum_{i<j<k<l} b_{i j} a_{k l} b_{i k} a_{j l} \leq \sum_{i, j, k, l} b_{i j} a_{k l} b_{i k} a_{j l}=\left\langle B, B A A^{t}\right\rangle_{F} \leq\|A\|_{F}^{2}\|B\|_{F}^{2} .
$$

Similarmente

$$
\sum_{i<j<k<l} b_{i j} a_{k l} a_{i l} b_{j k} \leq \sum_{i, j, k, l} b_{i j} a_{k l} a_{i l} b_{j k}=\left\langle B^{t}, B A A^{t}\right\rangle_{F} \leq\|A\|_{F}^{2}\|B\|_{F}^{2}
$$

e

$$
\sum_{\substack{i<j<k, l \\ k \neq l}} b_{i k} a_{j l} a_{i l} b_{j k} \leq \sum_{i, j, k, l} b_{i k} a_{j l} a_{i l} b_{j k}=\left\langle B^{t}, B^{t} A A^{t}\right\rangle_{F} \leq\|A\|_{F}^{2}\|B\|_{F}^{2}
$$

Segue que

$$
\text { (D.I.1) }+\left(\text { D.I.2) } \leq 24\|A\|_{F}^{2}\|B\|_{F}^{2}\right. \text {. }
$$


Estimando (D.I.3):

Se $\alpha+\beta=2 e_{i}$ então, a menos de permutações, devemos ter $\alpha=e_{i}-e_{t}$ e $\beta=e_{i}+e_{t}$ com $i<t \leq n$. Como em todos esses casos $m(\alpha, \beta)=1$, temos que

$$
S_{2 e_{i}} \leq\left(2 \sum_{t} a_{i t} b_{i t}\right)^{2}=4\left(A B^{t}\right)_{i i}^{2} .
$$

Se $\alpha+\beta=e_{i}+e_{j} \operatorname{com} i<j$ então, a menos de permutações, devemos ter $\alpha=e_{i}-e_{t}$ e $\beta=e_{j}+e_{t}$ $\operatorname{com} i<t \leq n, t \neq j$ ou então $\alpha=e_{i}+e_{t}$ e $\beta=e_{j}-e_{t} \operatorname{com} j<t \leq n$. Note que em todos esses casos $m(\alpha, \beta)=\sqrt{2}$. Lembrando que $r_{e_{j}+e_{t}}=b_{j t}+b_{t j}$, temos

$$
S_{e_{i}+e_{j}} \leq 8\left(\sum_{t} a_{i t}\left(b_{t j}+b_{j t}\right)+\sum_{t} b_{i t} a_{j t}\right)^{2}=8\left(A\left(B+B^{t}\right)+B A^{t}\right)_{i j}^{2} .
$$

Como os coeficientes de $A$ e de $B$ são não negativos temos que

$$
\left(A B^{t}\right)_{i i}^{2} \leq\left(A\left(B+B^{t}\right)+\left(B+B^{t}\right) A^{t}\right)_{i i}^{2} \quad \text { e } \quad\left(A\left(B+B^{t}\right)+B A^{t}\right)_{i j}^{2} \leq\left(A\left(B+B^{t}\right)+\left(B+B^{t}\right) A^{t}\right)_{i j}^{2} .
$$

Portanto

$$
(\text { D.I.3 }) \leq 4 \sum_{i}\left(A\left(B+B^{t}\right)+\left(B+B^{t}\right) A^{t}\right)_{i i}^{2}+8 \sum_{i<j}\left(A\left(B+B^{t}\right)+\left(B+B^{t}\right) A^{t}\right)_{i j}^{2} .
$$

Como $A\left(B+B^{t}\right)+\left(B+B^{t}\right) A^{t}$ é simétrica temos que

$$
8 \sum_{i<j}\left(A\left(B+B^{t}\right)+\left(B+B^{t}\right) A^{t}\right)_{i j}^{2}=4 \sum_{i \neq j}\left(A\left(B+B^{t}\right)+\left(B+B^{t}\right) A^{t}\right)_{i j}^{2}
$$

e portanto

$$
\left.(\text { D.I.3 }) \leq 4 \sum_{i, j}\left(A\left(B+B^{t}\right)+\left(B+B^{t}\right) A^{t}\right)_{i j}^{2}=4 \| A\left(B+B^{t}\right)+\left(B+B^{t}\right) A^{t}\right) \|_{F}^{2} .
$$

Agora note que

$$
4\left\|A\left(B+B^{t}\right)+\left(B+B^{t}\right) A^{t}\right\|_{F}^{2} \leq 4\left(2\left\|A\left(B+B^{t}\right)\right\|_{F}\right)^{2} \leq 16\|A\|_{F}^{2}\left\|\left(B+B^{t}\right)\right\|_{F}^{2} .
$$

Como $B$ é triangular estritamente superior temos que $\left\|B+B^{t}\right\|_{F}^{2}=2\|B\|_{F}^{2}$ e portanto

$$
\sum_{i \leq j} S_{e_{i}+e_{j}} \leq 32\|A\|_{F}^{2}\|B\|_{F}^{2}
$$

Estimando (D.II):

Primeiramente note que se $\alpha+\beta \in 2\left\{e_{i}+e_{j}\right\}$, então $\alpha, \beta \in\left\{e_{i}+e_{j}\right\}$, e dois elementos de $\left\{e_{i}+e_{j}\right\}$ não podem formar um ângulo obtuso. Assuma que $\gamma \in 2\left\{e_{i}+e_{j}\right\}$ é tal que existem mais de dois $(\alpha, \beta) \in R_{+}$tais que $\alpha+\beta=\gamma$. Segue que $\gamma=e_{i}+e_{j}+e_{k}+e_{l}$ com $i<j<k<l$ e portanto

$$
\sum_{\gamma \in 2\left\{e_{i}+e_{j}\right\}} S_{\gamma}=\sum_{i<j<k<l} S_{e_{i}+e_{j}+e_{k}+e_{l}} .
$$

Se $\alpha, \beta \in R_{+}$são tais que $\alpha+\beta=e_{i}+e_{j}+e_{k}+e_{l}$ então, a menos de permutações, devemos ter $\alpha=e_{i}+e_{j}$ e $\beta=e_{k}+e_{l}$ ou $\alpha=e_{i}+e_{k}$ e $\beta=e_{j}+e_{l}$ ou $\alpha=e_{i}+e_{l}$ e $\beta=e_{j}+e_{k}$. Como em todos os casos $m(\alpha, \beta)=1$ temos que

$$
S_{e_{i}+e_{j}+e_{k}+e_{l}}=8\left(b_{i j} b_{k l} b_{i k} b_{j l}+b_{i j} b_{k l} b_{i l} b_{j k}+b_{i k} b_{j l} b_{i l} b_{j k}\right) .
$$


Note que $b_{i k} b_{j l} b_{i l} b_{j k}$ é invariante se permutarmos $i \operatorname{com} j$ ou $k$ com $l$. Então de maneira similar a como foi feito na estimativa de (A.1), temos que

$$
\sum_{i<j<k<l} b_{i k} b_{j l} b_{i l} b_{j k} \leq \frac{1}{4} \sum_{i, j, k, l} b_{i k} b_{j l} b_{i l} b_{j k} \leq \frac{1}{4}\left\langle B^{t}, B^{t} B B^{t}\right\rangle_{F} \leq \frac{1}{4}\|B\|_{F}^{4} .
$$

Adicionalmente

$$
\sum_{i<j<k<l} b_{i j} b_{k l} b_{i k} b_{j l} \leq \sum_{i, j, k, l} b_{i j} b_{k l} b_{i k} b_{j l}=\left\langle B^{2}, B^{2}\right\rangle_{F} \leq\|B\|_{F}^{4}
$$

e

$$
\sum_{i<j<k<l} b_{i j} b_{k l} b_{i l} b_{j k} \leq \sum_{i, j, k, l} b_{i j} b_{k l} b_{i l} b_{j k}=\left\langle B^{2}, B B^{t}\right\rangle_{F} \leq\|B\|_{F}^{4} .
$$

Somando essas estimativas temos que

$$
\sum_{\gamma \in 2\left\{e_{i}+e_{j}\right\}} S_{\gamma} \leq 8\left(\|B\|_{F}^{4}+\|B\|_{F}^{4}+\frac{1}{4}\|B\|_{F}^{4}\right)=18\|B\|_{F}^{4} .
$$

\section{Caso $C_{n}$}

Sejam $a_{i j}, A, b_{i j}$ e $B$ como no Caso $D_{n}$, exceto que

$$
b_{i i}=r_{2 e_{i}} .
$$

Tomando $\left\{e_{i}+e_{j}\right\}=\left\{e_{i}+e_{j}: 1 \leq i \leq j \leq n\right\}$ temos, analogamente ao Caso $D_{n}$, que

$$
2 R_{+}=2\left\{e_{i}-e_{j}\right\} \cup\left(\left\{e_{i}-e_{j}\right\}+\left\{e_{i}+e_{j}\right\}\right) \cup 2\left\{e_{i}+e_{j}\right\},
$$

onde as uniões são disjuntas. Então temos que

$$
\sum_{\gamma \in 2 R_{+}} S_{\gamma}=\sum_{\gamma \in 2\left\{e_{i}-e_{j}\right\}} S_{\gamma}+\underbrace{\sum_{\gamma \in\left\{e_{i}-e_{j}\right\} \cup\left\{e_{i}+e_{j}\right\}} S_{\gamma}}_{\text {(C.I) }}+\underbrace{\sum_{\gamma \in 2\left\{e_{i}+e_{j}\right\}} S_{\gamma}}_{(\text {C.II })} .
$$

Pela decomposição de $2 R_{+}$apresentada acima, temos que se $\alpha+\beta \in 2\left\{e_{i}-e_{j}\right\}$ então $\alpha, \beta \in$ $\left\{e_{i}-e_{j}\right\}$. Logo, de maneira análoga ao Caso $A_{n}$ temos que

$$
\sum_{\gamma \in 2\left\{e_{i}-e_{j}\right\}} S_{\gamma} \leq 10\|A\|_{F}^{4}
$$

Mostraremos a seguir que, de maneira análoga ao Caso $D_{n}$, temos que

$$
(\mathrm{C} . \mathrm{I}) \leq 56\|A\|_{F}^{2}\|B\|_{F}^{2} \quad \text { e } \quad(\mathrm{C} . \mathrm{II}) \leq 18\|B\|_{F}^{4} .
$$

Portanto

$$
\|\mathrm{II}(f \cdot p, f \cdot p)\|^{2} \leq 24
$$

Estimando (C.I):

Baseamo-nos na estimativa de (D.I). Suponha que $\gamma \in\left\{e_{i}-e_{j}\right\} \cup\left\{e_{i}+e_{j}\right\}$ é tal que existem mais do que dois $(\alpha, \beta) \in R_{+} \times R_{+}$tais que $\alpha+\beta=\gamma$ ou então que $\gamma$ é a soma de raízes positivas que formam um ângulo obtuso entre si. Então devemos ter

(1) $\gamma=e_{i}+e_{j}-e_{l}+e_{k} \operatorname{com} i<j<l<k$, ou

(2) $\gamma=e_{i}+e_{j}+e_{k}-e_{l}$ com $i<j<k<l$, ou 
(3) $\gamma=e_{i}+2 e_{j}-e_{l}$ com $i<j<l$, ou

(4) $\gamma=2 e_{i}+e_{k}-e_{l}$ com $i<k<l$, ou

(5) $\gamma=e_{i}+e_{j} \operatorname{com} i \leq j$.

Então (C.I) é igual a

$$
\underbrace{\sum_{i<j<l<k} S_{e_{i}+e_{j}-e_{l}+e_{k}}}_{\text {(C.I.1) }}+\underbrace{\sum_{i<j<k<l} S_{e_{i}+e_{j}+e_{k}-e_{l}}}_{\text {(C.I.2) }}+\underbrace{\sum_{i<j<l} S_{e_{i}+2 e_{j}-e_{l}}}_{\text {(C.I.3) }}+\underbrace{\sum_{i<k<l} S_{2 e_{i}+e_{k}-e_{l}}}_{\text {(C.I.4) }}+\underbrace{\sum_{i \leq j} S_{e_{i}+e_{j}}}_{\text {(C.I.5) }} .
$$

Mostraremos que

$$
(\text { C.I.1 })+(\text { C.I.2 })+(\text { C.I.3 })+(\text { C.I.4 }) \leq 24\|A\|_{F}^{2}\|B\|_{F}^{2} \quad \text { e } \quad(\text { C.I.5 }) \leq 32\|A\|_{F}^{2}\|B\|_{F}^{2} .
$$

Estimando (C.I.1) + (C.I.2) + (C.I.3) + (C.I.4):

Repetindo os argumentos usados na estimativa de (D.I.1) + (D.I.2), temos que

$$
(\text { C.I.1 })+(\text { C.I.2) } \leq 8 \sum_{i<j<k<l} b_{i j} a_{k l} b_{i k} a_{j l}+\underbrace{8 \sum_{i<j<k<l} b_{i j} a_{k l} a_{i l} b_{j k}}_{(*)}+8 \sum_{\substack{i<j<k, l \\ k \neq l}} b_{i k} a_{j l} a_{i l} b_{j k} .
$$

Para obter a estimativa desejada adicionaremos (C.I.3) e (C.I.4) a (*). Se $\alpha+\beta=e_{i}+2 e_{j}-e_{l}$ com $i<j<l$ então, a menos de permutações, devemos ter $\alpha=e_{i}+e_{j}$ e $\beta=e_{j}-e_{l}$ ou $\alpha=e_{i}-e_{l}$ e $\beta=2 e_{j}$. Como em ambos os casos $m(\alpha, \beta)=1$, temos que

$$
S_{e_{i}+2 e_{j}-e_{l}}=8 b_{i j} a_{j l} a_{i l} b_{j j} .
$$

Similarmente

$$
S_{2 e_{i}+e_{k}-e_{l}}=8 b_{i i} a_{k l} b_{i k} a_{i l} \quad \text { se } i<k<l .
$$

Note que se $j=k$ então $b_{i j} a_{k l} a_{i l} b_{j k}=b_{i j} a_{j l} a_{i l} b_{j j}$ e se $i=j$ então $b_{i j} a_{k l} b_{i k} a_{j l}=b_{i i} a_{k l} b_{i k} a_{i l}$. Portanto temos que $(*)+($ C.I.3) + (C.I.4) é igual a

$$
8 \sum_{i<j<k<l} b_{i j} a_{k l} a_{i l} b_{j k}+8 \sum_{i<j=k<l} b_{i j} a_{k l} a_{i l} b_{j k}+8 \sum_{i=j<k<l} b_{i j} a_{k l} b_{i k} a_{j l} \leq 8 \sum_{i, j, k, l} b_{i j} a_{k l} a_{i l} b_{j k} .
$$

Então

$$
(\text { C.I.1 })+(\text { C.I.2 })+(\text { C.I.3 })+(\text { C.I. } 4) \leq 8 \sum_{i, j, k, l}\left(b_{i j} a_{k l} b_{i k} a_{j l}+b_{i j} a_{k l} a_{i l} b_{j k}+b_{i k} a_{j l} a_{i l} b_{j k}\right) .
$$

Repetindo os argumentos finais da estimativa de (D.I.1) + (D.I.2) temos que

$$
(\text { C.I.1 })+(\text { C.I.2 })+(\text { C.I.3 })+(\text { C.I.4 }) \leq 24\|A\|_{F}^{2}\|B\|_{F}^{2} .
$$

\section{Estimando (C.I.5):}

Repetindo os argumentos usados na estimativa de (D.I.3) temos que

$$
S_{2 e_{i}} \leq\left(2 \sum_{t} a_{i, t} b_{i, t}\right)^{2}=4\left(A B^{t}\right)_{i i}^{2}
$$

Se $\alpha, \beta$ são tais que $\alpha+\beta=e_{i}+e_{j} \operatorname{com} i<j$ então, a menos de permutações, $\alpha$ e $\beta$ são as 
raízes listadas na estimativa de (D.I.3) ou então $\alpha=e_{i}-e_{j}$ e $\beta=2 e_{j}$. Segue que

$$
S_{e_{i}+e_{j}} \leq 8\left(\sum_{t ; t \neq j} a_{i t}\left(b_{t j}+b_{j t}\right)+a_{i j} b_{j j}+\sum_{t} b_{i t} a_{j t}\right)^{2}
$$

Seja $\tilde{B}$ a matriz $n \times n$ com coeficientes $\tilde{B}_{t j}=b_{t j}+b_{j t}$ se $t \neq j$ e $\tilde{B}_{j j}=b_{j j}$. Então temos que $S_{e_{i}+e_{j}} \leq 8\left(A \tilde{B}+B A^{t}\right)_{i j}^{2}$. Por argumentos análogos aos usados na estimativa de (D.I.3), temos que

$$
\sum_{i \leq j} S_{e_{i}+e_{j}} \leq 16\|A\|_{F}^{2}\|\tilde{B}\|_{F}^{2}
$$

Como

$$
\|\tilde{B}\|_{F}^{2}=2 \sum_{i \neq j} b_{i j}^{2}+\sum_{i} b_{i i}^{2} \leq 2 \sum_{i, j} b_{i j}^{2}=2\|B\|_{F}^{2}
$$

segue que

$$
\sum_{i \leq j} S_{e_{i}+e_{j}} \leq 32\|A\|_{F}^{2}\|B\|_{F}^{2}
$$

Estimando (C.II):

Note que $\alpha+\beta \in 2\left\{e_{i}+e_{j}\right\}$ apenas se $\alpha, \beta \in\left\{e_{i}+e_{j}\right\}$ e que que dois elementos de $\left\{e_{i}+e_{j}\right\}$ não podem formar um ângulo obtuso. Se $\gamma \in 2\left\{e_{k}+e_{l}\right\}$ é tal que existem mais de dois $(\alpha, \beta) \in R_{+} \times R_{+}$ tais que $\alpha+\beta=\gamma$, então

(1) $\gamma=e_{i}+e_{j}+e_{k}+e_{l}$ para $i<j<k<l$, ou

(2) $\gamma=2 e_{i}+e_{k}+e_{l}$ para $i<k<l$, ou

(3) $\gamma=e_{i}+2 e_{j}+e_{l}$ para $i<j<l$, ou

(4) $\gamma=e_{i}+e_{j}+2 e_{k}$ para $i<j<k$, ou

(5) $\gamma=2 e_{i}+2 e_{k}$ para $i<k$.

Logo

$$
(\mathrm{C.II})=\underbrace{\sum_{i<j<k<l} S_{e_{i}+e_{j}+e_{k}+e_{l}}}_{(\text {C.II.1) }}+\underbrace{\sum_{i<k<l} S_{2 e_{i}+e_{k}+e_{l}}}_{(\text {C.II.2) }}+\underbrace{\sum_{i<j<l} S_{e_{i}+2 e_{j}+e_{l}}}_{\text {(C.II.3) }}+\underbrace{\sum_{i<j<k} S_{e_{i}+e_{j}+2 e_{k}}}_{(\text {C.II.4) }}+\underbrace{\sum_{i<k} S_{2 e_{i}+2 e_{k}}}_{(\text {C.II.5) }} .
$$

Repetindo os argumentos da estimativa de (D.II) temos que

$$
S_{e_{i}+e_{j}+e_{k}+e_{l}}=8\left(b_{i j} b_{k l} b_{i k} b_{j l}+b_{i j} b_{k l} b_{i l} b_{j k}+b_{i k} b_{j l} b_{i l} b_{j k}\right) \quad \text { se } i<j<k<l .
$$

Então

$$
(\text { C.II.1 })=8 \sum_{i<j<k<l} b_{i j} b_{k l} b_{i k} b_{j l}+\underbrace{8 \sum_{i<j<k<l} b_{i j} b_{k l} b_{i l} b_{j k}}_{(*)}+8 \sum_{i<j<k<l} b_{i k} b_{j l} b_{i l} b_{j k} .
$$

Para obter a estimativa desejada adicionaremos (C.II.2), (C.II.3), (C.II.4) e (C.II.5) a (*). Se $\alpha, \beta \in R_{+}$são tais que $\alpha+\beta=2 e_{i}+e_{k}+e_{l} \operatorname{com} i<k<l$ então, a menos de permutações, devemos ter $\alpha=2 e_{i}$ e $\beta=e_{k}+e_{l}$ ou $\alpha=e_{i}+e_{k}$ e $\beta=e_{i}+e_{l}$. Como em ambos esses casos $m(\alpha, \beta)=1$, temos que

$$
S_{2 e_{i}+e_{k}+e_{l}}=8 b_{i i} b_{k l} b_{i k} b_{i l}
$$

Similarmente temos que

$$
S_{e_{i}+2 e_{j}+e_{l}}=8 b_{i j} b_{j l} b_{i l} b_{j j} \text { se } i<j<l \quad \text { e } \quad S_{e_{i}+e_{j}+2 e_{k}}=8 b_{i j} b_{k k} b_{i k} b_{j k} \text { se } i<j<k \text {. }
$$


Se $\alpha, \beta \in R_{+}$são tais que $\alpha+\beta=2 e_{i}+2 e_{k}$ com $i<k$ então, a menos de permutações, devemos ter $\alpha=2 e_{i}$ e $\beta=2 e_{k}$ ou $\alpha=\beta=e_{i}+e_{k}$. Como $m\left(2 e_{i}, 2 e_{k}\right)=1$ e $m\left(e_{i}+e_{k}, e_{i}+e_{k}\right)=\sqrt{2}$, temos que

$$
S_{2 e_{i}+2 e_{k}}=4 \sqrt{2} b_{i i} b_{k k} b_{i k} b_{i k}
$$

Então

$$
8 b_{i j} b_{k l} b_{i l} b_{j k}= \begin{cases}S_{2 e_{i}+e_{k}+e_{l}} & \text { se } i=j<k<l, \\ S_{e_{i}+2 e_{j}+e_{l}} & \text { se } i<j=k<l, \\ S_{e_{i}+e_{j}+2 e_{k}} & \text { se } i<j<k=l, \\ \sqrt{2} S_{2 e_{i}+2 e_{k}} & \text { se } i=j<k=l .\end{cases}
$$

$\mathrm{e}(*)+($ C.II. 2$)+($ C.II.3) $+($ C.II.4) $+($ C.II.5) é igual a

$$
\begin{aligned}
8 \sum_{i<j<k<l} b_{i j} b_{k l} b_{i l} b_{j k}+8 & \sum_{i=j<k<l} b_{i j} b_{k l} b_{i l} b_{j k}+8 \sum_{i<j=k<l} b_{i j} b_{k l} b_{i l} b_{j k} \\
& +8 \sum_{i<j<k=l} b_{i j} b_{k l} b_{i l} b_{j k}+4 \sqrt{2} \sum_{i=j<k=l} b_{i j} b_{k l} b_{i l} b_{j k} \leq 8 \sum_{i, j, k, l} b_{i j} b_{k l} b_{i l} b_{j k} .
\end{aligned}
$$

Segue que

$$
(\mathrm{C} . \mathrm{II}) \leq 8 \sum_{i<j<k<l} b_{i j} b_{k l} b_{i k} b_{j l}+8 \sum_{i, j, k, l} b_{i j} b_{k l} b_{i l} b_{j k}+8 \sum_{i<j<k<l} b_{i k} b_{j l} b_{i l} b_{j k}
$$

Repetindo os argumentos finais da estimativa de (D.II), temos que (C.II) $\leq 18\|B\|_{F}^{4}$.

\section{Caso $B_{n}$}

Sejam $a_{i j}, A, b_{i j}, B$ como no Caso $D_{n}$. Seja $c_{i}=r_{e_{i}}$ e $C$ o vetor coluna que tem os $c_{i}$ como coeficientes. Note que $\|A\|_{F}^{2}+\|B\|_{F}^{2}+\|C\|_{F}^{2}=1$. Temos

$$
2 R_{+}=2\left\{e_{i}-e_{j}\right\} \cup\left(\left\{e_{i}-e_{j}\right\}+\left\{e_{i}+e_{j}\right\}\right) \cup 2\left\{e_{i}+e_{j}\right\} \cup\left(\left\{e_{i}\right\}+\left\{e_{i}-e_{j}\right\}\right) \cup\left(\left\{e_{i}\right\}+\left\{e_{i}+e_{j}\right\}\right)
$$

onde todas as uniões são disjuntas (note que $2\left\{e_{i}\right\} \subset\left\{e_{i}-e_{j}\right\}+\left\{e_{i}+e_{j}\right\}$ ). Então

$$
\sum_{\gamma \in 2 R_{+}} S_{\gamma}=\sum_{\gamma \in 2\left\{e_{i}-e_{j}\right\} \cup 2\left\{e_{i}+e_{j}\right\}} S_{\gamma}+\underbrace{\sum_{\gamma \in\left\{e_{i}-e_{j}\right\}+\left\{e_{i}+e_{j}\right\}} S_{\gamma}}_{\text {(B.I) }}+\underbrace{\sum_{\gamma \in\left\{e_{i}\right\}+\left\{e_{i}-e_{j}\right\}} S_{\gamma}}_{\text {(B.II) }}+\underbrace{\sum_{\gamma \in\left\{e_{i}\right\}+\left\{e_{i}+e_{j}\right\}} S_{\gamma}}_{\text {(B.II) }} .
$$

Segue da decomposição de $2 R_{+}$apresentada acima que se $\alpha, \beta \in R_{+}$são tais que $\alpha+\beta \in$ $2\left\{e_{i}-e_{j}\right\} \cup 2\left\{e_{i}+e_{j}\right\}$, então $\alpha, \beta \in\left\{e_{i}-e_{j}\right\}$ ou $\alpha, \beta \in\left\{e_{i}+e_{j}\right\}$. Logo, de maneira análoga ao Caso $D_{n}$, temos que:

$$
\sum_{\gamma \in 2\left\{e_{i}-e_{j}\right\} \cup 2\left\{e_{i}+e_{j}\right\}} S_{\gamma} \leq 10\|A\|_{F}^{4}+18\|B\|_{F}^{4} .
$$

Mostraremos que

$$
\begin{aligned}
& (\mathrm{B} . \mathrm{I}) \leq 56\|A\|_{F}^{2}\|B\|_{F}^{2}+16\|A\|_{F}\|B\|_{F}\|C\|_{F}^{2}+2\|C\|_{F}^{4}, \\
& (\mathrm{~B} . \mathrm{II}) \leq 12\|A\|_{F}^{2}\|C\|_{F}^{2} \quad \text { e } \quad(\mathrm{B} . \mathrm{III}) \leq 16\|B\|_{F}^{2}\|C\|_{F}^{2} .
\end{aligned}
$$

Escrevendo $X=\|A\|_{F}^{2}, Y=\|B\|_{F}^{2}, Z=\|C\|_{F}^{2}$ e tomando essas estimativas temos que

$$
\sum_{\gamma \in 2 R_{+}} S_{\gamma} \leq \underbrace{10 X^{2}+18 Y^{2}+56 X Y}_{p(X, Y)}+2 Z^{2}+Z(\underbrace{16 \sqrt{X Y}+12 X+16 Y}_{q(X, Y)}) .
$$


Do Caso $D_{n}$ sabemos que com as restrições $X+Y=1$ e $X, Y>0$ temos que $p(X, Y) \leq 22$. Então, como $p$ é homogêneo de grau 2, se tomarmos as restrições $X+Y=1-Z$ e $X, Y>0$, temos que $p(X, Y) \leq 22(1-Z)^{2}$. Agora note que $q(X, Y) \leq 16(\sqrt{X Y}+X+Y)$. Como $X+Y=1-Z$ temos que $\sqrt{X Y}+X+Y \leq \frac{3}{2}(1-Z), \log q q(X, Y) \leq 24(1-Z)$. Usando essas estimativas temos que

$$
\sum_{\gamma \in 2 R_{+}} S_{\gamma} \leq 22(1-Z)^{2}+2 Z^{2}+24 Z(1-Z)=22-20 Z \leq 22 .
$$

Então segue de (2.4) que $\|\mathrm{II}(f . p, f . p)\|^{2} \leq 24$.

Estimando (B.I):

De maneira análoga ao caso Caso $D_{n}$, temos que se $\gamma \in\left\{e_{i}-e_{j}\right\}+\left\{e_{i}+e_{j}\right\}$ é tal que existem mais de dois $(\alpha, \beta) \in R_{+} \times R_{+}$tais que $\alpha+\beta=\gamma$ ou se $\gamma$ é a soma de raízes positivas que formam um ângulo obtuso entre si, então

(1) $\gamma=e_{i}+e_{j}-e_{l}+e_{k} \operatorname{com} i<j<l<k$, ou

(2) $\gamma=e_{i}+e_{j}+e_{k}-e_{l}$ com $i<j<k<l$, ou

(3) $\gamma=e_{i}+e_{j} \operatorname{com} i \leq j$.

Segue que

$$
(\mathrm{B.I})=\underbrace{\sum_{i<j<l<k} S_{e_{i}+e_{j}-e_{l}+e_{k}}}_{\text {(B.I.1) }}+\underbrace{\sum_{i<j<k<l} S_{e_{i}+e_{j}+e_{k}-e_{l}}}_{\text {(B.I.2) }}+\underbrace{\sum_{i \leq j} S_{e_{i}+e_{j}}}_{(\text {B.I.3) }} .
$$

Note que as decomposições em somas de raízes positivas dos $\gamma=e_{i}+e_{j}-e_{l}+e_{k} \operatorname{com} i<j<l<k$ e $\operatorname{dos} \gamma=e_{i}+e_{j}+e_{k}-e_{l} \operatorname{com} i<j<k<l$ são as mesmas que no Caso $D_{n}$. Então, repetindo os argumentos usadas na estimativa de (D.I.1) + (D.I.2), temos que

$$
\left(\text { B.I.1) }+\left(\text { B.I.2) } \leq 24\|A\|_{F}^{2}\|B\|_{F}^{2} .\right.\right.
$$

Agora note que se $\gamma=e_{i}+e_{j} \operatorname{com} i \leq j$ então os $\alpha, \beta \in R_{+}$tais que $\alpha+\beta=\gamma$ são, a menos permutações, os $\alpha$ e $\beta$ listados na estimativa de (D.I.3) e mais $\alpha=e_{i}$ e $\beta=e_{j}$. Então, como $m\left(e_{i}, e_{i}\right)=\sqrt{2}$ temos que

$$
S_{2 e_{i}} \leq\left(2 \sum_{t} a_{i t} b_{i t}+\sqrt{2} c_{i}^{2}\right)^{2}=2\left(\sqrt{2} A B^{t}+C C^{t}\right)_{i i}^{2} .
$$

Como $m\left(e_{i}, e_{j}\right)=1$ para $i<j$, segue que

$$
S_{e_{i}+e_{j}} \leq\left(2 \sqrt{2} \sum_{t} a_{i t}\left(b_{t j}+b_{j t}\right)+2 \sqrt{2} \sum_{t} b_{i t} a_{j t}+2 c_{i} c_{j}\right)^{2}=4\left(\sqrt{2} A\left(B+B^{t}\right)+\sqrt{2} B A^{t}+C C^{t}\right)_{i j}^{2} .
$$

De maneira similar a estimativa de (D.I.3) temos que

$$
\begin{aligned}
\text { (B.I.3) } & \leq 2\left\|\sqrt{2} A\left(B+B^{t}\right)+\sqrt{2}\left(B+B^{t}\right) A^{t}+C C^{t}\right\|_{F}^{2} \\
& \leq 2\left(\left\|\sqrt{2} A\left(B+B^{t}\right)+\sqrt{2}\left(B+B^{t}\right) A^{t}\right\|_{F}+\left\|C C^{t}\right\|_{F}\right)^{2} \\
& \leq 2\left(2 \sqrt{2}\left\|A\left(B+B^{t}\right)\right\|+\|C\|_{F}^{2}\right)^{2} \\
& \leq 2\left(4\|A\|_{F}\|B\|_{F}+\|C\|_{F}^{2}\right)^{2} \\
& \leq 32\|A\|_{F}^{2}\|B\|_{F}^{2}+16\|A\|_{F}\|B\|_{F}\|C\|_{F}^{2}+2\|C\|_{F}^{4} .
\end{aligned}
$$

Segue que

$$
\text { (B.I) } \leq 56\|A\|_{F}^{2}\|B\|_{F}^{2}+16\|A\|_{F}\|B\|_{F}\|C\|_{F}^{2}+2\|C\|_{F}^{4} \text {. }
$$


Estimando (B.II):

Note que se $\gamma \in\left\{e_{i}\right\}+\left\{e_{i}-e_{j}\right\}$ é tal que existem mais de dois $(\alpha, \beta) \in R_{+} \times R_{+}$tais que $\alpha+\beta=\gamma$ ou se $\gamma$ é soma de duas raízes que formam um ângulo obtuso entre si, devemos ter que

(1) $\gamma=e_{i}+e_{j}-e_{k} \operatorname{com} i<j<k$, ou

(2) $\gamma=e_{i}$.

Então

$$
\text { (B.II) }=\underbrace{\sum_{i<j<k} S_{e_{i}+e_{j}-e_{k}}}_{\text {(B.II.1) }}+\underbrace{\sum_{i} S_{e_{i}}}_{(\text {B.II.2) }} .
$$

Mostraremos que

$$
\text { (B.II.1) } \leq 4\|A\|_{F}^{2}\|C\|_{F}^{2} \quad \text { e } \quad\left(\text { B.II.2) } \leq 8\|A\|_{F}^{2}\|C\|_{F}^{2} .\right.
$$

Estimando (B.II.1):

Se $\alpha, \beta \in R_{+}$são tais que $\alpha+\beta=e_{i}+e_{j}-e_{k} \operatorname{com} i<j<k$ então, a menos de permutações, devemos ter $\alpha=e_{i}-e_{k}$ e $\beta=e_{j}$ ou $\alpha=e_{j}-e_{k}$ e $\beta=e_{i}$. Como em todos esses casos $m(\alpha, \beta)=1$, temos que

$$
S_{e_{i}+e_{j}-e_{k}}=8 a_{i k} c_{j} a_{j k} c_{i} .
$$

Como $a_{i k} c_{j} a_{j k} c_{i}$ é invariante pela permutação de $i$ e $j$ temos que

$$
\begin{aligned}
\sum_{i<j<k} S_{e_{i}+e_{j}-e_{k}} & =8 \sum_{i<j<k} a_{i k} c_{j} a_{j k} c_{i} \\
& \leq 4 \sum_{i, j, k} a_{i k} c_{j} a_{j k} c_{i} \\
& =4 \sum_{k}\left(A^{t} C\right)_{k}^{2} \\
& \leq 4\|A\|_{F}^{2}\|C\|_{F}^{2} .
\end{aligned}
$$

Estimando (B.II.2):

Se $\alpha, \beta \in R_{+}$são tais que $\alpha+\beta=e_{i}$ então, a menos de permutações, devemos ter $\alpha=e_{i}-e_{j}$ e $\beta=e_{j} \operatorname{com} i<j \leq n$. Como em todos esses casos $m(\alpha, \beta)=\sqrt{2}$, temos que

$$
S_{e_{i}} \leq\left(2 \sqrt{2} \sum_{j} a_{i j} c_{j}\right)^{2}=8(A C)_{i}^{2} .
$$

Portanto

$$
\sum_{i} S_{e_{i}} \leq 8\|A C\|_{F}^{2} \leq 8\|A\|_{F}^{2}\|C\|_{F}^{2}
$$

Estimando (B.III):

Se $\gamma \in\left\{e_{i}\right\}+\left\{e_{i}+e_{j}\right\}$ é tal que existem mais de dois $(\alpha, \beta) \in R_{+} \times R_{+} \operatorname{com} \alpha+\beta=\gamma$ devemos ter que $\gamma=e_{i}+e_{j}+e_{k} \operatorname{com} i<j<k$. Note que, a menos de permutações, temos que $\alpha+\beta=e_{i}+e_{j}+e_{k}$ apenas se $\alpha=e_{i}+e_{j}$ e $\beta=e_{k}$ ou $\alpha=e_{i}+e_{k}$ e $\beta=e_{j}$ ou $\alpha=e_{j}+e_{k}$ e $\beta=e_{i}$. Como em todos esses casos $m(\alpha, \beta)=1$, temos que

$$
S_{e_{i}+e_{j}+e_{k}}=8\left(b_{i j} c_{k} b_{i k} c_{j}+b_{i j} c_{k} b_{j k} c_{i}+b_{i k} c_{j} b_{j k} c_{i}\right) .
$$

Usando o fato que $b_{i j} c_{k} b_{i k} c_{j}$ é invariante pela permutação de $k$ e $j$ e que $b_{i k} c_{j} b_{j k} c_{i}$ é invariante pela permutação de $i$ e $j$, é fácil mostrar que 
$\sum_{i<j<k} b_{i j} c_{k} b_{i k} c_{j} \leq \frac{1}{2} \sum_{i, j, k} b_{i j} c_{k} b_{i k} c_{j} \leq \frac{1}{2}\|B\|_{F}^{2}\|C\|_{F}^{2} \quad$ e $\quad \sum_{i<j<k} b_{i k} c_{j} b_{j k} c_{i} \leq \frac{1}{2} \sum_{i, j, k} b_{i k} c_{j} b_{j k} c_{i} \leq \frac{1}{2}\|B\|_{F}^{2}\|C\|_{F}^{2}$.

Como $\sum_{i<j<k} b_{i j} c_{k} b_{j k} c_{i} \leq \sum_{i, j, k} b_{i j} c_{k} b_{j k} c_{i} \leq\|B\|_{F}^{2}\|C\|_{F}^{2}$, temos que

$$
\text { (B.III) } \leq 8\left(\frac{1}{2}\|B\|_{F}^{2}\|C\|_{F}^{2}+\|B\|_{F}^{2}\|C\|_{F}^{2}+\frac{1}{2}\|B\|_{F}^{2}\|C\|_{F}^{2}\right) \leq 16\|B\|_{F}^{2}\|C\|_{F}^{2} .
$$

\subsubsection{Estimativas para $\mathfrak{k}$ excepcional}

Sejam $f, r_{\alpha}$ e $m(\alpha, \beta)$ como na Subsecção 2.2.1. Então temos que

$$
\begin{aligned}
\|\mathrm{II}(f \cdot p, f \cdot p)\|^{2} & \leq \sum_{\gamma \in 2 R_{+}}\left(\sum_{\substack{\alpha, \beta \\
\alpha+\beta=\gamma}} m(\alpha, \beta) r_{\alpha} r_{\beta}\right)^{2} \\
& \leq \sum_{\gamma \in 2 R_{+}}\left(\sum_{\substack{\alpha, \beta \\
\alpha+\beta=\gamma}} m(\alpha, \beta)^{2}\right)\left(\sum_{\substack{\alpha, \beta \\
\alpha+\beta=\gamma}} r_{\alpha}^{2} r_{\beta}^{2}\right) \quad \text { (por Cauchy-Schwartz) }
\end{aligned}
$$

Seja

$$
C=\max _{\gamma \in 2 R_{+}} \sum_{\substack{\alpha, \beta \\ \alpha+\beta=\gamma}} m(\alpha, \beta)^{2},
$$

segue que

$$
\begin{aligned}
\|\mathrm{II}(f . p, f . p)\|^{2} & \leq \sum_{\gamma \in 2 R_{+}} C\left(\sum_{\substack{\alpha, \beta \\
\alpha+\beta=\gamma}} r_{\alpha}^{2} r_{\beta}^{2}\right) \\
& =C\left(\sum_{\alpha \in R_{+}} r_{\alpha}^{2}\right)^{2} \\
& =C .
\end{aligned}
$$

Usando o código no Apêndice B para calcular $C$, temos que

(i) $\|\mathrm{II}\|_{\infty} \leq \sqrt{6}$ se $\mathfrak{k}$ for de tipo $G_{2}$;

(ii) $\|\mathrm{II}\|_{\infty} \leq 2 \sqrt{7}$ se $\mathfrak{k}$ for de tipo $F_{4}$;

(iii) $\|\mathrm{II}\|_{\infty} \leq 2 \sqrt{10}$ se $\mathfrak{k}$ for de tipo $E_{6}$;

(iv) $\|\mathrm{II}\|_{\infty} \leq 8$ se $\mathfrak{k}$ for de tipo $E_{7}$;

(v) $\|\mathrm{II}\|_{\infty} \leq 4 \sqrt{7}$ se $\mathfrak{k}$ for de tipo $E_{8}$.

\subsection{Curvatura de órbitas de representações de tipo real}

Seja $W$ uma representação unitária irredutível de tipo real de $K$. Seja $\lambda$ o peso maximal de $W$ e seja $p \in W_{\lambda}$ qualquer. Como no caso onde $\lambda=0$ as órbitas são discretas, vamos assumir que $\lambda \neq 0$. Sejam $\sigma$ a estrutura real de $W, W_{+}$o conjunto de vetores fixados por $\sigma$ e $\pi$ a projeção ortogonal de $W$ em $W_{+}$. Segue da Proposição 1.3.2 que $\pi=\frac{1}{2}(I d+\sigma)$. Então temos que $\pi$ comuta com os elementos de $K$ e ḱ Logo

$$
T_{\pi(p)} K \pi(p)=\mathfrak{k} \cdot \pi(p)=\pi(\mathfrak{k} \cdot p) .
$$


Mostraremos na Subsecção 2.3.1 que:

Lema 2.3.1. Seja $p$ um vetor de peso maximal da representação unitária irredutível de tipo real $W$. Então existe um subespaço real $U \subset \mathfrak{k} p$ tal que $\pi(U)=\pi(\mathfrak{k} . p)$ e $\sigma(U) \perp U$.

Usando esse lema, podemos demonstrar o Teorema 2.1.2:

\section{Demonstração do Teorema 2.1.2}

Sejam $\mathrm{II}_{\pi}=\mathrm{II}_{\pi(p)}$ e $\mathrm{II}=\mathrm{II}_{p}$. Estamos considerando $K \pi(p)$ como subvariedade da esfera $S_{\pi(p)}(W)$. Como $S_{\pi(p)}\left(W_{+}\right)$é totalmente geodésica em $S_{\pi(p)}(W)$, a norma do supremos da segunda forma fundamental de $K \pi(p)$ é a mesma para essas duas escolhas de variedade ambiente. Como $\pi$ projeta $U$ sobrejetivamente em $\mathfrak{k} \pi(p)$, existe $x \in \mathfrak{k}$ tal que $x . p \in U,\|x . \pi(p)\|=1 \mathrm{e}$ $\left\|\mathrm{II}_{\pi}(x . \pi(p), x . \pi(p))\right\|=\left\|\mathrm{II}_{\pi}\right\|_{\infty}$. Como $x . p \in U$, temos $x . p \perp \sigma(x . p)$ e portanto

$$
1=\|x \cdot \pi(p)\|^{2}=\frac{1}{4}\|x \cdot p+\sigma(x \cdot p)\|^{2}=\frac{1}{2}\|x \cdot p\|^{2} .
$$

Seja $\xi=\frac{\mathrm{II}_{\pi}(x \cdot \pi(p), x \cdot \pi(p))}{\left\|\mathrm{II}_{\pi}(x \cdot \pi(p), x \cdot \pi(p))\right\|}$. Vamos mostrar que $\xi \in W_{+} \cap(\mathfrak{k} . p \oplus \mathbb{R} p)^{\perp}$. Como $K \pi(p) \subset W_{+}$e $W_{+}$ é uma subvariedade totalmente geodésica de $W$ temos que a imagem da segunda forma fundamental de $K \pi(p)$ em $\pi(p)$ é tangente a $W_{+}$, logo $\xi \in W_{+}$. Seja $y \in \mathfrak{k}$ qualquer. Usando que $\xi \in W_{+}$, temos

$$
\begin{aligned}
\langle y \cdot p, \xi\rangle_{\mathbb{R}} & =\langle y \cdot p, \pi(\xi)\rangle_{\mathbb{R}} & \\
& =\langle\pi(y \cdot p), \xi\rangle_{\mathbb{R}} & \\
& =\langle y \cdot \pi(p), \xi\rangle_{\mathbb{R}} & (\text { como } \pi \text { é simétrico }) \\
& =0, & (\text { como que } y \in \mathfrak{k}) \\
& & \text { k } \left.. \pi(p))^{\perp}\right)
\end{aligned}
$$

portanto $\xi \in(\mathfrak{k} . p)^{\perp}$. Como $\xi \in \mathbb{R} \pi(p)^{\perp}$, segue que $\xi \in \mathbb{R} p^{\perp}$.

Concluímos a prova com

$$
\begin{aligned}
\left\|\mathrm{II}_{\pi}\right\|_{\infty} & =\left\|\mathrm{II}_{\pi}(x \cdot \pi(p), x \cdot \pi(p))\right\| \\
& =\left\langle\mathrm{II}_{\pi}(x \cdot \pi(p), x \cdot \pi(p)), \xi\right\rangle_{\mathbb{R}} \\
& =\langle x \cdot x \cdot \pi(p), \xi\rangle_{\mathbb{R}} \\
& =\langle x \cdot x \cdot p, \pi(\xi)\rangle_{\mathbb{R}} \\
& =\langle x \cdot x \cdot p, \xi\rangle_{\mathbb{R}} \\
& =\langle\mathrm{II}(x \cdot p, x \cdot p), \xi\rangle_{\mathbb{R}} \\
& \leq\|\mathrm{II}(x \cdot p, x \cdot p)\| \\
& \leq\|\mathrm{II}\|_{\infty}\|x \cdot p\|^{2} \\
& =2\|\mathrm{II}\|_{\infty}
\end{aligned}
$$

(já que $\xi \in W_{+}$) $\left(\operatorname{como} \xi \in(\mathfrak{k} . p \oplus \mathbb{R} p)^{\perp}\right)$

(por $(2.5))$.

\subsubsection{Demonstração do Lema 2.3.1}

Tomaremos $U=U_{1} \oplus U_{2}$ onde $U_{1}$ é o complemento ortogonal de $\mathfrak{k} . p \cap \sigma(\mathfrak{k} . p)$ em k $p$ e $U_{2}$ um subconjunto de k. $p \cap \sigma(\mathfrak{k} . p)$ tal que $\sigma\left(U_{2}\right) \perp U_{2}$. Antes de construir $U_{2}$ e mostrar que $U$ tem as propriedades desejadas vamos mostrar que

$$
\mathfrak{k} . p \cap \sigma(\mathfrak{k} . p)=\bigoplus_{\alpha \in S} \mathfrak{g}_{-\alpha} \cdot p
$$

onde

$$
S=\left\{\alpha \in R_{+}: \exists \beta \in R_{+} \text {tal que } \lambda-\alpha=\beta-\lambda\right\} .
$$

Note que em vários casos (ex. se o comprimento de $\lambda$ for maior que o comprimento da raiz mais longa de $R$ ) o conjunto $S$ será vazio. Nesses casos teremos $U=\mathfrak{k}$.p. 
Demonstração da identidade (2.6)

Primeiramente, note que por (2.1) temos que

$$
\sigma(\mathfrak{k} . p)=\sigma\left(\mathfrak{n}_{-} . p \oplus \mathbb{R} i p\right)=\sigma\left(\mathfrak{n}_{-} . p\right) \oplus \sigma(\mathbb{R} i p) .
$$

Como $\mathfrak{n}_{-} . p, \sigma\left(\mathfrak{n}_{-} . p\right), \mathbb{R} p$ e $\sigma(\mathbb{R} p)$ são invariantes por $\mathfrak{t} \subset \mathfrak{u}(W)$, temos que

$$
\mathfrak{k} . p \cap \sigma(\mathfrak{k} . p)=\left(\mathfrak{n}_{-} . p \cap \sigma\left(\mathfrak{n}_{-} . p\right)\right) \oplus\left(\mathfrak{n}_{-} . p \cap \sigma(\mathbb{R} i p)\right) \oplus\left(\mathbb{R} i p \cap \sigma\left(\mathfrak{n}_{-} . p\right)\right) \oplus(\mathbb{R} i p \cap \sigma(\mathbb{R} i p)) .
$$

Analisaremos os quatro termos da identidade acima separadamente. Primeiramente, note que por (1.6) temos que $\sigma(\mathbb{R} i p) \subset W_{-\lambda}$. Como $\lambda \neq 0$, segue que

$$
\mathbb{R} i p \cap \sigma(\mathbb{R} i p) \subset W_{\lambda} \cap W_{-\lambda}=0 .
$$

Agora, analisemos o terceiro temo de (2.7). Como $\mathbb{R} p$ e os $\sigma\left(\mathfrak{g}_{-\alpha} \cdot p\right)$ são subespaços de espaços de peso temos que

$$
\mathbb{R} i p \cap \sigma\left(\mathfrak{n}_{-} . p\right)=\bigoplus_{\alpha \in R_{+}} \mathbb{R} i p \cap \sigma\left(\mathfrak{g}_{-\alpha} \cdot p\right)
$$

e $\mathbb{R} i p \cap \sigma\left(\mathfrak{g}_{-\alpha} . p\right) \subset W_{\lambda} \cap W_{\alpha-\lambda}$. Assim $\mathbb{R} i p \cap \sigma\left(\mathfrak{g}_{-\alpha} \cdot p\right) \neq 0$ somente se $\lambda=\alpha-\lambda$. Ou seja, $\mathbb{R} i p \cap \sigma\left(\mathfrak{n}_{-} . p\right) \neq 0$ apenas se $\lambda=\frac{\alpha}{2}$ para algum $\alpha \in R_{+}$, o que o resultado a seguir garante que não ocorre.

Proposição 2.3.2. Seja $W$ uma representação unitária irredutivel de $K$ e suponha que o peso maximal de $W$ é $\frac{\alpha}{2}$ onde $\alpha \in R_{+}$. Então $W$ não pode ser de tipo real.

Demonstração. Assuma por contradição que $W$ tem estrutura real $\sigma$. Seja $\mathfrak{s l}_{\alpha}=\mathfrak{g}_{\alpha} \oplus \mathbb{C} h_{\alpha} \oplus \mathfrak{g}_{-\alpha}$, $\mathfrak{s u}_{\alpha}=\mathfrak{k} \cap \mathfrak{s l}_{\alpha}$ e $\mathrm{SU}_{\alpha} \subset K$ a imagem de $\mathfrak{s u}_{\alpha}$ pela aplicação exponencial. Seja $W^{\prime}=W_{\frac{\alpha}{2}} \oplus W_{-\frac{\alpha}{2}}$. Como $\frac{\alpha}{2}$ é peso maximal, temos que $W^{\prime}$ é invariante por $\mathfrak{s u}_{\alpha}$. Então $W^{\prime}$ também é invariante por $\mathrm{SU}_{\alpha}$. Consideraremos $W^{\prime}$ como uma representação unitária de $\mathrm{SU}_{\alpha}$.

Como o peso maximal de $W^{\prime}$ é $\frac{\alpha}{2}$ temos que $W^{\prime}$ é isomorfo à representação padrão de $\mathrm{SU}(2)$ em $\mathbb{C}^{2}$, que é de tipo quaterniônico. Por outro lado, por (1.6), temos que $W^{\prime}$ é invariante por $\sigma$, então a restrição de $\sigma$ a $W^{\prime}$ é uma estrutura real em $W^{\prime}$. Logo $W^{\prime}$ tem estrutural real e estrutura quaterniônica, contradizendo a Proposição 1.3.5.

De maneira análoga temos que $\mathfrak{n}_{-} . p \cap \sigma(\mathbb{R} i p)=0$. Então (2.7) fica:

$$
\mathfrak{k} . p \cap \sigma(\mathfrak{k} . p)=\mathfrak{n}_{-} . p \cap \sigma\left(\mathfrak{n}_{-} . p\right)=\bigoplus_{\alpha, \beta \in R_{+}} \mathfrak{g}_{-\alpha} \cdot p \cap \sigma\left(\mathfrak{g}_{-\beta} \cdot p\right) .
$$

Como $\mathfrak{g}_{-\alpha} \cdot p \cap \sigma\left(\mathfrak{g}_{-\beta} \cdot p\right) \subset W_{\lambda-\alpha} \cap W_{\beta-\lambda}$ segue que $\mathfrak{g}_{-\alpha} \cdot p \cap \sigma\left(\mathfrak{g}_{-\beta} \cdot p\right) \neq 0$ apenas se $\lambda-\alpha=\beta-\lambda$. A proposição a seguir implica que se $\lambda-\alpha=\beta-\lambda$, então $\mathfrak{g}_{-\alpha} \cdot p \cap \sigma\left(\mathfrak{g}_{-\beta} \cdot p\right)=\mathfrak{g}_{-\alpha} \cdot p$. Logo (2.7) fica igual a (2.6).

Proposição 2.3.3. Sejam $\alpha, \beta \in R_{+}$tais que $\lambda-\alpha=\beta-\lambda$. Então

$$
\mathfrak{g}_{-\alpha} \cdot p=\sigma\left(\mathfrak{g}_{-\beta} \cdot p\right) .
$$

Demonstração. Note que como $\lambda-\alpha=\beta-\lambda$ devemos ter que $\lambda=\frac{\alpha+\beta}{2}$. Consideramos dois casos distintos.

Caso 1: $\alpha \neq \beta$

Note que por (1.6) temos que

$$
W_{\lambda-\alpha}=\sigma\left(W_{\alpha-\lambda}\right)=\sigma\left(W_{\lambda-\beta}\right) .
$$


Como $\mathfrak{g}_{-\alpha} \cdot p \subset W_{\lambda-\alpha}$ e $\mathfrak{g}_{-\beta} \cdot p \subset W_{\lambda-\beta}$, basta mostrar que $\operatorname{dim}_{\mathbb{C}}\left(W_{\lambda-\alpha}\right)=\operatorname{dim}_{\mathbb{C}}\left(\mathfrak{g}_{-\alpha} \cdot p\right)=1 \mathrm{e}$ $\operatorname{dim}_{\mathbb{C}}\left(W_{\lambda-\beta}\right)=\operatorname{dim}_{\mathbb{C}}\left(\mathfrak{g}_{-\beta} \cdot p\right)=1$.

Note que

$$
B\left(\lambda, \alpha^{\vee}\right)=\frac{B\left(\alpha, \alpha^{\vee}\right)+B\left(\beta, \alpha^{\vee}\right)}{2}=1+\frac{B\left(\beta, \alpha^{\vee}\right)}{2} .
$$

Como $B\left(\lambda, \alpha^{\vee}\right)$ é um inteiro não negativo temos que $B\left(\beta, \alpha^{\vee}\right)$ deve ser um número par. Por argumentos análogos temos que $B\left(\alpha, \beta^{\vee}\right)$ também deve ser par. Então pela tabela (A.1) temos que $B(\alpha, \beta)=0 . \operatorname{Logo} B\left(\lambda, \alpha^{\vee}\right)=B\left(\lambda, \beta^{\vee}\right)=1$, e portanto

$$
\lambda-B\left(\lambda, \alpha^{\vee}\right) \alpha=\lambda-\alpha \quad \text { e } \quad \lambda-B\left(\lambda, \beta^{\vee}\right) \beta=\lambda-\beta .
$$

Ou seja, a ação do elemento do grupo de Weil associado a $\alpha$ (resp. $\beta$ ) leva $\lambda$ a $\lambda-\alpha$ (resp. $\lambda-\beta$ ). Então

$$
\operatorname{dim}_{\mathbb{C}}\left(W_{\lambda-\alpha}\right)=\operatorname{dim}_{\mathbb{C}}\left(W_{\lambda}\right)=1 \quad \text { e } \quad \operatorname{dim}_{\mathbb{C}}\left(W_{\lambda-\beta}\right)=\operatorname{dim}_{\mathbb{C}}\left(W_{\lambda}\right)=1 .
$$

Caso 2: $\alpha=\beta$

Note que neste caso a afirmação é equivalente a dizer que $\mathfrak{g}_{-\alpha} \cdot p$ é invariante por $\sigma$. Seja $\mathfrak{s l}_{\alpha}$ como na prova da Proposição 2.3.2. Seja $W^{\prime} \subset W$ a representação irredutível de $\mathfrak{s l}_{\alpha}$ contendo $W_{\lambda}$. Como o peso maximal de $W^{\prime}$ é $\lambda=\frac{\alpha+\beta}{2}=\alpha$, temos que $W^{\prime}$ é isomorfa à representação adjunta de $\mathfrak{s l}(2, \mathbb{C})$. Logo a decomposição de $W^{\prime}$ em espaços peso é

$$
W^{\prime}=W_{-\lambda} \oplus W_{0}^{\prime} \oplus W_{\lambda}
$$



$$
\mathfrak{g}_{-\alpha} \cdot p=W_{0}^{\prime}=\mathfrak{g}_{\alpha} \cdot \sigma(p) .
$$

Tomando $x_{\alpha}$ como na Proposição 1.4.2, temos que

$$
\mathfrak{g}_{\alpha} \cdot \sigma(p)=\mathbb{C} x_{\alpha} \cdot \sigma(p)=\sigma\left(\mathbb{C} x_{\alpha} \cdot p\right)=\sigma\left(\mathfrak{g}_{-\alpha} \cdot p\right) .
$$

Usando esses dois fatos temos que $\mathfrak{g}_{-\alpha} \cdot p=\mathfrak{g}_{\alpha} \cdot \sigma(p)=\sigma\left(\mathfrak{g}_{-\alpha} \cdot p\right)$.

\section{Construção de $U$}

Seja $U_{1}$ o complemento ortogonal de ḱp $p \cap \sigma(\mathfrak{k} . p)$ em k k e seja

$$
U_{2}=\{\pi(x \cdot p)+i \pi(x . p): x \cdot p \in \mathfrak{k} \cdot p \cap \sigma(\mathfrak{k} \cdot p)\} .
$$

Começamos verificando que $U_{2} \subset \mathfrak{k}$. $p \cap \sigma(\mathfrak{k} . p)$. Por (2.6) temos que k $p \cap \sigma(\mathfrak{k} . p)$ é complexo e, como $\sigma$ é involutiva, $\mathfrak{k} . p \cap \sigma(\mathfrak{k} . p)$ é invariante por $\sigma$. Então de fato $U_{2} \subset \mathfrak{k} \cdot p \cap \sigma(\mathfrak{k} . p)$.

Agora mostraremos que podemos tomar $U=U_{1} \oplus U_{2}$. Primeiramente mostraremos que $\sigma(U) \perp$ $U$, em seguida mostraremos que $\pi(U)=\mathfrak{k} . \pi(p)$.

Proposição 2.3.4. Tomando $U=U_{1} \oplus U_{2}$ para $U_{1}$ e $U_{2}$ como descritos acima, temos que $\sigma(U) \perp U$.

Demonstração. Como $\sigma$ é ortogonal, temos que $\sigma(U)=\sigma\left(U_{1}\right) \oplus \sigma\left(U_{2}\right)$. Então

$$
\sigma(U) \perp U \Longleftrightarrow \sigma\left(U_{1}\right) \perp U_{1}, \sigma\left(U_{1}\right) \perp U_{2}, \sigma\left(U_{2}\right) \perp U_{1} \text { e } \sigma\left(U_{2}\right) \perp U_{2} .
$$

Parte 1: Mostramos que $\sigma\left(U_{1}\right) \perp U_{1}$.

Segue de (2.6) que

$$
U_{1}=\mathbb{R} i p \oplus \bigoplus_{\alpha \in R_{+} \backslash S} \mathfrak{g}_{-\alpha} \cdot p
$$


e portanto

$$
\sigma\left(U_{1}\right)=\sigma(\mathbb{R} i p) \bigoplus_{\alpha \in R_{+} \backslash S} \sigma\left(\mathfrak{g}_{-\alpha} \cdot p\right) .
$$

Repetindo os argumentos usados na prova da identidade (2.6), temos que $\sigma\left(U_{1}\right) \perp U_{1}$.

Parte 2: Mostramos que $\sigma\left(U_{1}\right) \perp U_{2}$ e $\sigma\left(U_{2}\right) \perp U_{1}$.

Como $\sigma$ é ortogonal e involutiva temos que $\sigma\left(U_{1}\right) \perp U_{2} \Longleftrightarrow \sigma\left(U_{2}\right) \perp U_{1}$, portanto basta mostrar que $\sigma\left(U_{2}\right) \perp U_{1}$. Note que como $U_{2} \subset \mathfrak{k} p \cap \sigma(\mathfrak{k} p)$ e $\mathfrak{k} p \cap \sigma(\mathfrak{k} p)$ é invariante por $\sigma$ temos que $\sigma\left(U_{2}\right) \subset \mathfrak{k} p \cap \sigma(\mathfrak{k} p)$. Então como, por definição, $\sigma(\mathfrak{k} p) \cap \mathfrak{k} p \perp U_{1}$ temos que $\sigma\left(U_{2}\right) \perp U_{1}$.

Parte 3: Mostramos que $\sigma\left(U_{2}\right) \perp U_{2}$.

Note que para todo $x \cdot p, y \cdot p \in \mathfrak{k} p \cap \sigma(\mathfrak{k} p)$ temos

$$
\begin{aligned}
\langle\sigma(\pi(x \cdot p)+i \pi(x \cdot p)), \pi(y \cdot p)+i \pi(y \cdot p)\rangle_{\mathbb{R}}= & \langle\pi(x \cdot p)-i \pi(x \cdot p), \pi(y \cdot p)+i \pi(y \cdot p)\rangle_{\mathbb{R}} \\
= & \langle\pi(x \cdot p), \pi(y \cdot p)\rangle_{\mathbb{R}}+\langle\pi(x \cdot p), i \pi(y \cdot p)\rangle_{\mathbb{R}} \\
& -\langle i \pi(x \cdot p), \pi(y \cdot p)\rangle_{\mathbb{R}}-\langle i \pi(x \cdot p), i \pi(y \cdot p)\rangle_{\mathbb{R}} \\
= & 2\langle\pi(x \cdot p), i \pi(y \cdot p)\rangle_{\mathbb{R}} .
\end{aligned}
$$

Como $\sigma$ é antilinear e $\pi(y \cdot p)$ é fixado por $\sigma$, segue que $i \pi(y \cdot p)$ é um autovetor de $\sigma$ com autovalor -1 , portanto $\langle\pi(x \cdot p), i \pi(y \cdot p)\rangle_{\mathbb{R}}=0$.

Agora falta apenas mostrar que $\pi(U)=\pi(\mathfrak{k} . p)$. Dado $x \in \mathfrak{k}$, basta mostrarmos que existem $u_{1} \in U_{1}$ e $u_{2} \in U_{2}$ tais que $\pi(x . p)=\pi\left(u_{1}+u_{2}\right)$. Seja $v$ a projeção ortogonal de $x . p$ em $\mathfrak{k} p \cap \sigma(\mathfrak{k} p)$, tome $u_{1}=x . p-v$ e $u_{2}=\pi(v)+i \pi(v)$. Então $u_{i} \in U_{i}$ para $i=1$, 2. Como $i \pi(v)$ é um autovetor de $\sigma$ com autovalor -1 segue que $\pi(i \pi(v))=0$, e portanto

$$
\pi\left(u_{1}+u_{2}\right)=\pi\left(u_{1}\right)+\pi^{2}(v)+\pi(i \pi(v))=\pi\left(u_{1}\right)+\pi(v)=\pi\left(u_{1}+v\right)=\pi(x . p) .
$$




\section{Apêndice A}

\section{Dados sobre sistemas de raízes reduzidos}

Este apêndice contêm dados sobre sistemas de raízes reduzidos simples que são utilizados no capítulo 2 .

\section{Ângulos entre raízes em um sistemas de raízes reduzido}

Sejam $\alpha, \beta$ raízes de um sistema de raízes reduzidos. Segundo [Hum78], os possíveis valores para $B\left(\alpha, \beta^{\vee}\right), B\left(\beta, \alpha^{\vee}\right), \frac{B(\beta, \beta)}{B(\alpha, \alpha)}$ e para o ângulo $\theta$ entre $\alpha$ e $\beta$ são:

\begin{tabular}{cccc}
\hline$B\left(\alpha, \beta^{\vee}\right)$ & $B\left(\beta, \alpha^{\vee}\right)$ & $\frac{B(\beta, \beta)}{B(\alpha, \alpha)}$ & $\theta$ \\
\hline 0 & 0 & Indeterminado & $\frac{\pi}{2}$ \\
1 & 1 & 1 & $\frac{\pi}{3}$ \\
-1 & -1 & 1 & $\frac{2 \pi}{3}$ \\
1 & 2 & 2 & $\frac{\pi}{4}$ \\
-1 & -2 & 2 & $\frac{3 \pi}{4}$ \\
1 & 3 & 3 & $\frac{\pi}{6}$ \\
-1 & -3 & 3 & $\frac{5 \pi}{6}$ \\
\hline
\end{tabular}

Tabela A.1: Possíveis ângulos e produtos de raízes

\section{Dados para sistemas de raízes reduzidos irredutíveis clássicos}

Apresentamos um sistema de raízes $R$ representado cada um dos tipos $A_{n}, B_{n}, C_{n}$ e $D_{n}$. Escrevemos $L$ para o espaço vetorial gerado por $R$, sempre tomamos $L \subset \mathbb{R}^{m}$ e escrevemos $\left\{e_{1}, \cdots, e_{m}\right\}$ para a base canônica de $\mathbb{R}^{m}$. Fixamos uma polarização em $R$ a listamos as raízes positivas. Apresentamos também uma álgebra de Lie complexa $\mathfrak{g}$ que tem $R$ como sistema de raízes. Os dados mostrados aqui foram tomados de [Kna02].

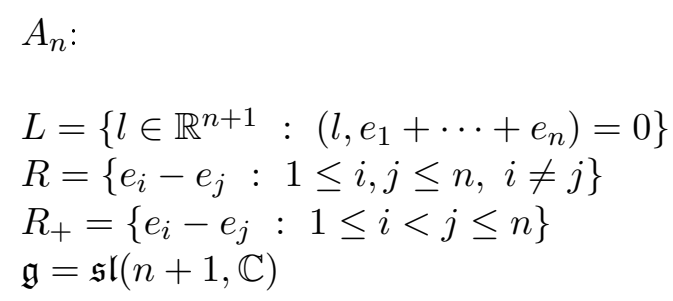


$B_{n}:$

$L=\mathbb{R}^{n}$

$R=\left\{ \pm e_{i} \pm e_{j}: 1 \leq i<j \leq n\right\} \cup\left\{ \pm e_{i}: 1 \leq i \leq n\right\}$

$R_{+}=\left\{e_{i}-e_{j}: 1 \leq i<j \leq n\right\} \cup\left\{e_{i}+e_{j}: 1 \leq i<j \leq n\right\} \cup\left\{e_{i}: 1 \leq i \leq n\right\}$

$\mathfrak{g}=\mathfrak{s o}(2 n+1, \mathbb{C})$

$C_{n}$ :

$L=\mathbb{R}^{n}$

$R=\left\{ \pm e_{i} \pm e_{j}: 1 \leq i<j \leq n\right\} \cup\left\{ \pm 2 e_{i}: 1 \leq i \leq n\right\}$

$R_{+}=\left\{e_{i}-e_{j}: 1 \leq i<j \leq n\right\} \cup\left\{e_{i}+e_{j}: 1 \leq i<j \leq n\right\} \cup\left\{2 e_{i}: 1 \leq i \leq n\right\}$

$\mathfrak{g}=\mathfrak{s p}(2 n, \mathbb{C})$

$D_{n}:$

$L=\mathbb{R}^{n}$

$R=\left\{ \pm e_{i} \pm e_{j}: 1 \leq i<j \leq n\right\}$

$R_{+}=\left\{e_{i}-e_{j}: 1 \leq i<j \leq n\right\} \cup\left\{e_{i}+e_{j}: 1 \leq i<j \leq n\right\}$

$\mathfrak{g}=\mathfrak{s o}(2 n, \mathbb{C})$ 


\section{Apêndice B}

\section{Código}

Neste apêndice apresentamos o código em SageMath usado para demonstrar a Proposição 2.2.5. A função m_sq toma como entrada duas raízes $\alpha$ e $\beta$ e retorna $m(\alpha, \beta)^{2}$ (ver Subsecção 2.2.1) e a função estima_curvatura tem como entrada uma string que especifica o tipo do sistema de raízes (ex. $\mathrm{T}=$ "E6") e retorna $C$ como na Subsecção 2.2.2.




APÊNDICE B 


\section{Referências Bibliográficas}

[AB15] Marcos M. Alexandrino e Renato G. Bettiol. Lie groups and geometric aspects of isometric actions. Springer, Cham, 2015. 1, 7

[BtD95] Theodor Bröcker e Tammo tom Dieck. Representations of compact Lie groups, volume 98 of Graduate Texts in Mathematics. Springer-Verlag, New York, 1995. Translated from the German manuscript, Corrected reprint of the 1985 translation. 8

[DGMS09] William D. Dunbar, Sarah J. Greenwald, Jill McGowan e Catherine Searle. Diameters of 3-sphere quotients. Differential Geom. Appl., 27(2):307-319, 2009. vii

[GL16] Claudio Gorodski e Alexander Lytchak. The curvature of orbit spaces. A aparecer em Geometriae Dedicata, 2016. vii

[Gre00] Sarah J. Greenwald. Diameters of spherical Alexandrov spaces and curvature one orbifolds. Indiana Univ. Math. J., 49(4):1449-1479, 2000. vii

[Hum78] James E. Humphreys. Introduction to Lie algebras and representation theory, volume 9 of Graduate Texts in Mathematics. Springer-Verlag, New York-Berlin, 1978. Second printing, revised. 14, 41

[Kna02] Anthony W. Knapp. Lie groups beyond an introduction, volume 140 of Progress in Mathematics. Birkhäuser Boston, Inc., Boston, MA, second edição, 2002. 41

[McG93] Jill McGowan. The diameter function on the space of space form. Compositio Mathematica, 87(1):79-98, 1993. vii

[MS05] Jill McGowan e Catherine Searle. How tightly can you fold a sphere? Differential Geom. Appl., 22(1):81-104, 2005. vii

[Sak96] Takashi Sakai. Riemannian geometry, volume 149 of Translations of Mathematical Monographs. American Mathematical Society, Providence, RI, 1996. Traduzido da versao original de $1992 \mathrm{em}$ japones pelo autor. 2, 4 